\title{
Quantum Chemical Studies and Pharmacophore Modeling for Designing Novel Keap1 Antagonists that Enhance Nrf2 Mediated Neuroprotection
}

\author{
Srinidhi, ${ }^{1, *}$ \\ 1. Department of Biotechnology, Ramaiah Institute of Technology, MSRIT Post, MSR Nagar, \\ Bengaluru, Karnataka 560054, India. \\ Corresponding author's details \\ Srinidhi \\ Department of Biotechnology, Ramaiah Institute of Technology, MSRIT Post, MSR Nagar, Bengaluru, \\ Karnataka 560054, India \\ Email: msrinidhi2@,gmail.com / srinidh2@,buffalo.edu Ph: +1 - 9282654056.
}

\section{Abstract}

In recent years, the significance of oxidative stress in the pathophysiology of Neurodegenerative/developmental disorders like Attention Deficit Hyperactivity Disorder, Parkinson's and Alzheimer's is being studied at an accelerating pace. Nrf2 activation via Keap1 inhibition is an established strategy for improving the activity of the cellular antioxidant mechanism. In this study, pharmacophore modeling was employed to design efficient Keap1 inhibitors from well-known polypharmacological phytochemicals after extensive structural modifications to improve their pharmacodynamic, pharmacokinetic and drug-likeness qualities $(\mathrm{BBB}>0.9$, HIA $>0.85)$. Density functional theory-based quantum chemical calculations at the B3LYP/6-31G $(d, p)$ level of theory were performed for the geometry optimization of the novel ligands and for computing their electronic properties. Resveratrol-4 was found to be the most desirable candidate with an $\Delta \mathrm{E}=4.24497 \mathrm{eV}$. HOMO and LUMO distribution of the Resveratrol- 4 was found to be very favourable for keap1 binding. Molecular docking studies and comparative interaction analysis also ranked the Resveratrol-4 derivative as the best multi-domain antagonist of the Keapl protein with a binding affinity of $-8 \mathrm{kcal} / \mathrm{mole}$. The following study presents the application of Resveratrol-4 a novel, modified, phytochemical derivative, as an efficient antagonist of the Keap1 protein for enhancing nrf2 mediated neuroprotection from redox insults.

Keywords: Oxidative stress; Attention Deficit Hyperactivity Disorder; Structural Modification; Quantum chemical studies; Nrf2-Keap1

Conflict of interest: None. Number of tables: 5. Number of figures: 11. 


\section{Introduction}

Attention Deficit Hyperactivity Disorder or as described by Dr. Russell Barkley as the "Diabetes of the human brain," is a seriously underrated neurological disorder, if untreated, has lifelong socio-economic repercussions like lower self-regulation for deferred gratification, ergo, higher selfishness, and lower stability in relationships, increased impulsivity, poor executive and cognitive function, poor decision-making ability, lower levels of general happiness and quality of living (Barkley 2014). Etiological studies suggest that Attention Deficit Hyperactivity Disorder is physiologically aggravated due to stress (oxidative and otherwise caused due to lack of physical/mental wellbeing and maintenance) among several other factors.

Oxidative stress is one of the key interdependent factors that cause neurodegeneration. The combinatorial effects of nitrosative and oxidative stress caused by the dysregulation of the neuronal antioxidative mechanism can cause various biochemical aberrations that eventually lead to neuronal cytotoxicity and death. Under excessive oxidative stress from ROS and RNS, the catecholaminergic catabolism proceeds in undesirable secondary oxidative pathways that culminate in the deposition of neurotoxic by-products, by-products which accumulate in the neuroplasm and causes its death and eventual necrosis. Some of the neurotoxins produced from such aberrant pathways are dopamine-o-quinone, dopaminochrome, and 6-hydroxydopamine from dopamine and norepinephrine-o-quinone, norepinephrine semiquinone, and 6-nitronorepinephrine from norepinephrine. Further, The neurotoxic by-products from the secondary oxidative pathways with epinephrine as the substrate resemble norepinephrine by-products (Napolitano et al. 2011; Napolitano et al. 1999a; Napolitano et al. 1999b).

Additionally, other substrates for oxidative stress-induced catabolism are polyunsaturated fatty acids (PUFA) and purines. Unchecked redox imbalance results in the excessive catabolism of cerebral docosahexaenoic acid (Hashimoto et al. 2017; Lauritzen et al. 2016), which terminates in the production of copious amounts of the nontoxic expiratory Attention Deficit Hyperactivity Disorder biomarker, ethane (Ross et al. 2003; Yakubenko and Byzova 2017). Likewise, nucleic acid bases (Adenine and Guanine) under similar conditions produce 8-oxo-7, 8-dihydroguanine (8-oxoG), or 8-oxodeoxyguanosine (8-oxodG), leading to neuronal DNA damage (which could be the etiological basis for the genetic component of Attention Deficit Hyperactivity Disorder) (Chovanová et al. 2006). The accumulation of these toxins results in neuronal cell death and thereby contributes to the pathophysiology and progression of Attention Deficit Hyperactivity Disorder (ND's).

The Nuclear factor erythroid 2-related factor 2 (Nrf2) pathway is the parent cascade that regulates the proteins and enzymes that control neuronal antioxidation. The plethora of products of the Nrf2 associated genes that aid in the neuronal redox homeostasis makes the Nrf2 gene cascade the master regulator of neuronal redox equilibrium. A recent study on Protandim mediated Nrf2 activation in erythrocytes reports a $34 \%$ increase in the amount of superoxide dismutase produced (Nelson et al. 2006). Previous docking studies conducted for nrf2 activation via keap1 inhibition using phytochemicals by Li et al. report that antioxidants are the most efficient class of phytochemicals with the potential to inhibit keapl activity ( $\mathrm{Li}$ et al. 2019). Current research on Nrf2 activation by Bhakkiyalakshmi et al. has championed pterostilbene, which was extensively 
studied in its as-is state for its keap1 inhibition activity (Bhakkiyalakshmi et al. 2016). However, native/unmodified pterostilbene has two reactive methoxy groups, which are highly susceptible to degradation and would eventually pose the problem for which it was used to treat (Increase in ROS and RNS concentration). Therefore, in the present investigation, Resveratrol (a less reactive and naturally occurring derivative of pterostilbene) and other antioxidant phytochemicals were subjected to structural modification and derivatization for increasing their binding efficiency to Keap1 active sites and for degradative self-protection from redox insults.

Structural modification for drug design has been carried out extensively by repurposing existing drugs (Uzzaman and Mahmud 2020), phytochemicals (Gordaliza 2007), peptides (Di 2015), siRNA (Shukla et al. 2010), among other biomolecules as therapeutics. Pharmacophore modeling of pre-existent bio-chemicals has significant advantages over their traditional ab initio counterparts. It requires fewer iterations of computation and the sheer abundance of information available regarding the basal molecule, like its potency, drug-likeness properties, toxicity, pharmacodynamics, and mode of action. Further, quantum chemical studies based on density functional theory are an excellent computational chemistry technique for studying the environment (solvent, cytoplasm, and gas.) specific structure and electrochemical properties of novel drugs. DFT studies provide ample insight into the charge localization, molecular electrostatic potential, reactivity, ionization potential, and electron affinity of the ligands. These quantum chemical descriptors are highly beneficial for understanding the ligands' redox chemistry during biomolecular interactions (ligand-receptor) and are also beneficial in comprehending the ligand's behavior in solvated micro/macro environments.

Herein, structurally modified, novel, antioxidant, phytochemical derivatives that induce Nrf2 activation by inhibiting keap1 activity are presented after a comprehensive investigation by quantum chemical techniques and molecular modeling studies that are augmented by drug-likeness screening and pharmacokinetic analysis.

\section{Materials and Methods}

\subsection{Library Generation and Structural Modification}

A ligand library, comprising of four parent phytochemicals known for their therapeutic and, most importantly, antioxidant properties, namely Decussatin (Karsten et al. 2012), Loliolide (Yang et al. 2011), Resveratrol (stilbene derived polyphenolic phytoalexin found in grapes and raisins) (Gülçin 2010) and Scopoletin (Shaw et al. 2003), were selected for further investigation. The structures, physical and chemical parameters of the phytochemicals were obtained from the PubChem database (Kim et al. 2019). Further, ADMET-SAR (Cheng et al. 2012) software tool and the CB-ligand platform (Liu et al. 2014) were employed to predict the blood-brain barrier permeability index of these four phytochemicals. The blood-brain barrier permeability index of the phytochemicals was predicted to be strongly positive by both of these tools. Therefore, these were finalized as the parent phytochemicals for further ligand library generation via structural modification. Structural modifications were performed primarily using ChemDraw Ultra 12.0 (2D) (Cousins 2011). To increase efficacy, antioxidant activity, and binding efficiency to the active sites of Keap1, the hydroxyl functional groups present in the parent molecules were targeted for 
modification via replacement as they can contribute to post catabolic oxidative stress.

Further, the hydroxyl groups were serially replaced with trifluoromethyl (-CF3) groups (Hagmann 2008; Müller et al. 2007; Purser et al. 2008) and amino (-NH2) groups. It is also noteworthy to know in this context that commercially approved selective serotonin reuptake inhibitors (antidepressants), Fluvoxamine (Brand name: Luvox) (Wilde et al. 1993), and Fluoxetine (Brand name: Prozac) (Altamura et al. 1994) contains one trifluoromethyl moieties each. Further, it is widely known that; the hydroxyl groups

contribute to excess redox imbalance and oxidative damage compared to nitrogen groups (Hybertson et al. 2011). Hence, trifluoromethyl groups (widely known as bio-isosteres) were used in their place to increase binding efficiency as the fluorine groups would increase the reach of the leads in the keap1 binding pockets (Meanwell 2011). The modified 2D structures were converted into a 3-dimensional format using Chem3D Pro 12.0, and the final structures were saved in .sdf and .pdb file formats. Modifications to the Decussatin molecule was not performed as none of its derivatives passed the blood-brain barrier threshold index as set by the two previously mentioned tools. A total of eleven derivatives were designed with 15 ligands in the final ligand library (Figure 1). These ligands were subjected to further studies.Druglikeness Assessment: ADME

\section{2 druglikeness Assessment: ADME}

The ADMET (Absorption, Distribution, Metabolism, Excretion, and Toxicity) assessment of the prepared library was conducted using AdmetSAR (Cheng et al. 2012) tool. A preliminary screening was conducted based on Lipinski's rule of five (Lipinski 2000). Further, the blood-brain barrier permeability parameter was prioritized as the most stringent rule (BBB $>0.80)$. Additional parameters like Human intestinal absorption, Caco-2 permeability, P-glycoprotein substrate and inhibitor, Subcellular localization index, Renal organic cation transporter, HERG inhibition, AMES toxicity, and carcinogenicity were computed for a pervasive assessment of pharmacokinetic desirability. Ligands with severe outliers were simply discarded from the library, and the ligand derivatives with outliers that could merit minor structural modifications were subjected to the same.

\subsection{Quantum Chemical Calculations}

Quantum chemical calculations of the drug-likeness screened library were performed using the General Atomic and Molecular Electronic Structure System (GAMESS-US) software (Gordon and Schmidt 2005). The Avogadro molecule editor and visualizer software (Hanwell et al. 2012) was used to generate input simulation files for the GAMESS program. MacMolPlt software (Bode and Gordon 1998) was utilized for visualizing the output (.log) files. Initial ligand optimization was performed using the Avogadro software. The system's $\mathrm{pH}$ was adjusted to that of cerebrospinal fluid, i.e., 7.2 (Albrecht et al. 2020). The Merck molecular force field 94s (MMFF94s) was set up for primary optimization of the ligand geometry with water as the solvent system. Secondary 
optimization using GAMESS was performed using the unrestricted density functional theory with Becke's (B) three-parameter hybrid model (Becke 1988) along with the Lee, Yang, and Parr's (LYP) non-local correlation functional (B3LYP) (Lee et al. 1988) along with Pople 6-31G (d, p) as the basis set for the geometry optimization and electronic property calculation of all ligands. The energies of the frontier molecular orbitals, highest occupied molecular orbital (HOMO), lowest unoccupied molecular orbital (LUMO), and bandgap were also calculated at the B3LYP/6- 31G (d, p) level of theory. Electronic properties like electronic energy, free energy, dipole moment, molecular electrostatic potential, and bond angles and distances were calculated. The pictorial illustrations of the electronic spin density distributions of the HOMO and LUMO represented ligand structures were generated and color-coded to differentiate the positive and negative electrostatic potentials by using the MacMolPlt visualizer. The Parr and Pearson's interpretation (Lee et al. 1988; Parr and Pearson 1983; Parr and Yang 1995) along with the Koopman's theorem (Koopmans 1934) were utilized for calculating the reactivity parameters viz electron affinity (EA), ionization energy (IA), chemical potential $(\mu)$, hardness $(\eta)$, softness $(\sigma)$, electronegativity $(\chi)$, electrophilicity $(\omega)$, electron-accepting power $\left(\omega^{+}\right)$and electron-donor power $\left(\omega^{-}\right)$of the ligands.

\subsection{Preparation of Ligands}

Ligand preparation was performed using the UCSF Chimera 1.13.1 suite (Pettersen EF, Goddard TD, Huang CC, Couch GS, Greenblatt DM, Meng EC 2004). The ligands were minimized via the structural editor tool with 100 steepest descent steps and ten conjugate gradient steps with a step size of $0.02 \AA$. Missing hydrogens and Gasteiger partial charges were added. AMBER force field 14SB (AMBERFF14SB) was set up during the whole procedure. The structures were saved in pdbqt format for further studies.

\subsection{Receptor Selection and Analysis of Active Site}

The Keap1 protein is thiol-rich (25 cysteine residues), making the protein generally amphoteric and charge dynamic. The Broad complex, Tramtrack, and Bric à brac (BTB) (50-184 amino acid residues) domain of the Keap1 protein is crucial for its homo-dimerization, activity, and structural integrity. The BTB site contains the positively charged, arginine triad residues (R380, R415, and R483, which are crucial for the recognition of the Nrf2 protein) and the histidine residues (H432, H436, H553, H575), which makes the BTB site very basic (Dinkova-Kostova et al. 2002). Further, the Kelch domain (327-611 amino acid residues) in Keap1 (one domain per monomer) is essential for capturing and binding Nrf2 proteins. The DLG and ETGE domains (2382 amino acid residues) in the Neh2 region of the Nrf2 protein are fundamental for it to bind simultaneously to two of the Kelch domain's Keap1 homodimer (Canning et al. 2015; Jnoff et al. 2014). The Kelch domain or Cysteine pocket contains C319, C434, C489, C583, C613, and C624, among other residues, making it hydrophobic and amphoteric (Dinkova-Kostova et al. 2002). Molecular docking analysis was performed with these two domains (BTB and Kelch) as the active sites to investigate the prepared library for dual-site binding efficiency. The structure of the Broad complex, Tramtrack, and Bric à brac (BTB) domain (PDB Code: 5DAD) (Resolution: $2.61 \AA$ ) and the Kelch domain (PDB Code: 4L7B) (Resolution: $2.41 \AA$ ) of the Keap1 protein were obtained from the Protein Data Bank (RCBS) (Berman 2000). Ramachandran Plot analysis was performed to assess the stereochemical suitability and torsional stability of the amino acids of the selected protein receptors via the MolProbity server (Davis et al. 2007) and the PROCHCECK tool 
(Laskowski et al. 1996).

\subsection{Preparation of Receptor}

'Dock Prep' of the UCSF Chimera 1.13.1 suite was employed to prepare the selected receptors for molecular docking simulation. Initially, the co-crystalized, (1S,2R)2[(1S)1[(1,3dioxo2,3dihydro1Hisoindol2yl)methyl]1,2,3,4tetrahydroisoquinolincarbonyl ]cyclohexane1carboxylic acid co-crystallized ligand from the kelch domain of the receptor (4L7B) and the (6aS,7S,10aS)-8-hydroxy4methoxy2,7,10atrimethyll5,6,6a,7,10,10a- hexahydrobenzo [h] quinazoline-9-carbonitrile (TX6201) ligand from the BTB domain of the keap1 receptor (5DAD) were removed. Secondly, the Dunbrack rotamer library was used to add the incomplete side chains of the receptors. Next, missing hydrogens were added for the completed sidechains. Further, Gasteiger partial charges were added, and the AMBER force field 14SB (AMBERFF14SB) was set up for the preparation process. The prepared receptors were saved in pdbqt format and were subject to further analysis.

\subsection{Molecular Docking Simulation}

Molecular docking simulation of the prepared ligands into the prepared receptor was carried out using the widely accepted AutoDock Vina (Trott and Olson 2009) software tool using the PyRx version 0.8 software (Dallakyan and Olson 2015). Rigid docking was performed to investigate the ligand-receptor binding efficiency at the lowest degree of freedom for higher reliability. Water molecules in the system were ignored and were set to not interfere with the simulation in any way. The grid box of the BTB domain (PDB Code: 5DAD) was fixed at X: Y: Z:: -25.4541 : 2.91812: 10.0365 and likewise, the grid box for the Kelch domain (PDB Code: 4L7B) was fixed at $X: Y: Z::-1.50946: 3.16751:-27.1727$ coordinates. The size of the grid box was heuristically adjusted during each simulation to completely incorporate the ligand and the domain that is under purview. Exhaustiveness of the search was set to 10 and the simulation of all ligands including the co-crystalized ligands were conducted. The binding energies $(\mathrm{kcal} / \mathrm{mol})$ of the docked conformations with the highest binding energy coupled with the lowest RMSD (upper and lower bound) were identified, and the same conformations were exported to Accelrys discovery studio (Accelrys Software, Inc., San Diego, CA, USA) for interaction analysis. The ligandreceptor interaction analysis was performed, and docking attributes like type of bonds, interacting atoms-residues, the bond length was recorded, and pictorial illustrations were obtained. Additionally, LigPlot (Laskowski and Swindells 2011) program was also used to assess the ligandreceptor interactions for scaffolding the interaction analysis results obtained from the discovery studio by acting as an additional validator. 


\section{Results and Discussion}

\subsection{Druglikeness Screening and Pharmacokinetic Desirability Assessment}

The complete ligand library qualified the Lipinski's five rule, which was the primary parameter considered for drug-likeness screening. Further, the blood-brain barrier permeability was the second parameter that was prioritized for drug-likeness screening. The top five ligands with the highest BBB permeability were, R4 $>$ L5 $>$ L1 $>$ L $>$ L2. Likewise, the top five ligands with the highest human intestinal absorption were R4 $>$ L5 $>$ L $>$ L2 $>$ L4. Further, Caco2 cell line permeability prediction resulted in the following descending order of penetration in ligands, R4 $>$ L5 $>$ S1 $>$ R1 $>$ S2. It is noteworthy to observe that R4, L5, and S1 are the top three different ligands (with three different parent molecules) with the highest pharmacokinetic desirability. The additional pharmacokinetic analysis resulted in desirable scores for these three ligands concerning their toxicity, metabolism, and distribution. From the results obtained from the conducted druglikeness screening and pharmacokinetics analysis, it can be envisaged that the derivatives, R4, S1, and L5, would successfully permeate the systemic circulatory system and also penetrate past the blood-brain barrier in potent quantities. Upon analysis of the bioavailability radar representations of the ligands, it was found that R4 had minor outliers pertaining to its lipophilicity (XLOGP3; $0.7<$ XLOGP3 $<+5$ ), unsaturation (Fraction Csp3; $0.25<($ Fraction Csp3) $<1$ ) and insolubility $(\operatorname{LogS}(\mathrm{ESOL}) ; 0<\operatorname{LogS}(\mathrm{ESOL})<1)$ values. Likewise, S1 had one outlier in its level of unsaturation. Further, the values of L5 was well within the desirable (pink) region of the bioavailability radar. As obtained from the Swiss-ADME software (Daina et al. 2017), the bioavailability radar illustrations of these ligands are represented in (Figure 2). The results for the complete pharmacokinetic (absorption, distribution, metabolism, elimination) analysis for the entire ligand library is tabulated and presented (Table 1).

Figure 2: Bioavailability radar illustrations: (A) Loliolide (L5); (B) Resveratrol derivative-4 (R4); and (C) Scopoletin (S1).

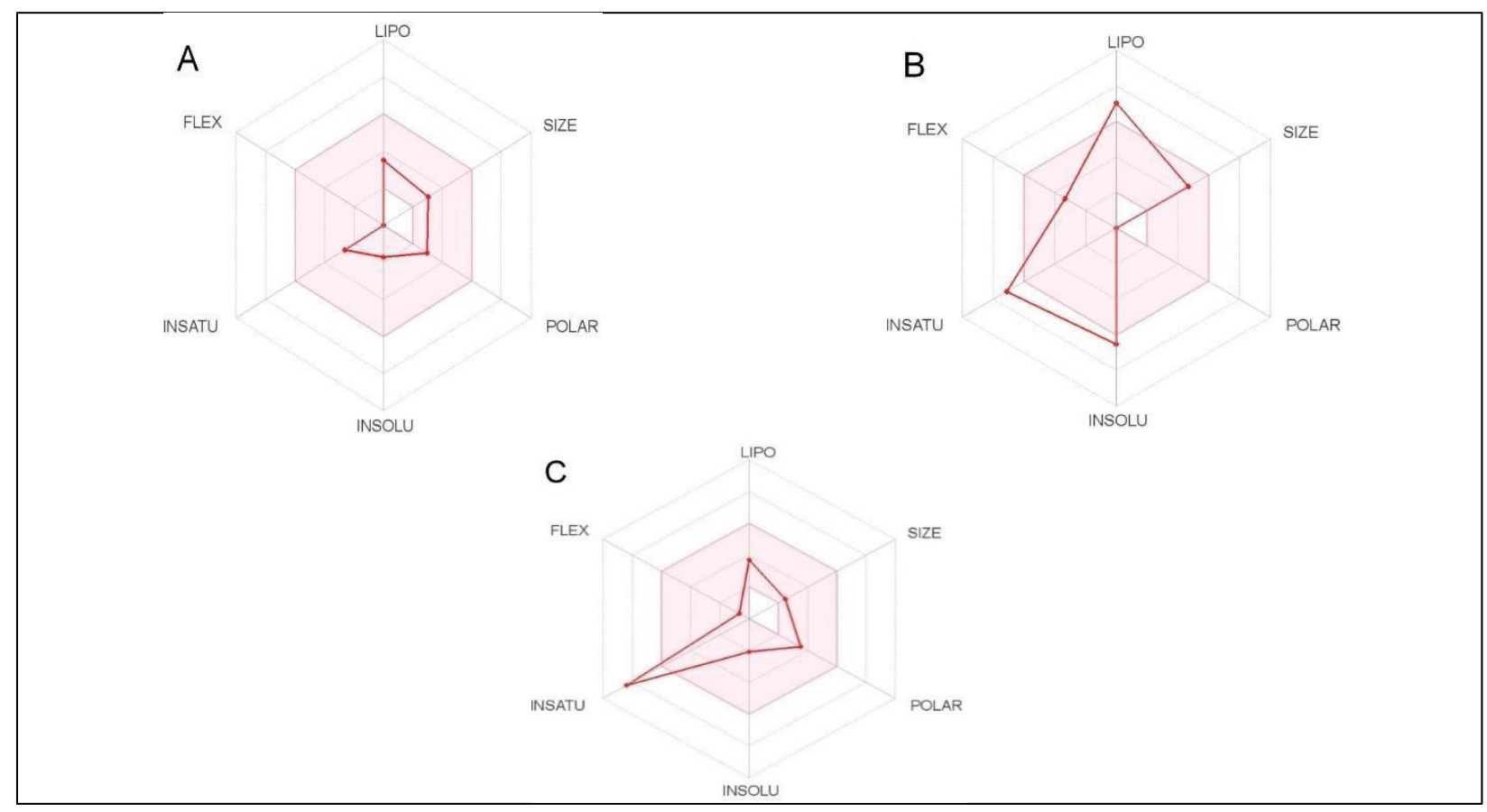


Table 1: Pharmacokinetic analysis of the designed ligand library.

\begin{tabular}{llllllll}
\hline Sl. No Ligands & $\begin{array}{l}\text { Blood- } \\
\text { Brain } \\
\text { Barrier }\end{array}$ & $\begin{array}{l}\text { Human } \\
\text { Intestinal } \\
\text { Absorption }\end{array}$ & $\begin{array}{c}\text { Caco-2 } \\
\text { Permeability }\end{array}$ & $\begin{array}{l}\text { HERG- } \\
\text { Gene } \\
\text { Inhibition }\end{array}$ & $\begin{array}{l}\text { AMES } \\
\text { Toxicity }\end{array}$ & $\begin{array}{l}\text { 'arcinogenicity } \\
\text { (Three-class) }\end{array}$ \\
\hline 1 & Decussatin & 0.5499 & 0.852 & 0.8865 & 0.9463 & 0.8251 & 0.5420 \\
2 & Loliolide & 0.9384 & 1 & 0.5978 & 0.9723 & 0.9498 & 0.5326 \\
3 & L1 & 0.9408 & 0.9937 & 0.5309 & 0.9863 & 0.9331 & 0.5731 \\
4 & L2 & 0.9264 & 1 & 0.5237 & 0.9877 & 0.9118 & 0.591 \\
5 & L3 & 0.8345 & 1 & 0.5223 & 0.9553 & 0.9357 & 0.6692 \\
6 & L4 & 0.8692 & 1 & 0.5065 & 0.9511 & 0.9439 & 0.6637 \\
7 & L5 & 0.9668 & 1 & 0.602 & 0.9591 & 0.924 & 0.5729 \\
8 & Resveratrol & 0.5900 & 0.9952 & 0.8915 & 0.8933 & 0.9462 & 0.6825 \\
9 & R1 & 0.5352 & 0.9831 & 0.5853 & 0.8707 & 0.8629 & 0.8109 \\
10 & R2 & 0.694 & 0.9651 & 0.5549 & 0.9423 & 0.8781 & 0.7352 \\
11 & R3 & 0.7351 & 0.9426 & 0.5599 & 0.9031 & 0.8500 & 0.8179 \\
12 & R4 & 0.9917 & 1 & 0.8014 & 0.9633 & 0.8399 & 0.4747 \\
13 & Scopoletin & 0.7975 & 0.9399 & 0.8944 & 0.9672 & 0.9431 & 0.8059 \\
14 & S1 & 0.7751 & 0.9892 & 0.6018 & 0.9927 & 0.6303 & 0.5913 \\
15 & S2 & 0.5968 & 0.9885 & 0.5737 & 0.9232 & 0.8653 & 0.7800 \\
\hline
\end{tabular}

\subsection{Quantum Chemical Calculations: DFT Studies}

Although the Aufbau principle is used to estimate electrons' allotment into the orbitals of an atom, DFT studies help understand the charge localization and charge migration in a multi-atomic molecular system for varied ionic states. Quantifiable electrochemical properties of the molecule at different ionic states are crucial for understanding its reactivity, behavior, binding affinity (if in a complex), and most certainly, its redox chemistry and antioxidant potential. Geometry optimization of the ligands using DFT studies alleviated the steric hindrance in the molecules, and the most stable coordinates of the ligands were obtained. DFT studies conducted on the constructed ligand library revealed the highest electron affinity for Loliolide-5 at $1.986 \mathrm{eV}$, followed by Resveratrol-4 at $1.8775 \mathrm{eV}$. Scopoletin-5 was placed at ninth place among all ligands. Likewise, the electron-donating power $(\omega-)$ of Loliolide and its derivatives were the highest among all the other ligands (L5 > L > L2 > L4 > L1 > L3). This was followed by Resveratrol-4 with the secondbest electron-donating power $(\omega-)$ at $108.74 \mathrm{eV}(\mathrm{R} 4>\mathrm{R}>\mathrm{R} 1>\mathrm{R} 2>\mathrm{R} 3)$. Scopoletin and its derivatives displayed the lowest transition energy ( $\triangle \mathrm{E}=\mathrm{LUMO}-\mathrm{HOMO})$, indicating the highest bioactivity among the rest $(\mathrm{S} 2>\mathrm{S}>\mathrm{S} 1)$. Meanwhile, resveratrol-4 was ranked at $11^{\text {th }}$ place with $\Delta \mathrm{E}=4.24497 \mathrm{eV}$. Murugavel et al., after experimental and computational studies, have reported a $\Delta \mathrm{E}$ value of $4.6431 \mathrm{eV}$ along with an electron affinity of $1.4438 \mathrm{eV}$ as an excellent energy parameter for their novel human topoisomerase II $\alpha$ inhibitor (Murugavel et al. 2019). Hence, it can be speculated that the Scopoletin derivatives (S, S1-2) and R4 have the highest bioactivity and the potential for being an efficient inhibitor since lower transition energies and higher electron affinities (Top five ligands (including L5, R4, S1) with electron affinities $>1.68710 \mathrm{eV}$ and top 
eleven ligands with $\Delta \mathrm{E}<4.24497 \mathrm{eV}$ ) was observed for the ligands designed in this study. The LUMO was found to be evenly distributed on both the benzene rings of the R4 molecular structure. In contrast, the HOMO orbitals were found to be majorly distributed in and around the central $\mathrm{C} 3=\mathrm{C} 4$ doubly unsaturated linker bond (Figure 3(A)). Likewise, the HOMO orbitals of the S1 derivative were found to be majorly distributed on the heterocyclic (-O) pyran (C10-C12-C11) ring. Whereas, the LUMO orbitals were found to accumulate onto the homo (C7-C6-C14) hexacyclic ring, which is in between the pyran-2-one ring and the adjunct amine (-NH2) and methoxy (-OCH3) functional moieties and to a medium extent over the adjacent heterocyclic pyran ring (Figure 3(B)). The color-coded contour maps of the Molecular electrostatic potentials representing the electronegative and electropositive centers of these ligands were generated and analyzed (Figure 4 (A, B)). The contour maps of the remaining ligands are represented in (Figure (S2)). Finally, the R4 and S1 derivatives of Resveratrol and Scopoletin were found to be the best candidates with the highest potential for both antioxidant and inhibitory binding functions. Hence, these molecules were given more focus for further studies. The quantum reactivity parameters of the ligands are tabulated in (Table (2)). The ligands' electronic properties and energy profile, as computed by GAMESS, are tabulated in (Table (3)).

Figure 3: The color-coded frontier molecular orbitals depicting the Highest occupied molecular orbital (HOMO) and the lowest unoccupied molecular orbital (LUMO): (A) Resveratrol derivative-R4; (B) Scopoletin derivative-S1.

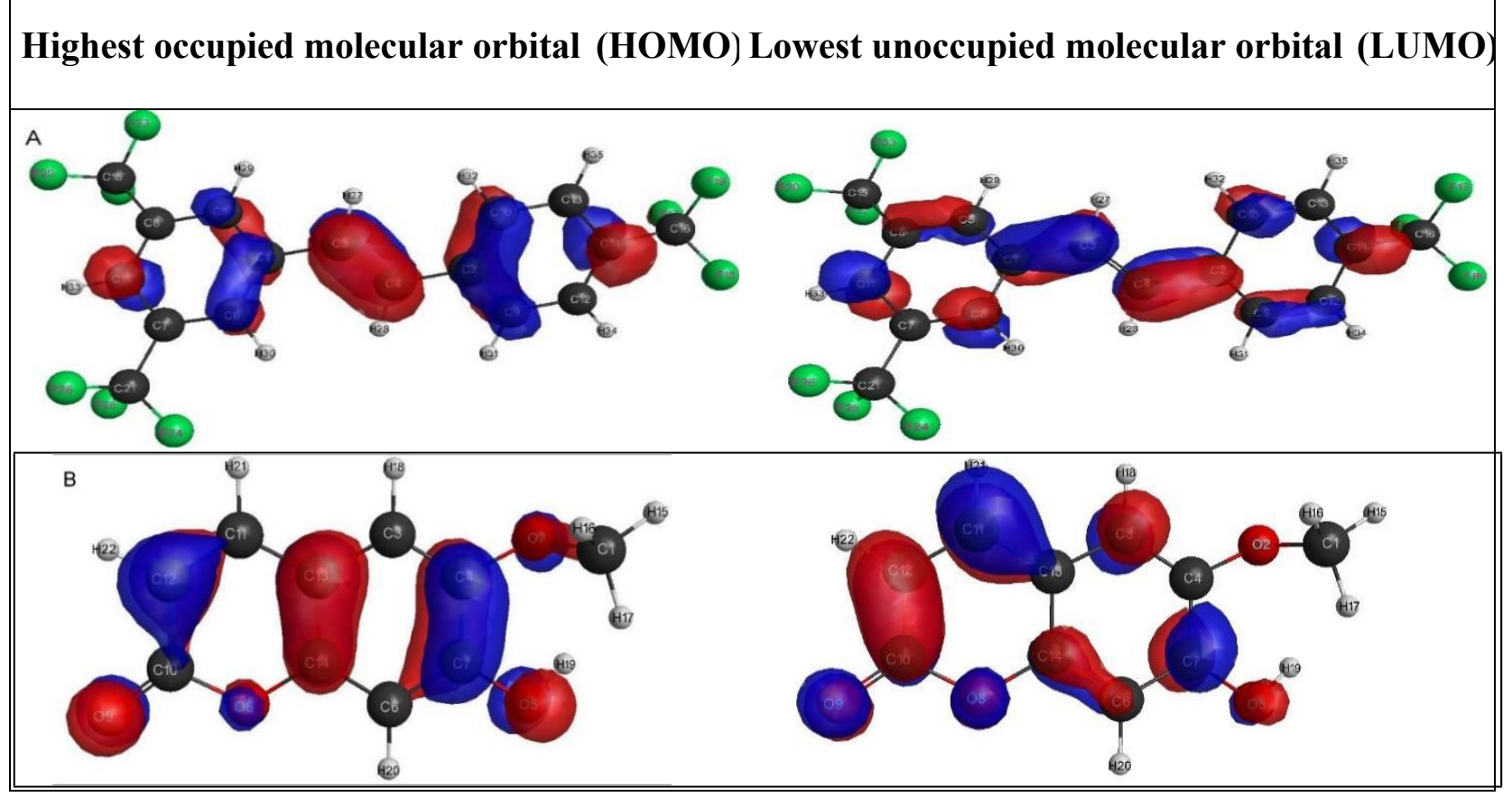


Table 2: The quantum reactivity parameters $(\mathrm{eV})$ computed for the ligand library based on Koopman's theorem and Parr and Pearson's interpretation. (IE: Ionization energy; EA: Electron Affinity; EN: Electronegativity; CP: Chemical Potential; EAP: Electron Accepting Power; EDP: Electron Donating Power).

\begin{tabular}{|c|c|c|c|c|c|c|c|c|c|c|c|c|c|}
\hline \multicolumn{2}{|c|}{ Sl. No ${ }_{\text {Ligand }}$} & \multicolumn{2}{|c|}{ IOMO $(\mathrm{eV}), \mathrm{UMO}(\mathrm{eV})$} & \multicolumn{3}{|c|}{$\Delta E(e V)$ I E $(\mathrm{eV}) \mathrm{E} A(\mathrm{eV}$} & \multirow{2}{*}{$\begin{array}{l}\mathbf{E ~ N} \\
(\chi) \\
(\mathrm{eV})\end{array}$} & \multirow{2}{*}{$\begin{array}{l}\begin{array}{l}\text { C P } \\
(\boldsymbol{\mu}) \\
(\mathrm{eV})\end{array} \\
-3.85\end{array}$} & \multirow{2}{*}{$\begin{array}{l}\begin{array}{l}\text { Iardness } \\
(\mathrm{eV})\end{array} \\
2.03\end{array}$} & \multirow{2}{*}{ 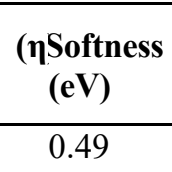 } & \multirow{2}{*}{$\begin{array}{c}\begin{array}{c}\text { Electro } \\
\text { philicity } \\
(\mathbf{e V})\end{array} \\
15.03\end{array}$} & \multirow{2}{*}{$\begin{array}{c}\begin{array}{c}\mathbf{E} A \\
(\omega(\omega+) \\
(\mathbf{e V})\end{array} \\
32.63\end{array}$} & \multirow{2}{*}{$\begin{array}{c}\begin{array}{c}\text { E D P }(\boldsymbol{\omega}- \\
(\mathbf{e V})\end{array} \\
95.92\end{array}$} \\
\hline$\overline{1}$ & Decussatin & -5.88 & -1.82 & 4.05 & 5.88 & 1.82 & & & & & & & \\
\hline 2 & liolide (L) & -7.13 & -1.03 & 6.10 & 7.13 & 1.03 & 4.08 & -4.08 & 3.05 & 0.33 & 25.39 & 39.88 & 191.53 \\
\hline 3 & L1 & -6.48 & -0.71 & 5.77 & 6.48 & 0.71 & 3.59 & -3.59 & 2.88 & 0.35 & 18.61 & 26.66 & 146.19 \\
\hline 4 & $\mathrm{~L} 2$ & -6.61 & -0.63 & 5.99 & 6.61 & 0.63 & 3.62 & -3.62 & 2.99 & 0.33 & 19.60 & 26.97 & 156.67 \\
\hline 5 & L3 & -6.34 & -0.98 & 5.36 & 6.34 & 0.98 & 3.66 & -3.66 & 2.68 & 0.37 & 17.95 & 28.85 & 134.02 \\
\hline 6 & L4 & -6.61 & -0.63 & 5.99 & 6.61 & 0.63 & 3.62 & -3.62 & 2.99 & 0.33 & 19.60 & 26.97 & 156.67 \\
\hline 7 & L5 & -7.81 & -1.99 & 5.82 & 7.81 & 1.99 & 4.90 & -4.90 & 2.91 & 0.34 & 34.93 & 69.00 & 235.09 \\
\hline 8 & veratrol $(\mathrm{R})$ & -5.33 & -1.20 & 4.14 & 5.33 & 1.20 & 3.27 & -3.27 & 2.07 & 0.48 & 11.03 & 20.59 & 76.46 \\
\hline 9 & $\mathrm{R} 1$ & -5.20 & -1.14 & 4.05 & 5.20 & 1.14 & 3.17 & -3.17 & 2.03 & 0.49 & 10.19 & 18.86 & 70.97 \\
\hline 10 & R2 & -5.14 & -1.06 & 4.08 & 5.14 & 1.06 & 3.10 & -3.10 & 2.04 & 0.49 & 9.82 & 17.69 & 69.37 \\
\hline 11 & R3 & -4.98 & -0.98 & 4.00 & 4.98 & 0.98 & 2.98 & -2.98 & 2.00 & 0.50 & 8.88 & 15.68 & 63.35 \\
\hline 12 & R4 & -6.12 & -1.88 & 4.24 & 6.12 & 1.88 & 4.00 & -4.00 & 2.12 & 0.47 & 16.98 & 36.66 & 108.74 \\
\hline 13 & poletin (S) & -6.01 & -1.69 & 4.33 & 6.01 & 1.69 & 3.85 & -3.85 & 2.16 & 0.46 & 16.04 & 33.17 & 105.25 \\
\hline 14 & S1 & -6.01 & -1.69 & 4.33 & 6.01 & 1.69 & 3.85 & -3.85 & 2.16 & 0.46 & 16.04 & 33.17 & 105.25 \\
\hline 15 & $\mathrm{~S} 2$ & -5.47 & -1.50 & 3.97 & 5.47 & 1.50 & 3.48 & -3.48 & 1.99 & 0.50 & 12.05 & 24.63 & 79.60 \\
\hline
\end{tabular}

Figure 4: The color-coded contour maps illustrating the molecular electrostatic potential, the red color depicts electropositive centers, the blue regions represent the neutral potentials, and the green areas represent the electronegative centers: (A) Resveratrol derivative-R4; (B) Scopoletin derivative-S1.

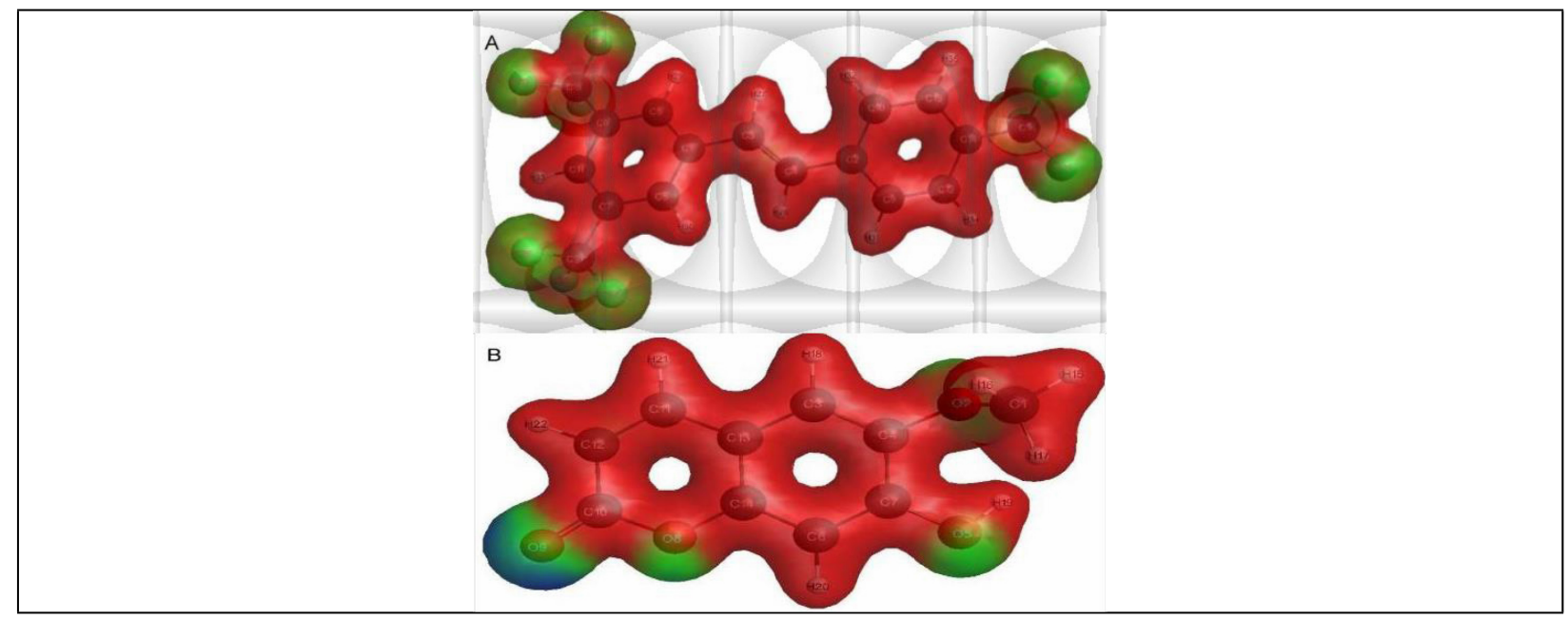




\subsection{Protein Receptor Analysis by Ramachandran Plot}

Ramachandran Plot analysis was performed to assess the selected receptor proteins' structural suitability and torsional stability (PDB: 4L7B / 5DAD). The allowed values of $\psi$ (Psi) against $\varphi$ (Phi) angles for a particular amino acid in a protein was assessed. There are numerous structures of the Keap1 protein deposited into the Protein Data Bank. For the suitability assessment of proteins for further in-silico studies, the Ramachandran plot plays a critical role (Hooft et al. 1997). For the Kelch inhabiting Keap1 protein structure (PDB: 4L7B), 100\% (290/290) of the amino acid residues were reported in the allowed region. Further, 96.2\% (279/290) amino acids were reported to be in the favored area. Likewise, for the BTB domain harboring Keap1 protein structure (PDB: 5DAD), $100 \%(119 / 119)$ of the amino acid residues were reported to be in the allowed region. Further, 95.8\% (114/119) of the amino acids were in the favored area. Hence, these two structures were considered the most suitable, and further studies were conducted. The general case representations of the Ramachandran Plots of the individual proteins are depicted (Figure 5 (A, B)).

Figure 5: General case representations of the Ramachandran plot analysis representations of the selected protein receptor structures depicting the quadrant allocations of the constituent amino acid residues, as obtained from the MolProbity server: (A) BTB domain (PDB: 5DAD); (B) Kelch Domain (PDB: 4L7B).

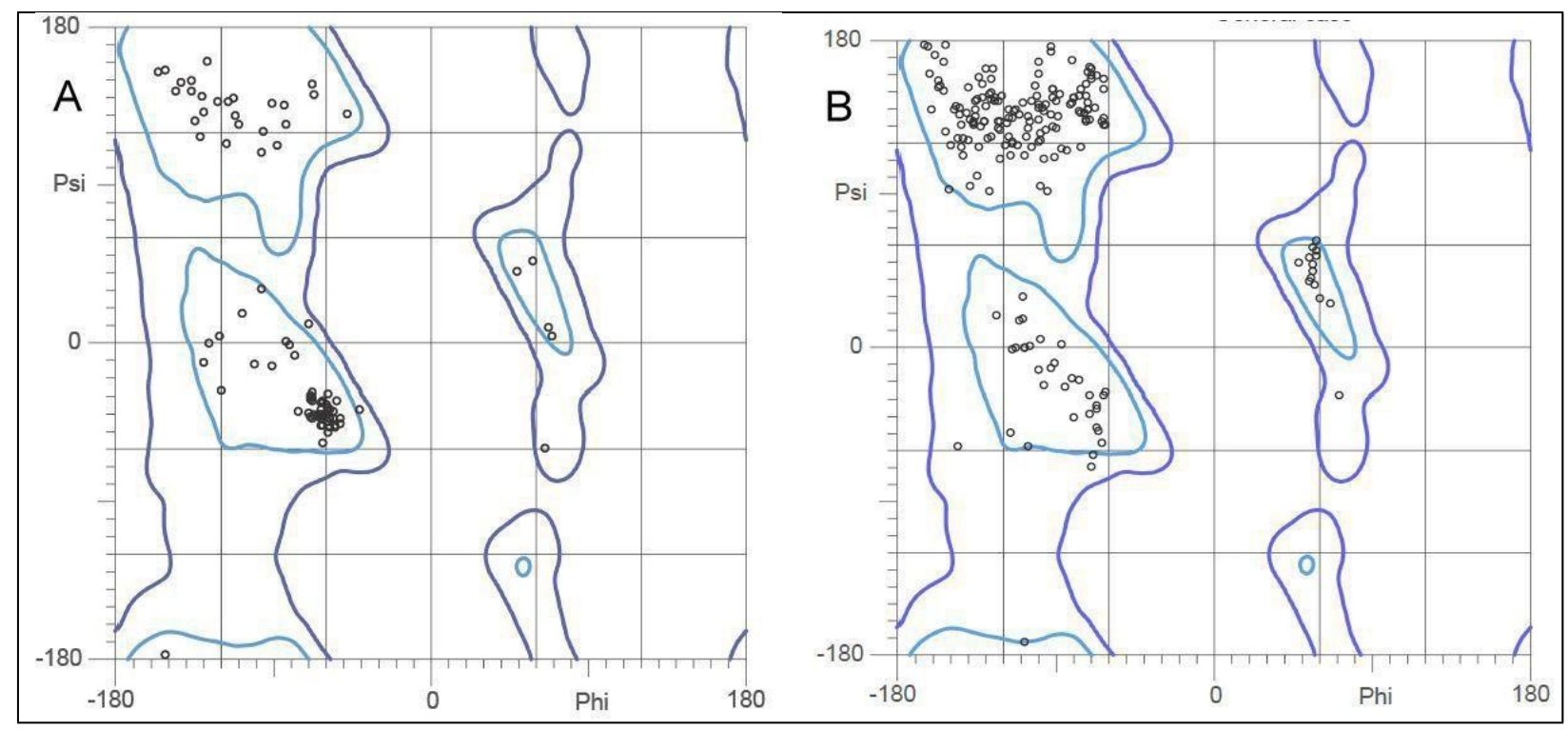




\subsection{Molecular Docking Simulation}

The entire ligand library was subjected to molecular docking and was studied for their binding efficiency with both the BTB domain and Kelch domain of the Keap1 protein. An efficient lead should have excellent binding affinities with both the proteins. Such a ligand would prevent the keap1 protein's homo-dimerization and would inhibit the binding and eventual ubiquitination and proteolytic degradation of the Nrf2 protein. Upon analysis, it was found that the Resveratrol derivative R4 ranked in the first place (lowest root mean square deviation: upper and lower bound) for binding affinities with both the BTB $(-7.3 \mathrm{kcal} / \mathrm{mol})$ and Kelch $(-8 \mathrm{kcal} / \mathrm{mol})$ domains. The top five ligands concerning their binding relationships with the BTB domain were R4 $>$ R $>$ R1 $>$ R2

R3. Unfortunately, the Scopoletin derivatives were ranked at the 7th place in $\mathrm{S}>\mathrm{S} 2>\mathrm{S} 1$, respectively. Likewise, the top five ligands concerning their binding affinities with the Kelch domain were R4 $>$ D $>$ L5 $>$ R $>$ R3 $>$ S1. The therapeutic outcome by inhibitory prevention of the Keap1-Nrf2 complex formation is relatively more significant for enhancing the neuronal antioxidant mechanism than the prevention of the Keap1 homodimerization. Additionally, Pharmacokinetic analysis and quantum chemical studies based on density functional theory have regarded the R4, S1, and L5 derivatives as the best ligands with more promise for exerting a desirable level of therapeutic outcome in the paradigm of this study. Hence, the binding/docking of R4, L5, and S1with the BTB and Kelch domains were studied in depth. The interaction analysis obtained from LigPlot and Accelrys discovery studio, for some ligands, yielded deviant results; this may be because the algorithms employed by this software to predict/identify interactions are different from one another. However, an extensive literature review promotes Accelrys discovery studio to be much more efficient compared to LigPlot. Therefore, the interaction analysis obtained from the discovery studio was given more prominence. Nevertheless, the pictorial representations obtained from LigPlot for the interaction analysis of the ligands that are not under the spotlight of this study with the selected Keap1 and BTB receptors are presented in (Figure S3 (A-O) and Figure S4 (A-O)).

\subsection{Interaction Analysis: BTB Domain}

The Bis(trifluoromethyl)benzene moiety of the R4 derivative was found to be sequestered into the BTB active site, which apparently is sandwiched between the alpha helices (H131-A143 and K150Y162) (Figure 6 (A, B)). Also, The R4 Bis(trifluoromethyl)benzene moiety displayed a Pi donorhydrogen bond and a hydrophobic bond with the Cysteine 151 residue of the BTB active site. Indepth studies conducted on the significance of the cysteine residues in the Keap1 protein by Saito et al. report that the Cysteine 151 residue is indispensable for the keap1 inhibition mediated 'turn-on' mechanism of the cellular antioxidant system as it is one of three significant cellular oxidative stress sensing cysteine residues (Saito et al. 2016). However, Huerta et al. claim that the cysteine 151 binding mediated inhibition itself is not that significant compared to the remaining residues that line the BTB binding pocket for keap1 inhibition mediated Nrf2 accumulation (Huerta et al. 2016). Holding that thought, R4 was found to interact with Valine 155, Histidine 154 and 129, Arginine 135, Lysine 131, Tyrosine 85, and Glutamine 86 (Good agreement with LigPlot 
results (Figure $6(\mathrm{C}))$ ). Further, the fluoride substituted heterocyclic ring adjoining the furan-2-one ring of the L5 derivative was also found to interact with Cysteine 151 residue with a hydrogen bond. L5 also interacted with Lysine 131 and Histidine $(154,129)$ residues of the BTB domain (Figure 7 (A, B)) (Good agreement with LigPlot results (Figure 7 (C))). Finally, S1 was found to interact with Arginine 135, Lysine 131, Histidine 129, Histidine 154, and most importantly, Cysteine 151 with various types of bonds (Figure 8 (A, B)) (Good agreement with LigPlot results (Figure 8 (C))). The complete interaction profile is tabulated in (Table (4)). The remaining pictorial illustrations from PyMol and Discovery studio for the BTB domain is represented in (Figure S5).

Figure 6: The pictorial illustrations for the Resveratrol-4 derivative in the BTB domain (PDB: 5DAD), including the surface view of the top-ranked docked pose (A), interaction analysis from Accelrys discovery studio (B) and the same from LigPlot (C).

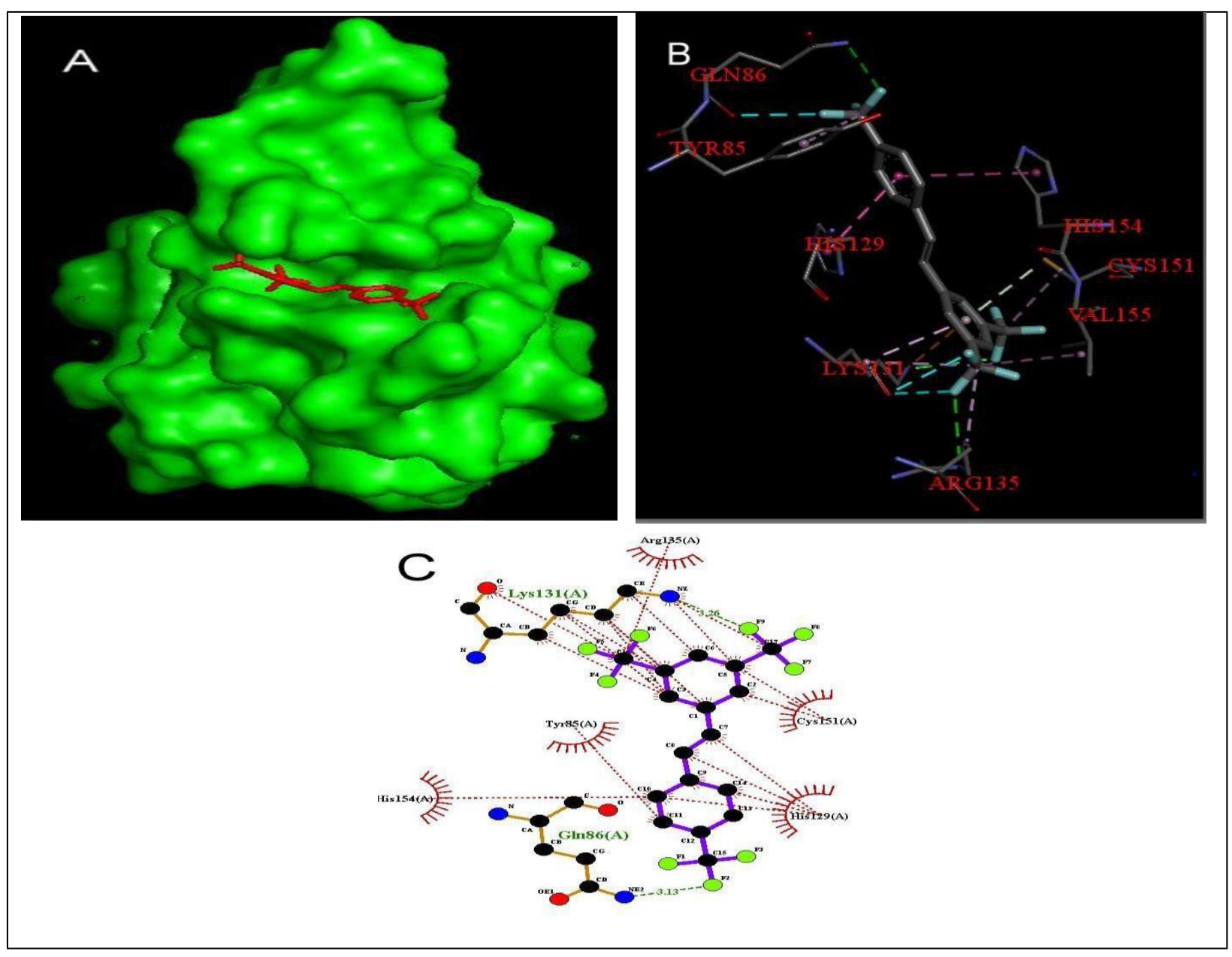


Figure 7: The pictorial illustrations for the Loliolide-5 derivative in the BTB domain (PDB: 5DAD), including the surface view of the top-ranked docked pose (A), interaction analysis from Accelrys discovery studio (B) and the same from LigPlot (C).
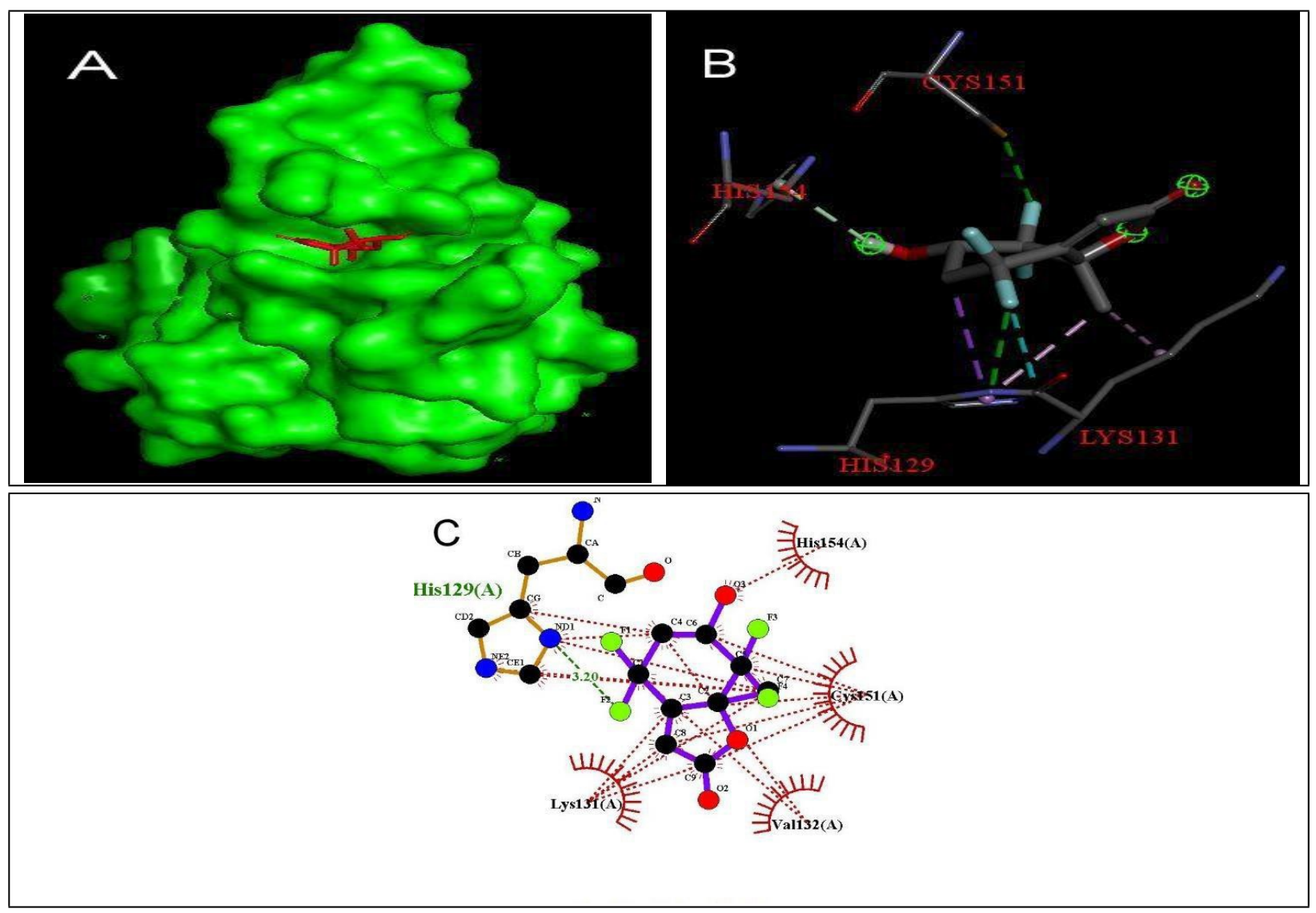

Figure 8: The pictorial illustrations for the Scopoletin-1 derivative in the BTB domain (PDB: 5DAD), including the surface view of the top-ranked docked pose (A), interaction analysis from Accelrys discovery studio (B) and the same from LigPlot (C).
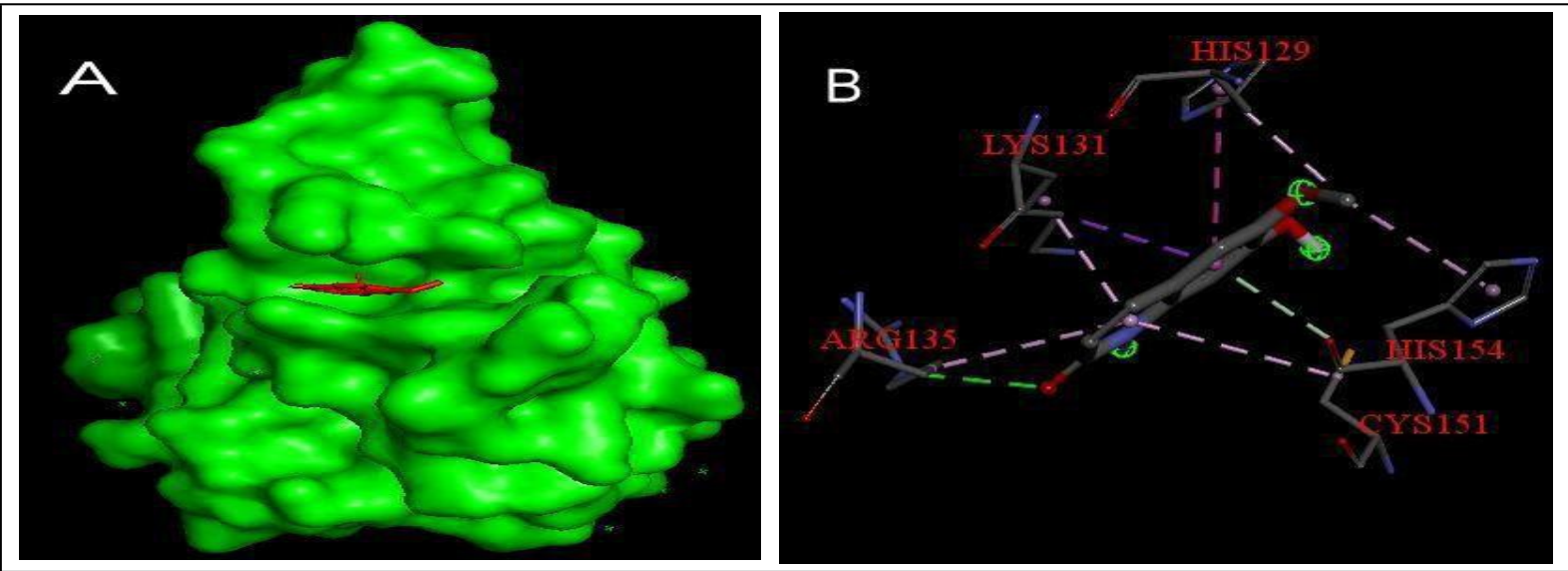


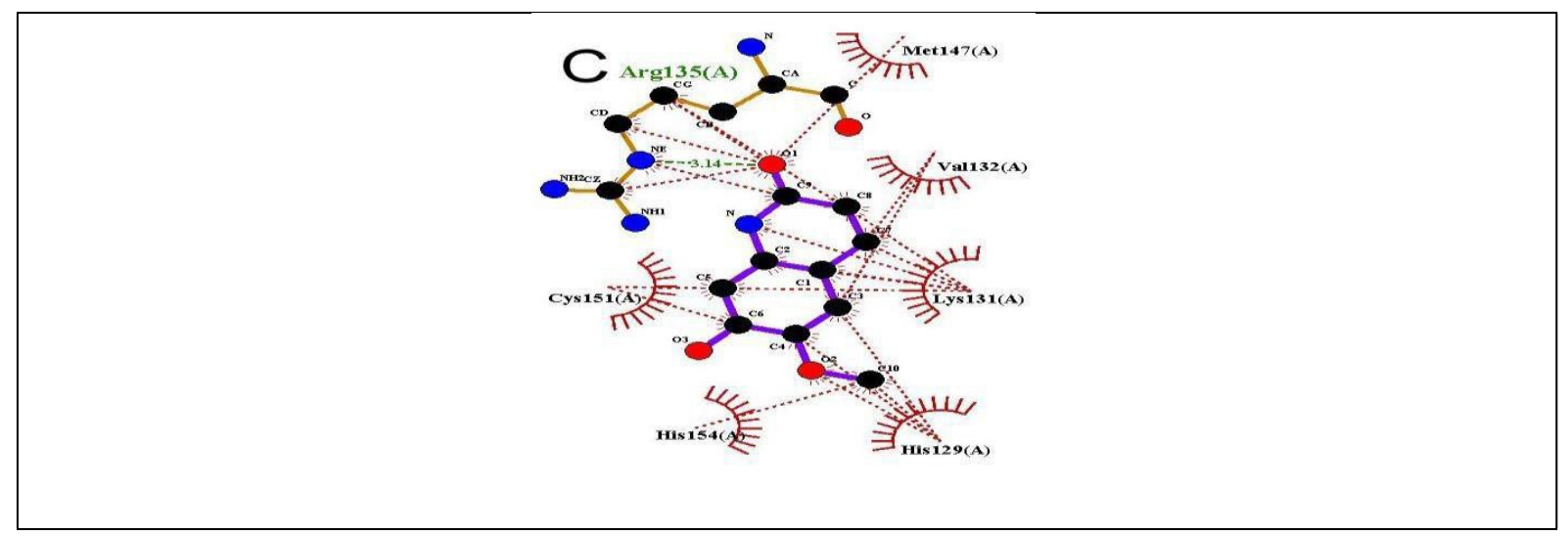

Table 4: Post dock Interaction profile obtained from Accelrys Discovery Studio for the LigandsBTB domain interactions.

\begin{tabular}{llllll}
\hline Name & $\begin{array}{l}\text { Binding } \\
\text { Affinity } \\
\text { (Kcal/Mol) }\end{array}$ & Distance & Bond Category & eraction Typenteracting \\
Residues
\end{tabular}




\begin{tabular}{|c|c|c|c|c|c|}
\hline & & 3.44747 & Hydrophobic & $\mathrm{A}$ & Ays 151 \\
\hline & & 4.77965 & Hydrophobic & A & Val155 \\
\hline & & 5.43301 & Hydrophobic & A & Met147 \\
\hline & & 3.3297 & Hydrophobic & A & Ays 151 \\
\hline & & 4.09246 & Hydrophobic & PA & His 129 \\
\hline \multirow[t]{5}{*}{ Loliolide (L1) } & -4.6 & 3.82918 & Hydrophobic & A & Lys131 \\
\hline & & 3.45466 & Hydrophobic & $\mathrm{A}$ & Cys 151 \\
\hline & & 4.77775 & Hydrophobic & A & Val155 \\
\hline & & 5.43854 & Hydrophobic & $\mathrm{A}$ & Met147 \\
\hline & & 3.3275 & Hydrophobic & A & Cys 151 \\
\hline \multirow[t]{10}{*}{ Loliolide (L2) } & -4.2 & 3.89011 & Hydrophobic & A & Lys 131 \\
\hline & & 4.40328 & Hydrophobic & $\mathrm{A}$ & Cys 151 \\
\hline & & 5.25261 & Hydrophobic & A & Met147 \\
\hline & & 3.30096 & Hydrophobic & A & Cys 151 \\
\hline & & 4.69165 & Hydrophobic & A & Val132 \\
\hline & & 3.48989 & Hydrophobic & A & Cys 151 \\
\hline & & 4.82821 & Hydrophobic & A & Val155 \\
\hline & & 3.82816 & Hydrophobic & A & Lys131 \\
\hline & & 4.77718 & Hydrophobic & A & Val132 \\
\hline & & 4.08718 & Hydrophobic & PA & His 129 \\
\hline \multirow[t]{10}{*}{ Loliolide (L3) } & -4.3 & 3.55522 & Electrostatic & $\mathrm{P}-\mathrm{C}$ & His 129 \\
\hline & & 4.7201 & Electrostatic & $\mathrm{P}-\mathrm{C}$ & His 154 \\
\hline & & 4.89091 & Hydrophobic & A & Val132 \\
\hline & & 3.74529 & Hydrophobic & A & Ays 151 \\
\hline & & 5.06961 & Hydrophobic & A & Val155 \\
\hline & & 3.60548 & Hydrophobic & A & Lys 131 \\
\hline & & 4.38336 & Hydrophobic & A & Val132 \\
\hline & & 4.13823 & Hydrophobic & A & Lys131 \\
\hline & & 3.90624 & Hydrophobic & PA & His 129 \\
\hline & & 4.07472 & Hydrophobic & PA & His 129 \\
\hline \multirow[t]{6}{*}{ Loliolide (L4) } & -3.8 & 4.81785 & Electrostatic & $\mathrm{P}-\mathrm{C}$ & His 129 \\
\hline & & 2.68942 & $\begin{array}{l}\text { Hydrogen Bond; } \\
\text { Electrostatic }\end{array}$ & P-C; PDHB & His 154 \\
\hline & & 4.90609 & Hydrophobic & A & Val132 \\
\hline & & 3.62228 & Hydrophobic & A & Cys 151 \\
\hline & & 5.07086 & Hydrophobic & A & Val155 \\
\hline & & 3.41561 & Hydrophobic & A & Lys 131 \\
\hline
\end{tabular}




\begin{tabular}{|c|c|c|c|c|c|}
\hline & & 4.39384 & Hydrophobic & A & Val132 \\
\hline & & 3.94794 & Hydrophobic & A & Lys131 \\
\hline & & 4.00906 & Hydrophobic & PA & His 129 \\
\hline & & 4.18422 & Hydrophobic & PA & His 129 \\
\hline \multirow[t]{22}{*}{ Resveratrol (R) } & -6.7 & 4.46708 & Electrostatic & $\mathrm{P}-\mathrm{C}$ & Lys $131 z$ \\
\hline & & 3.7492 & Hydrophobic & PPS & His 129 \\
\hline & & 3.87561 & Hydrophobic & $\mathrm{PA}$ & Lys131 \\
\hline & & 5.41155 & Hydrophobic & PA & Arg135 \\
\hline & & 4.57043 & Hydrophobic & PA & Cys 151 \\
\hline & & 4.46708 & Electrostatic & $\mathrm{P}-\mathrm{C}$ & Lys $131 z$ \\
\hline & & 3.7492 & Hydrophobic & PPS & His129 \\
\hline & & 3.87561 & Hydrophobic & PA & Lys131 \\
\hline & & 5.41155 & Hydrophobic & PA & Arg135 \\
\hline & & 4.57043 & Hydrophobic & $\mathrm{PA}$ & Cys 151 \\
\hline & & 3.18767 & Hydrogen Bond & НHB & Arg135 \\
\hline & & 4.46708 & Electrostatic & $\mathrm{P}-\mathrm{C}$ & Lys $131 z$ \\
\hline & & 3.7492 & Hydrophobic & PPS & His129 \\
\hline & & 5.07597 & Hydrophobic & PA & His 129 \\
\hline & & 3.87561 & Hydrophobic & $\mathrm{PA}$ & Lys131 \\
\hline & & 5.41155 & Hydrophobic & PA & Arg135 \\
\hline & & 4.57043 & Hydrophobic & $\mathrm{PA}$ & Cys 151 \\
\hline & & 4.46708 & Electrostatic & $\mathrm{P}-\mathrm{C}$ & Lys $131 z$ \\
\hline & & 3.7492 & Hydrophobic & PPS & His129 \\
\hline & & 3.87561 & Hydrophobic & $\mathrm{PA}$ & Lys131 \\
\hline & & 5.41155 & Hydrophobic & PA & Arg135 \\
\hline & & 4.57043 & Hydrophobic & PA & Cys 151 \\
\hline \multirow[t]{6}{*}{ Resveratrol (R1) } & -6.7 & 2.80703 & Hydrogen Bond & HHB & Arg135 \\
\hline & & 4.43187 & Electrostatic & $\mathrm{P}-\mathrm{C}$ & Lys131 \\
\hline & & 3.7267 & Hydrophobic & PPS & His129 \\
\hline & & 3.94196 & Hydrophobic & $\mathrm{PA}$ & Lys131 \\
\hline & & 5.39084 & Hydrophobic & PA & Arg135 \\
\hline & & 4.58869 & Hydrophobic & $\mathrm{PA}$ & Cys 151 \\
\hline \multirow[t]{7}{*}{ Resveratrol (R2) } & -6.7 & 2.47259 & Hydrogen Bond & HHB & Tyr85 \\
\hline & & 4.61343 & Electrostatic & $\mathrm{P}-\mathrm{C}$ & Lys131 \\
\hline & & 3.8035 & Hydrophobic & PPS & Ais129 \\
\hline & & 4.74585 & Hydrophobic & P-P-TS & Ais 154 \\
\hline & & 3.86137 & Hydrophobic & $\mathrm{PA}$ & Lys131 \\
\hline & & 5.31935 & Hydrophobic & $\mathrm{PA}$ & Arg135 \\
\hline & & 4.59489 & Hydrophobic & PA & Cys 151 \\
\hline
\end{tabular}




\begin{tabular}{|c|c|c|c|c|c|}
\hline \multirow[t]{7}{*}{ lesveratrol (R3) } & -6.4 & 2.91884 & Hydrogen Bond & НHB & Tyr85 \\
\hline & & 2.86593 & Hydrogen Bond & HНB & Ser146 \\
\hline & & 4.55058 & Electrostatic & $\mathrm{P}-\mathrm{C}$ & Lys131 \\
\hline & & 3.81372 & Hydrophobic & PPS & Ais 129 \\
\hline & & 3.7706 & Hydrophobic & PA & Lys131 \\
\hline & & 5.49754 & Hydrophobic & $\mathrm{PA}$ & Arg135 \\
\hline & & 4.5229 & Hydrophobic & PA & Cys 151 \\
\hline \multirow[t]{9}{*}{ Scopoletin (S) } & -5.8 & 3.12433 & Hydrogen Bond & HHB & Arg135 \\
\hline & & 3.88118 & Hydrogen Bond & PDHB & Cys 151 \\
\hline & & 3.74339 & Hydrophobic & P-S & Lys 131 \\
\hline & & 5.0608 & Hydrophobic & PPS & His 129 \\
\hline & & 3.95133 & Hydrophobic & PA & His 129 \\
\hline & & 4.07152 & Hydrophobic & PA & His 154 \\
\hline & & 3.77458 & Hydrophobic & PA & Lys131 \\
\hline & & 5.11458 & Hydrophobic & PA & Arg135 \\
\hline & & 4.77899 & Hydrophobic & PA & Cys151 \\
\hline \multirow[t]{7}{*}{ Scopoletin (S1) } & -5.7 & 3.14201 & Hydrogen Bond & HHB & Arg135 \\
\hline & & 3.87395 & Hydrogen Bond & PDHB & Cys 151 \\
\hline & & 3.77804 & Hydrophobic & $\mathrm{P}-\mathrm{S}$ & Lys 131 \\
\hline & & 5.14951 & Hydrophobic & PA & Arg135 \\
\hline & & 4.74865 & Hydrophobic & PA & Cys151 \\
\hline & & 4.02594 & Hydrophobic & PA & His 154 \\
\hline & & 3.77424 & Hydrophobic & PA & Lys131 \\
\hline \multirow[t]{9}{*}{ Scopoletin (S2) } & -5.8 & 3.14201 & Hydrogen Bond & HHB & Arg135 \\
\hline & & 3.87395 & Hydrogen Bond & PDHB & Ays 151 \\
\hline & & 3.77804 & Hydrophobic & P-S & Lys131d \\
\hline & & 5.0017 & Hydrophobic & PPS & His 129 \\
\hline & & 3.98552 & Hydrophobic & PA & His 129 \\
\hline & & 4.02594 & Hydrophobic & PA & His 154 \\
\hline & & 3.77424 & Hydrophobic & PA & Lys131 \\
\hline & & 5.14951 & Hydrophobic & PA & Arg135 \\
\hline & & 4.74865 & Hydrophobic & PA & Ays 151 \\
\hline \multirow[t]{4}{*}{ iesveratrol (R4) } & -7.3 & 3.12891 & Hydrogen Bond & HHB & $\mathrm{G} \ln 86$ \\
\hline & & 3.25628 & Hydrogen Bond & НHB & Lys 131 \\
\hline & & 3.22821 & Hydrogen Bond & HНB & Arg135 \\
\hline & & 4.01622 & $\begin{array}{l}\text { Hydrogen Bond; } \\
\text { Electrostatic }\end{array}$ & $\mathrm{P}-\mathrm{C}$; PDHB & Lys131 \\
\hline
\end{tabular}




\begin{tabular}{|c|c|c|c|c|c|}
\hline & & 3.85983 & Hydrogen Bond & PDHB & Ays 151 \\
\hline & & 4.19043 & Hydrophobic & PPS & His 129 \\
\hline & & 5.08778 & Hydrophobic & P-P-TS & His 154 \\
\hline & & 4.06628 & Hydrophobic & A & Lys 131 \\
\hline & & 4.10535 & Hydrophobic & A & Arg135 \\
\hline & & 5.14967 & Hydrophobic & A & Val155 \\
\hline & & 4.18138 & Hydrophobic & A & Ays 151 \\
\hline & & 5.3425 & Hydrophobic & PA & Tyr85 \\
\hline & & 3.97664 & Hydrophobic & PA & Lys 131 \\
\hline & & 3.12891 & drogen Bond; Halogen & $\mathrm{CHB} ; \mathrm{X}(\mathrm{F})$ & Gln86 \\
\hline & & 3.25628 & drogen Bond; Halogen & $\mathrm{CHB} ; \mathrm{X}(\mathrm{F})$ & Lys 131 \\
\hline & & 3.22821 & drogen Bond; Halogen & $\mathrm{CHB} ; \mathrm{X}(\mathrm{F})$ & Arg135 \\
\hline Loliolide (L5) & -4.4 & 3.19794 & Hydrogen Bond & HHB & His 129 \\
\hline & & 3.1168 & Hydrogen Bond & НHB & Cys 151 \\
\hline & & 2.65803 & Hydrogen Bond & PDHB & His 154 \\
\hline & & 3.86608 & Hydrophobic & $\mathrm{P}-\mathrm{S}$ & His 129 \\
\hline & & 3.89742 & Hydrophobic & A & Lys 131 \\
\hline & & 3.72478 & Hydrophobic & $\mathrm{PA}$ & His 129 \\
\hline & & 3.19794 & drogen Bond; Halogen & $\mathrm{CHB} ; \mathrm{X}(\mathrm{F})$ & His 129 \\
\hline & & 3.1168 & drogen Bond; Halogen & $\mathrm{CHB} ; \mathrm{X}(\mathrm{F})$ & Cys 151 \\
\hline & & 3.63923 & Halogen & $\mathrm{X}(\mathrm{F})$ & Lys 131 \\
\hline & & 2.65803 & Hydrogen Bond & PDHB & His 154 \\
\hline & & 3.86608 & Hydrophobic & $\mathrm{P}-\mathrm{S}$ & His 129 \\
\hline & & 3.89742 & Hydrophobic & $\mathrm{A}$ & Lys 131 \\
\hline & & 3.72478 & Hydrophobic & PA & His 129 \\
\hline
\end{tabular}

CHB: Carbon Hydrogen Bond; PDHB: Pi-Donor Hydrogen Bond; PPS: Pi-Pi Stacked; A: Alkyl; PA: Pi-Alkyl; HHB: Conventional Hydrogen Bond; P-HB;P-SB: Pi-Donor Hydrogen Bond; PiSulphur; P-C: Pi-Cation; P-C;P-HB: Pi-Cation; Pi-Donor Hydrogen Bond; P-P-TS: Pi-Pi Tshaped; P-S: Pi-Sigma; CHB;X(F): Conventional Hydrogen Bond; Halogen (Fluorine); X(F): Halogen (Fluorine). 


\subsection{Interaction Analysis: Kelch Domain}

The R4 (8kcal/mol) Bis(trifluoromethyl)benzene moiety displayed numerous interactions with the Kelch domain's active site residues. Noteworthy among them were the various types of bonds formed with Arginine 415 and Serine 508 residues deep in the kelch pore of the keap1. Jnoff et al., while depositing the protein structure for the kelch domain of the keap1 protein (PDB: 4L7B), have presented a comparative interaction analysis study between the docking results and their actual co-crystallized (Nrf2-Keap1 complex) inhibitor in the kelch domain of the keap1 protein. In that study, the prominence of the binding induced inhibition facilitated by their novel inhibitor interaction with the Arginine 415 and Serine 508 residues in the kelch pore of the Keap1 protein is elucidated (Jnoff et al. 2014). With the strong interactions displayed by the R4 bis(trifluoromethyl)benzene moiety with the Arginine 415, Serine 508, and the surrounding residues that line the kelch pore, a scenario can be envisaged where the R4 bis(trifluoromethyl)benzene moiety occludes the kelch pore, thereby rendering the Keap1 protein incapable of complex formation with the Nrf2 protein. The reactive yet amphoteric kelch pore would be a suitable microenvironment for the charge stabilized nucleophilic and hydrophobic $\pi$ (ring) systems along with the significantly electronegative trifluoromethyl groups of the R-4 derivative. Meanwhile, the trifluoromethyl-benzene moiety past the double unsaturated hinge region was found to interact with Phenylalanine 577 and Tyrosine 572 residues of the kelch domain at the brim of the pore tunnel (Figure $9(\mathrm{~A}, \mathrm{~B}, \mathrm{C})$ ).

Further, the L5 derivative displayed interactions with kelch pore residues like Serine 602, Glycine 364, Asparagine 414, and 382 (Figure 10 (A, B, C)). The importance of these residues has not been experimentally assessed for their binding mediated keap1 inhibition capability. Hence, no definite conclusions were drawn with regard to their individual/synergistic importance for inhibiting the Keap1 activity. Furthermore, S1 displayed interactions with Kelch residues like Asparagine 414, Arginine 415, Serine 363, Asparagine 382, Tyrosine 334, and Alanine 556. The hydrophobic interactions displayed by Asparagine 414 and Arginine 415 are noteworthy (Figure 11 (A, B, C)). The inter-residue interactions that did not warrant inclusion were excluded while obtaining pictorial illustrations of the interaction analysis. The complete interaction profile is tabulated in (Table (5)). The remaining pictorial illustrations from PyMol and Discovery studio for the Kelch domain is represented in (Figure S6). 
Figure 9: The pictorial illustrations for the Resveratrol-4 derivative in the Kelch domain (PDB: 4L7B), including the surface view of the top-ranked docked pose (A), interaction analysis from Accelrys discovery studio (B) and the same from LigPlot (C).

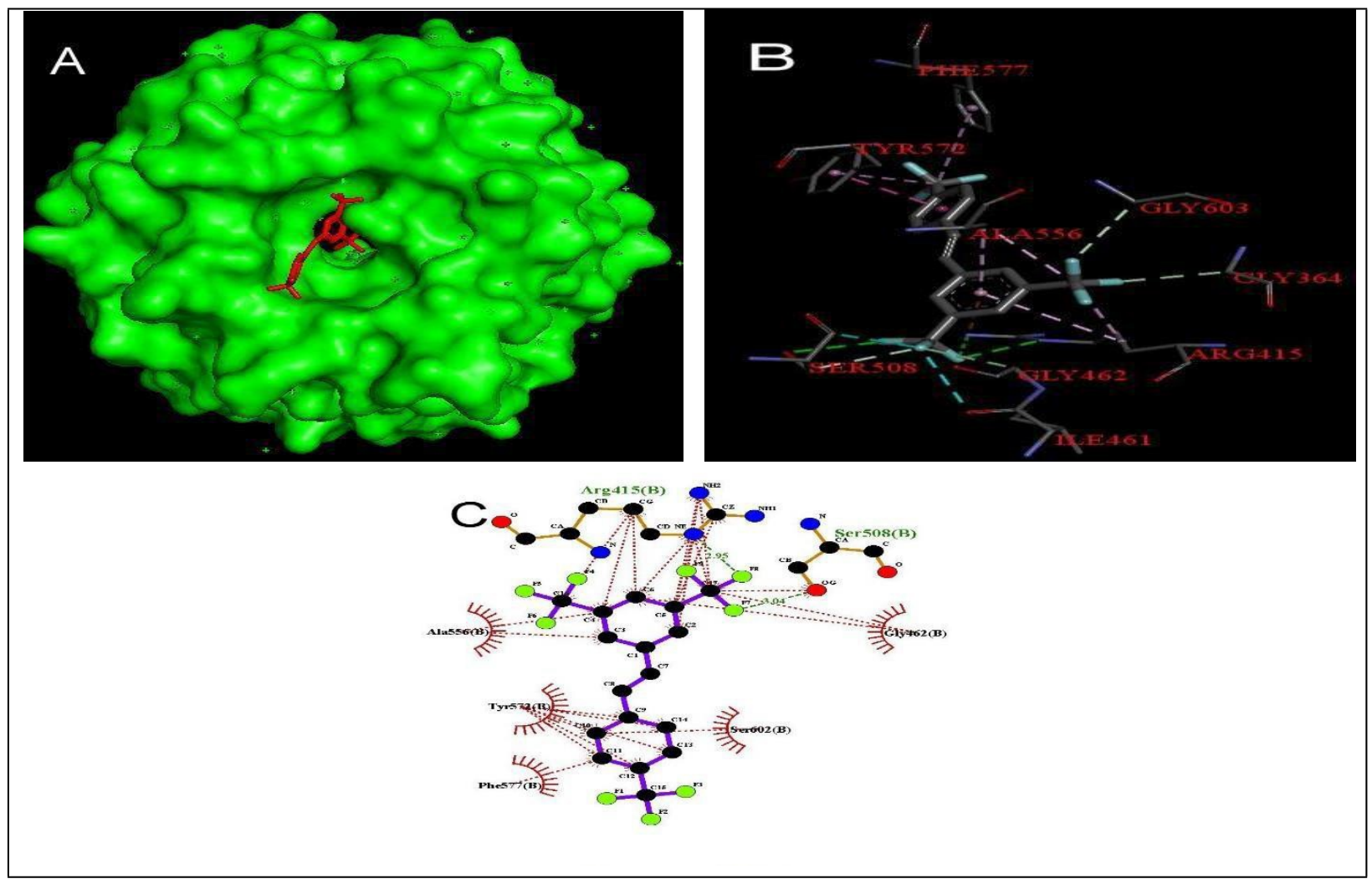

Figure 10: The pictorial illustrations for the Loliolide-5 derivative in the Kelch domain (PDB: 4L7B), including the surface view of the top-ranked docked pose (A), interaction analysis from Accelrys discovery studio (B) and the same from LigPlot (C).

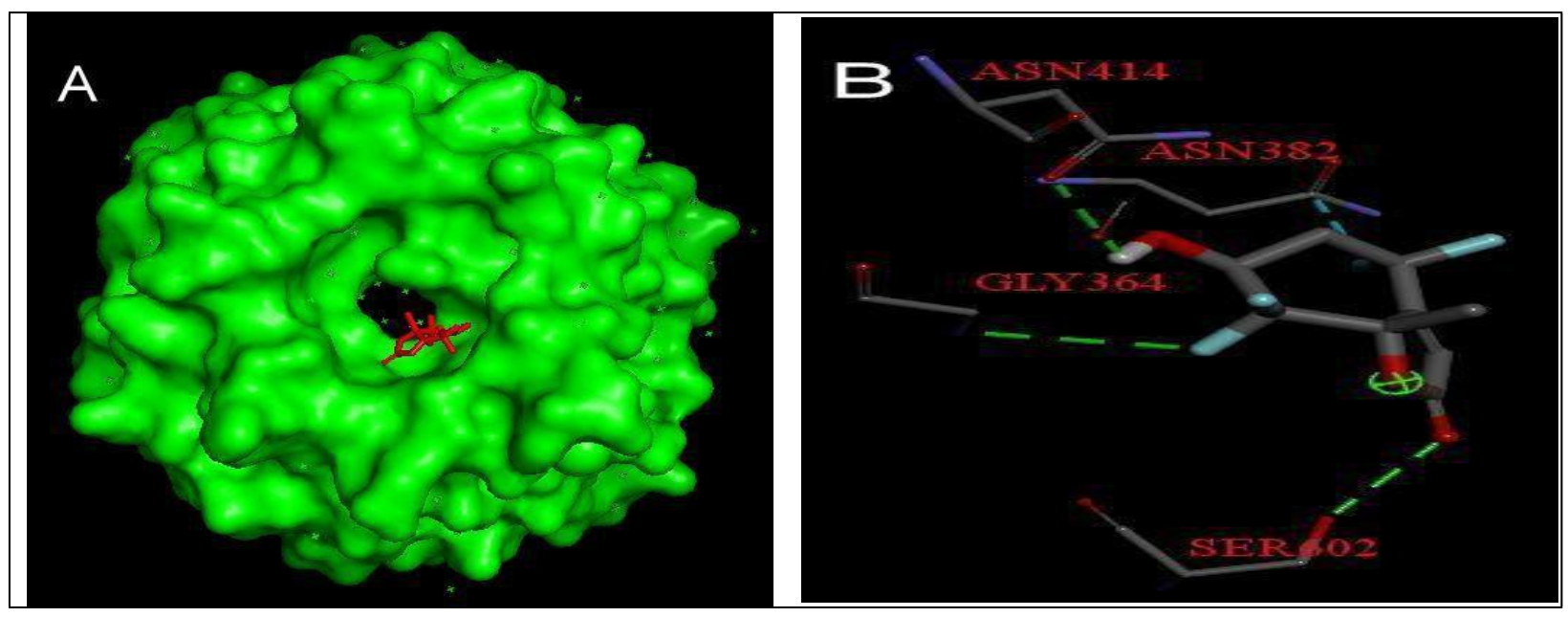




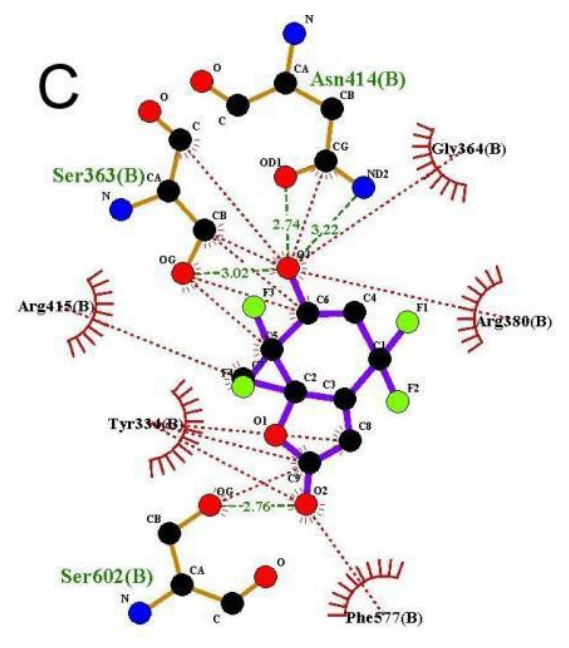

Figure 11: The pictorial illustrations for the Scopoletin-1 derivative in the Kelch domain (PDB: 4L7B), including the surface view of the top-ranked docked pose (A), interaction analysis from Accelrys discovery studio (B) and the same from LigPlot (C).

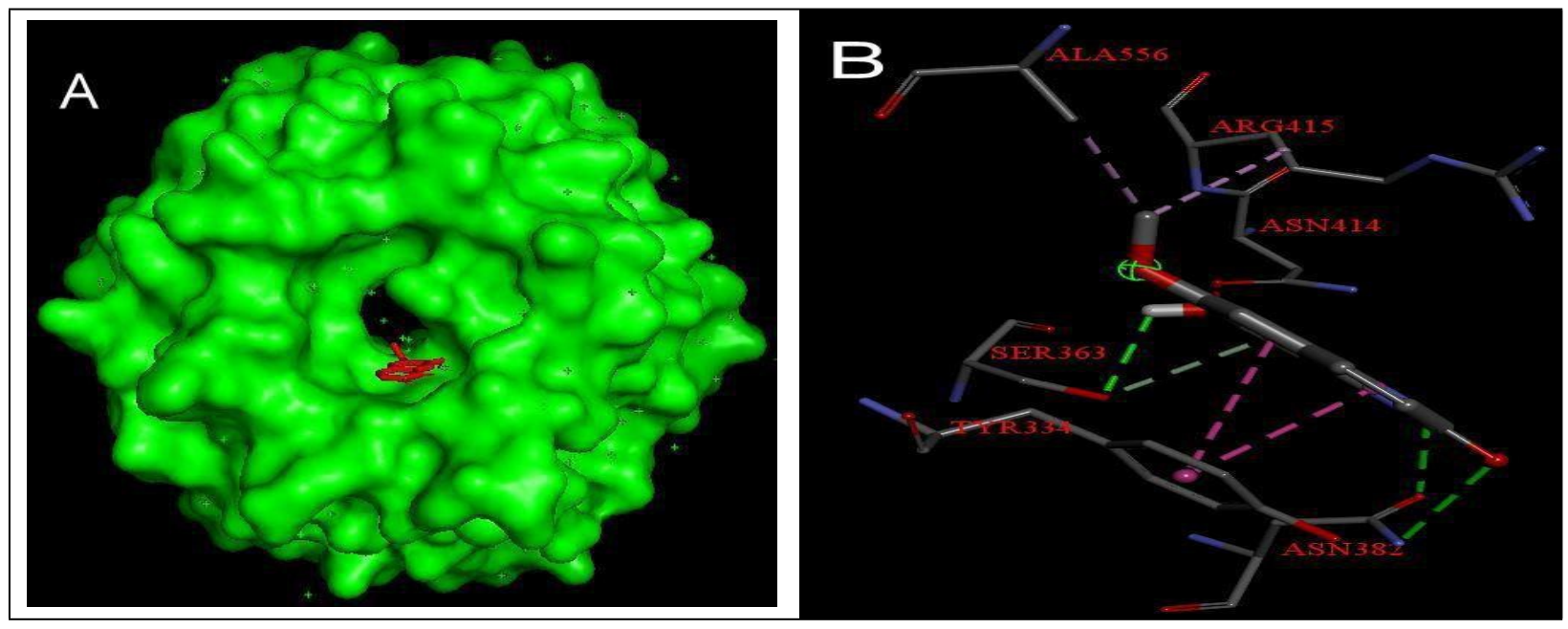




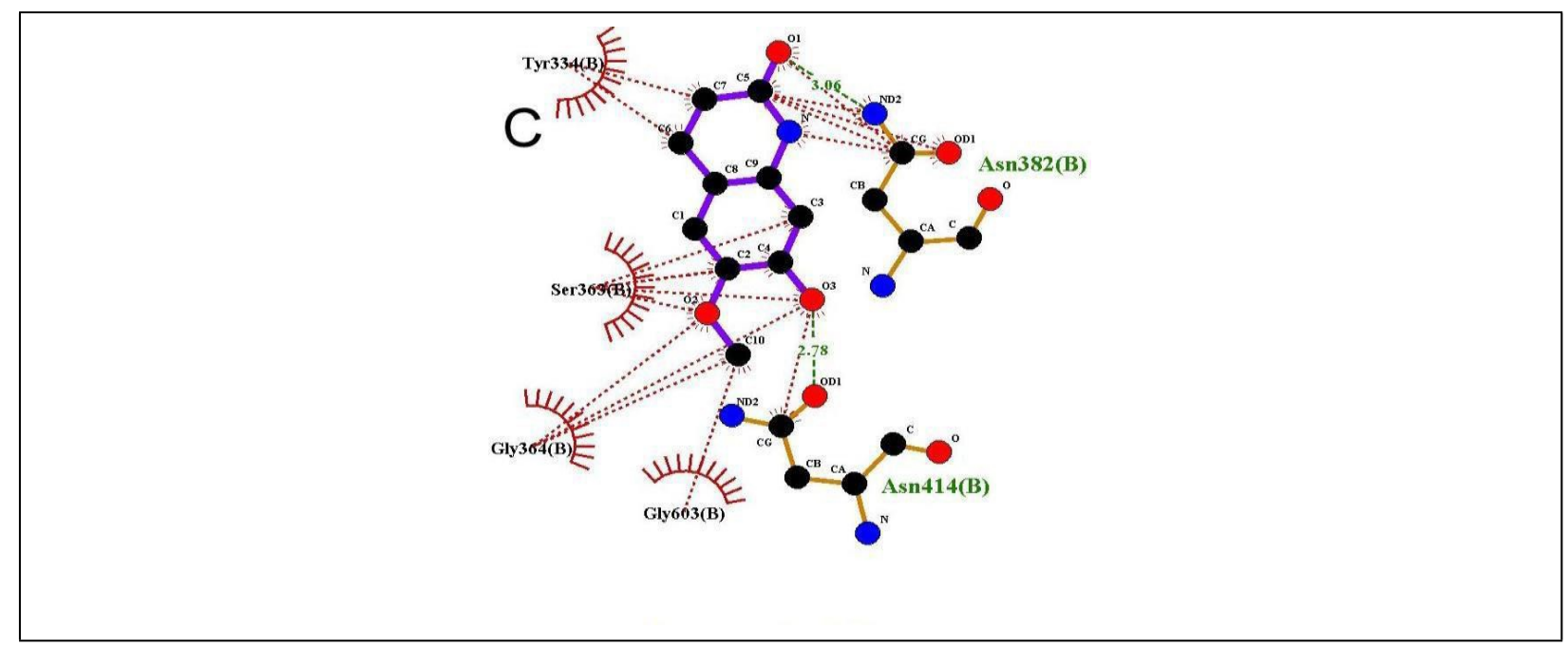

Table 5: Post dock Interaction profile obtained from Accelrys Discovery Studio for the LigandsKelch domain interactions.

\begin{tabular}{|c|c|c|c|c|c|}
\hline Name & $\begin{array}{l}\text { Iding } \\
\text { (Kcal/mol) }\end{array}$ & ${ }^{\text {Affinity }_{\text {Distance }}}$ & Bond Category & eraction type & $\begin{array}{l}\text { nteracting } \\
\text { Residues }\end{array}$ \\
\hline \multirow[t]{9}{*}{ Decussatin } & \multirow[t]{9}{*}{-6.9} & 3.24218 & Hydrogen Bond & HHB & Arg415 \\
\hline & & 3.57364 & Electrostatic & $\mathrm{P}-\mathrm{C}$ & Arg415 \\
\hline & & 3.987 & Hydrophobic & $\mathrm{P}-\mathrm{S}$ & Tyr572 \\
\hline & & 3.95953 & Hydrophobic & $\mathrm{A}$ & Arg415 \\
\hline & & 3.97514 & Hydrophobic & $\mathrm{A}$ & Ala556 \\
\hline & & 5.01244 & Hydrophobic & A & Ile461 \\
\hline & & 4.96178 & Hydrophobic & PA & Ala556 \\
\hline & & 5.09825 & Hydrophobic & PA & Ala556 \\
\hline & & 4.95345 & Hydrophobic & PA & Phe577 \\
\hline \multirow[t]{6}{*}{ Loliolide (L) } & \multirow[t]{6}{*}{-6.2} & 3.0705 & Hydrogen Bond & HНB & Ser363g \\
\hline & & 2.98659 & Hydrogen Bond & HНB & Ser363g \\
\hline & & 5.1654 & Hydrophobic & PA & Tyr334 \\
\hline & & 5.38432 & Hydrophobic & PA & Tyr334 \\
\hline & & 4.56214 & Hydrophobic & PA & Tyr572 \\
\hline & & 5.49526 & Hydrophobic & PA & Phe577 \\
\hline \multirow[t]{6}{*}{ liolide (L1) } & \multirow[t]{6}{*}{-6.3} & 2.86297 & Hydrogen Bond & ННВ & Ser363 \\
\hline & & 2.75771 & Hydrogen Bond & HНB & Ser602 \\
\hline & & 2.5552 & Hydrogen Bond & HHB & Ser363 \\
\hline & & 2.08997 & Hydrogen Bond & HНB & Asn414 \\
\hline & & 5.41112 & Hydrophobic & A & Ala556 \\
\hline & & 3.51456 & Hydrophobic & A & Ala556 \\
\hline
\end{tabular}




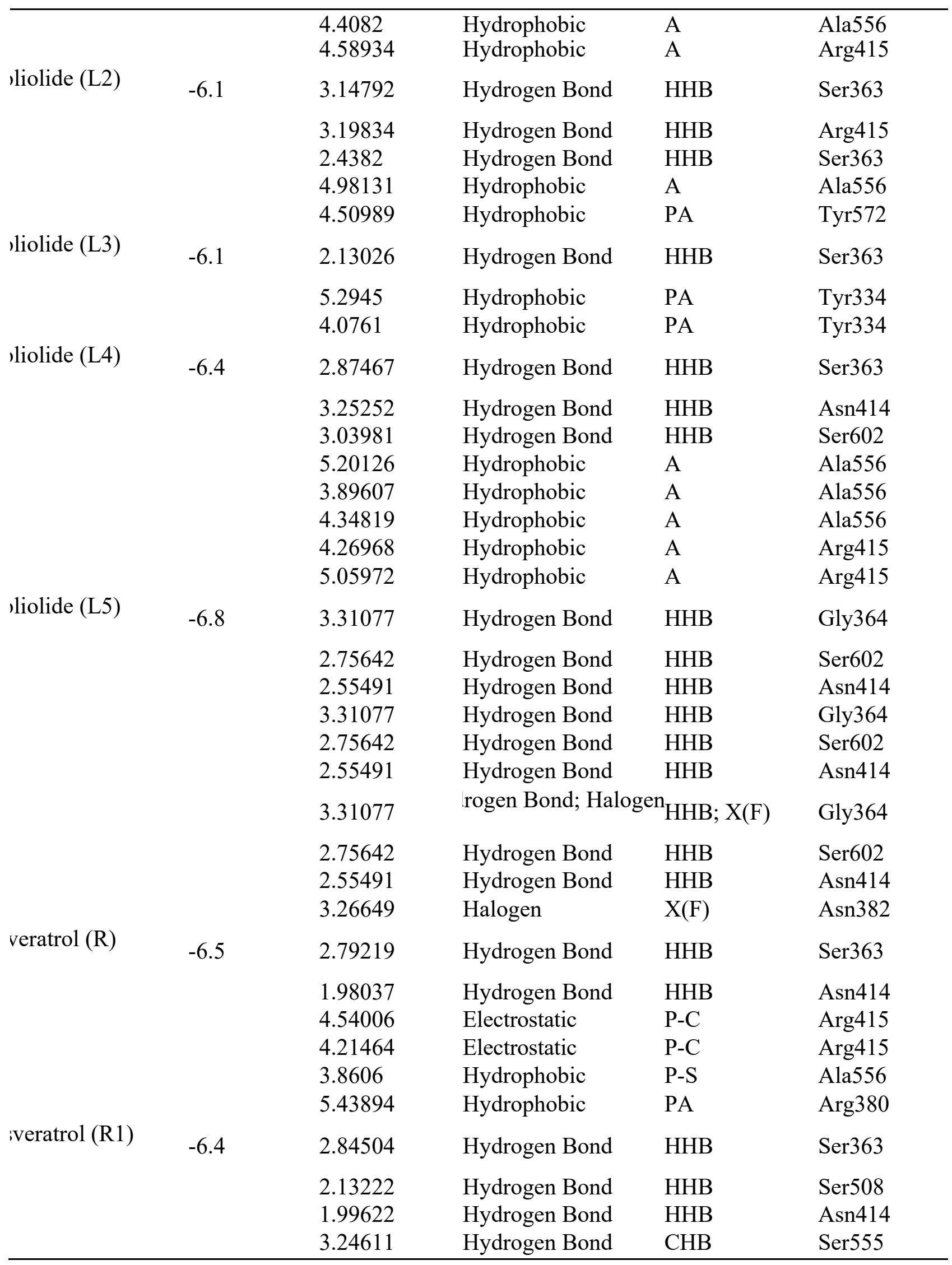




\begin{tabular}{|c|c|c|c|c|c|}
\hline & & 4.66159 & Electrostatic & $\mathrm{P}-\mathrm{C}$ & Arg415 \\
\hline & & 4.34841 & Electrostatic & $\mathrm{P}-\mathrm{C}$ & Arg415 \\
\hline & & 3.72588 & Hydrophobic & $\mathrm{P}-\mathrm{S}$ & Ala556 \\
\hline & & 5.47801 & Hydrophobic & PA & Arg380 \\
\hline iveratrol (R2) & -6.4 & 2.15698 & Hydrogen Bond & HHB & Ser363 \\
\hline & & 2.06752 & Hydrogen Bond & HНB & Ser555 \\
\hline & & 4.07268 & Electrostatic & $\mathrm{P}-\mathrm{C}$ & Arg415 \\
\hline & & 5.63874 & Hydrophobic & P-P-TS & Tyr334 \\
\hline & & 4.69447 & Hydrophobic & $\mathrm{PA}$ & Ala556 \\
\hline iveratrol (R3) & -6.5 & 2.31515 & Hydrogen Bond & НHB & Ser363 \\
\hline & & 2.37749 & Hydrogen Bond & HНB & Ser555 \\
\hline & & 4.15495 & Electrostatic & $\mathrm{P}-\mathrm{C}$ & Arg415 \\
\hline & & 5.75362 & Hydrophobic & P-P-TS & Tyr334 \\
\hline & & 4.6338 & Hydrophobic & PA & Ala556 \\
\hline :veratrol (R4) & -8 & 2.95147 & Hydrogen Bond & HНB & Arg415 \\
\hline & & 3.15531 & Hydrogen Bond & HHB & Arg415 \\
\hline & & 3.03519 & Hydrogen Bond & HHB & Ser508 \\
\hline & & 2.94774 & Hydrogen Bond & $\mathrm{CHB}$ & Gly364 \\
\hline & & 3.07229 & Hydrogen Bond & $\mathrm{CHB}$ & Gly462 \\
\hline & & 3.12396 & Hydrogen Bond & $\mathrm{CHB}$ & Ser508 \\
\hline & & 3.58099 & Hydrogen Bond & $\mathrm{CHB}$ & Gly603 \\
\hline & & 3.94395 & Electrostatic & $\mathrm{P}-\mathrm{C}$ & Arg415 \\
\hline & & 3.69051 & Hydrophobic & PPS & Tyr572 \\
\hline & & 3.92247 & Hydrophobic & $\mathrm{A}$ & Ala556 \\
\hline & & 3.72052 & Hydrophobic & $\mathrm{A}$ & Arg415 \\
\hline & & 4.61022 & Hydrophobic & PA & Tyr572 \\
\hline & & 5.21239 & Hydrophobic & PA & Phe577 \\
\hline & & 4.54073 & Hydrophobic & PA & $\operatorname{Arg} 415$ \\
\hline & & 4.10525 & Hydrophobic & PA & Ala556 \\
\hline poletin $(\mathrm{S})$ & -6.4 & 3.16699 & Hydrogen Bond & НHB & Asn382 \\
\hline & & 2.62422 & Hydrogen Bond & HНB & Ser363 \\
\hline & & 3.55323 & Hydrogen Bond & PDHB & Ser363 \\
\hline & & 5.12251 & Hydrophobic & PPS & Tyr334 \\
\hline & & 4.00382 & Hydrophobic & PPS & Tyr334 \\
\hline & & 4.03552 & Hydrophobic & $\mathrm{A}$ & Ala556 \\
\hline & & 4.61713 & Hydrophobic & A & Arg415 \\
\hline spoletin (S1) & -6.5 & 3.05648 & Hydrogen Bond & HHB & Asn382 \\
\hline & & 2.49493 & Hydrogen Bond & HHB & Asn382 \\
\hline & & 2.7133 & Hydrogen Bond & HHB & Ser363 \\
\hline & & 3.63098 & Hydrogen Bond & PDHB & Ser363 \\
\hline
\end{tabular}




\begin{tabular}{lllll}
\hline & 4.09363 & Hydrophobic & PPS & Tyr334 \\
& 5.10585 & Hydrophobic & PPS & Tyr334 \\
& 3.97733 & Hydrophobic & A & Ala556 \\
& 4.85466 & Hydrophobic & A & Arg415 \\
spoletin (S2) & 3.58029 & Hydrophobic & P-S & Tyr572 \\
& 3.8056 & Hydrophobic & PPS & Tyr334 \\
& 4.47884 & Hydrophobic & PPS & Tyr334 \\
& 4.94473 & Hydrophobic & P-P-TS & Tyr572 \\
& 5.1015 & Hydrophobic & A & Arg415 \\
\hline
\end{tabular}

CHB: Carbon Hydrogen Bond; PDHB: Pi-Donor Hydrogen Bond; PPS: Pi-Pi Stacked; A: Alkyl; PA: Pi-Alkyl; HHB: Conventional Hydrogen Bond; P-HB;P-SB: Pi-Donor Hydrogen Bond; PiSulphur; P-C: Pi-Cation; P-C;P-HB: Pi-Cation; Pi-Donor Hydrogen Bond; P-P-TS: Pi-Pi Tshaped; P-S: Pi-Sigma; CHB; $(\mathrm{F})$ : Conventional Hydrogen Bond; Halogen (Fluorine); X(F): Halogen (Fluorine).

\section{Conclusion}

In this investigation, Resveratrol, Decussatin, Loliolide, and Scopoletin were subjected to in silico derivatization by structural modification via sequential replacement of the parent hydroxyl groups with trifluoromethyl and amine groups resulting in a ligand library. The entire ligand library was subjected to pharmacokinetic analysis (ADME) and was further modified to obtain maximum drug-likeness qualities (Blood-brain-barrier permeability (BBB > 9.0), Human intestinal absorption among others (HIA > 0.85)). Quantum chemical calculations based on density functional theory were performed to optimize the geometries of the constructed library. DFT studies also revealed the ligand's electronic properties (bond lengths, bond angles, HOMO, LUMO, bandgap, ionization potential, and electron affinity) in a water solvent system set to the $\mathrm{pH}$ (7.2) of the human cerebrospinal fluid. R4 (Resveratrol), L5 (Loliolide), and S1 (Scopoletin) derivatives were attributed as superior molecules concerning their potential as an antioxidant/free radical scavenger and also for binding mediated inhibition of the Keap1 protein. Further, molecular docking studies of the ligand library on the Kelch and BTB domains of the Keap1 protein was performed. The post dock comparative interaction analysis suggested remarkable active site residue-ligand bond formations based on which the R4 (Resveratrol) derivative was ranked first (- 8 $\mathrm{Kcal} / \mathrm{mol}$ ) as the best multi-domain inhibitor of the Keap1 protein. Overall, it can be concluded that the performed pharmacokinetic analysis, quantum chemical calculations, and molecular docking studies on the constructed ligand library have produced potent and efficient phytochemical inhibitors (R4, L5, and S1) that can successfully cross the blood-brain barrier and enhance neuronal cytosolic Nrf2 accumulation via Keap1 inhibition. However, the validity of these claims and findings is dependent on in vitro/in vivo experiments, along with the efficiency and reliability of the utilized software.

Further, fluoride toxicity is an issue posed by the novel derivatives presented here (although the rationale for including trifluoromethyl moieties are extensively described previously in this work), the potency of these candidates seems to be high. The conducted investigation and the following results provide strong prima facie evidence that small quantities of these novel candidates possess high therapeutic potential. Additionally, a hybrid prodrug formulation that would 
rectify the problem posed by fluoride toxicity can be a future scope of work. Finally, the R4 molecule can be chemically synthesized by following the catalysis protocols described by (Furuya et al. 2011), and R4 can be fortified along with resveratrol in yogurt, characteristics of which were experimentally studied by (Sonarthi et al. 2018).

\section{Acknowledgements}

To my mother, Uma, with love and gratitude, for instilling in me, her insatiable curiosity and love of learning, among many of her other remarkable attributes.

Dedicated to Shri. S. L. Shivaprasad, Secretary, P.E.S Trust, Mandya, Karnataka, India and to Nadoja Dr. Manu Baligar, President, Kannada Sahitya Parishat, Bangalore, Karnataka, India.

Additionally, the author is grateful for the support from Dr. Bindu S and Mr. T.P. Krishnamurthy sir, Department of Biotechnology, Ramaiah Institute of Technology, Bangalore, Karnataka, India. The author also acknowledges the support from Ms. Harshita Sonarthi (PhD student), Dr. Ramesha K (Professor and Head), Dr. Satish Kumar M H, National Dairy Research Institute, Southern Regional Station, Bangalore, Karnataka, India.

\section{References}

Albrecht B, Voronina E, Schipke C, Peters O, Parr MK, Díaz-Hernández MD, et al. Pursuing Experimental Reproducibility: An Efficient Protocol for the Preparation of Cerebrospinal Fluid Samples for NMR-Based Metabolomics and Analysis of Sample Degradation. Metabolites. MDPI AG; 2020 Jun 16;10(6):251.

Altamura AC, Moro AR, Percudani M. Clinical Pharmacokinetics of Fluoxetine [Internet]. Clin. Pharmacokinet. Springer; 1994. p. 201-14.

Barkley RA. Attention-Deficit Hyperactivity Disorder: A HANDBOOK FOR DIAGNOSIS AND TREATMENT [Internet]. 3rd ed. New York: Guilford; 2014.

Becke AD. Density-functional exchange-energy approximation with correct asymptotic behavior. Phys. Rev. A [Internet]. American Physical Society; 1988 Sep 1;38(6):3098-100.

Berman HM. The Protein Data Bank / Biopython. Presentation. 2000;28(1):235-42.

Bhakkiyalakshmi E, Dineshkumar K, Karthik S, Sireesh D, Hopper W, Paulmurugan R, et al. Pterostilbene-mediated Nrf2 activation: Mechanistic insights on Keap1:Nrf2 interface. Bioorganic Med. Chem. [Internet]. Elsevier Ltd; 2016 Aug;24(16):3378-86.

Bode BM, Gordon MS. MacMolPlt: A graphical user interface for GAMESS. J. Mol. Graph. Model. J Mol Graph Model; 1998 ;16(3):133-8.

Canning P, Sorrell FJ, Bullock AN. Structural basis of Keap1 interactions with Nrf2. 2015;

Cheng F, Li W, Zhou Y, Shen J, Wu Z, Liu G, et al. AdmetSAR: A comprehensive source and free tool for assessment of chemical ADMET properties. J. Chem. Inf. Model. 2012;52(11):3099-105.

Chovanová Z, Muchová J, Sivoňová M, Dvořáková M, Žitňanová I, Waczulíková I, et al. Effect of polyphenolic extract, Pycnogenol ${ }^{\circledR}$, on the level of 8-oxoguanine in children suffering from attention deficit/hyperactivity disorder. Free Radic. Res. 2006;40(9):1003-10. 
Cousins KR. Computer review of ChemDraw ultra 12.0. J. Am. Chem. Soc. 2011. p. 8388.

Dallakyan S, Olson AJ. Small-molecule library screening by docking with PyRx. Methods Mol. Biol. [Internet]. Humana Press Inc.; 2015;1263:243-50.

Davis IW, Leaver-Fay A, Chen VB, Block JN, Kapral GJ, Wang X, et al. MolProbity: Allatom contacts and structure validation for proteins and nucleic acids. Nucleic Acids Res. 2007;35.

Di L. Strategic Approaches to Optimizing Peptide ADME Properties. AAPS J. 2015;17(1):134-43.

Dinkova-Kostova AT, Holtzclaw WD, Cole RN, Itoh K, Wakabayashi N, Katoh Y, et al. Direct evidence that sulfhydryl groups of Keap1 are the sensors regulating induction of phase 2 enzymes that protect against carcinogens and oxidants. Proc. Natl. Acad. Sci. U. S. A. 2002 Sep 3;99(18):11908-13.

Furuya T, Kamlet AS, Ritter T. Catalysis for fluorination and trifluoromethylation. Nature. 2011. p. 470-7.

Gordaliza M. Natural products as leads to anticancer drugs. Clin. Transl. Oncol. 2007;9(12):767-76.

Gordon MS, Schmidt MW. Advances in electronic structure theory: GAMESS a decade later. Theory Appl. Comput. Chem. Elsevier; 2005. p. 1167-89.

Gülçin I. Antioxidant properties of resveratrol: A structure-activity insight. Innov. Food Sci. Emerg. Technol. Elsevier; 2010 Jan 1;11(1):210-8.

Hagmann WK. The many roles for fluorine in medicinal chemistry [Internet]. J. Med. Chem. J Med Chem; 2008. p. 4359-69.

Hanwell MD, Curtis DE, Lonie DC, Vandermeerschd T, Zurek E, Hutchison GR. Avogadro: An advanced semantic chemical editor, visualization, and analysis platform. J. Cheminform. BioMed Central; 2012 Aug 13;4(8):17.

Hashimoto M, Hossain S, Al Mamun A, Matsuzaki K, Arai H. Docosahexaenoic acid: one molecule diverse functions. Crit. Rev. Biotechnol. 2017;37(5):579-97.

Hooft RWW, Sander C, Vriend G. Objectively judging the quality of a protein structure from a Ramachandran plot. CABIOS. 1997.

Huerta C, Jiang X, Trevino I, Bender CF, Ferguson DA, Probst B, et al. Characterization of novel small-molecule NRF2 activators: Structural and biochemical validation of stereospecific KEAP1 binding. Biochim. Biophys. Acta - Gen. Subj. Elsevier B.V.; 2016;1860(11):2537-52.

Hybertson BM, Gao B, Bose SK, McCord JM. Oxidative stress in health and disease: The therapeutic potential of Nrf2 activation. Mol. Aspects Med. [Internet]. 2011 Aug;32(4-6):234-46. 098299711000501

Jnoff E, Albrecht C, Barker JJ, Barker O, Beaumont E, Bromidge S, et al. Binding mode and structure-activity relationships around direct inhibitors of the Nrf2-Keap1 complex. ChemMedChem. 2014;9(4):699-705.

Karsten H, Pratap Singh P, Singh A, Chauhan S, Murat Singh Chauhan S. Activity-guided isolation of antioxidant xanthones from Swertia chirayita (Roxb.) H. Karsten (Gentianaceae). Nat. Prod. Res. [Internet]. 2012;26(18):1682-6. 
Kim S, Chen J, Cheng T, Gindulyte A, He J, He S, et al. PubChem 2019 update: Improved access to chemical data. Nucleic Acids Res. Oxford University Press; 2019 Jan 8;47(D1):D11029.

Koopmans T. Über die Zuordnung von Wellenfunktionen und Eigenwerten zu den Einzelnen Elektronen Eines Atoms. Physica. North-Holland; 1934 Jan 1;1(1-6):104-13.

Laskowski RA, Rullmann JAC, MacArthur MW, Kaptein R, Thornton JM. AQUA and PROCHECK-NMR: Programs for checking the quality of protein structures solved by NMR. J. Biomol. NMR [Internet]. Springer Netherlands; 1996;8(4):477-86.

Laskowski RA, Swindells MB. LigPlot+: Multiple ligand-protein interaction diagrams for drug discovery. J. Chem. Inf. Model. [Internet]. J Chem Inf Model; 2011 Oct 24;51(10):2778-86.

Lauritzen L, Brambilla P, Mazzocchi A, Harsløf LBS, Ciappolino V, Agostoni C. DHA effects in brain development and function [Internet]. Nutrients. MDPI AG; 2016.

Lee C, Yang W, Parr RG. Development of the Colle-Salvetti correlation-energy formula into a functional of the electron density. Phys. Rev. B [Internet]. American Physical Society; 1988 Jan 15;37(2):785-9.

Li M, Huang W, Jie F, Wang M, Zhong Y, Chen Q, et al. Discovery of Keap1-Nrf2 small-molecule inhibitors from phytochemicals based on molecular docking. Food Chem. Toxicol. [Internet]. Elsevier Ltd; 2019 Nov 1;133:110758.

Lipinski CA. Drug-like properties and the causes of poor solubility and poor permeability. J. Pharmacol. Toxicol. Methods [Internet]. 2000 Jul;44(1):235-49.

Liu H, Wang L, Lv M, Pei R, Li P, Pei Z, et al. AlzPlatform: An Alzheimer's disease domain-specific chemogenomics knowledgebase for polypharmacology and target identification research. J. Chem. Inf. Model. [Internet]. American Chemical Society; 2014;54(4):1050-60.

Meanwell NA. Synopsis of some recent tactical application of bioisosteres in drug design [Internet]. J. Med. Chem. J Med Chem; 2011. p. 2529-91.

Müller K, Faeh C, Diederich F. Fluorine in pharmaceuticals: Looking beyond intuition [Internet]. Science (80-. ). Science; 2007. p. 1881-6.

Murugavel S, Ravikumar C, Jaabil G, Alagusundaram P. Synthesis, crystal structure analysis, spectral investigations (NMR, FT-IR, UV), DFT calculations, ADMET studies, molecular docking and anticancer activity of 2-(1-benzyl-5-methyl-1H-1,2,3-triazol-4-yl)-4-(2chlorophenyl)-6-methoxypyridine - A novel poten. J. Mol. Struct. Elsevier B.V.; 2019;1176:72942 .

Napolitano A, Manini P, D'Ischia M. Oxidation Chemistry of Catecholamines and Neuronal Degeneration: An Update. Curr. Med. Chem. 2011;18(12):1832-45.

Napolitano A, Pezzella A, Prota G. 6,7-Dihydroxy-1,2,3,4-tetrahydroisoquinoline formation by iron mediated dopamine oxidation: A novel route to endogenous neurotoxins under oxidative stress conditions. Tetrahedron Lett. 1999a;40(14):2833-6.

Napolitano A, Pezzella A, Prota G. New reaction pathways of dopamine under oxidative stress conditions: Nonenzymatic iron-assisted conversion to norepinephrine and the neurotoxins 6-hydroxydopamine and 6,7-dihydroxytetrahydroisoquinoline. Chem. Res. Toxicol.1999b;12(11):1090-7.

Nelson SK, Bose SK, Grunwald GK, Myhill P, McCord JM. The induction of human superoxide dismutase and catalase in vivo: A fundamentally new approach to antioxidant therapy. 
Free Radic. Biol. Med. 2006;40(2):341-7.

Parr RG, Pearson RG. Absolute Hardness: Companion Parameter to Absolute Electronegativity. J. Am. Chem. Soc. [Internet]. American Chemical Society; 1983;105(26):7512- 6.

Parr RG, Yang W. Density-Functional Theory of the Electronic Structure of Molecules. Annu. Rev. Phys. Chem. [Internet]. Annual Reviews; 1995 Oct;46(1):701-28.

Pettersen EF, Goddard TD, Huang CC, Couch GS, Greenblatt DM, Meng EC F TE. UCSF Chimera--a visualization system for exploratory research and analysis. J Comput Chem. 2004.

Purser S, Moore PR, Swallow S, Gouverneur V. Fluorine in medicinal chemistry. Chem. Soc. Rev. Chem Soc Rev; 2008 Jan 7;37(2):320-30.

Ross BM, McKenzie I, Glen I, Bennett CPW. Increased levels of ethane, a non-invasive marker of n-3 fatty acid oxidation, in breath of children with attention deficit hyperactivity disorder. Nutr. Neurosci. 2003;6(5):277-81.

Saito R, Suzuki T, Hiramoto K, Asami S, Naganuma E, Suda H, et al. Characterizations of Three Major Cysteine Sensors of Keap1 in Stress Response. 2016;

Shaw C-Y, Chen C-H, Hsu C-C, Chen C-C, Tsai Y-C. Antioxidant properties of scopoletin isolated fromSinomonium acutum. Phyther. Res. [Internet]. John Wiley \& Sons, Ltd; 2003 Aug $1 ; 17(7): 823-5$.

Shukla S, Sumaria CS, Pradeepkumar PI. Exploring chemical modifications for siRNA therapeutics: A structural and functional outlook. ChemMedChem. 2010;5(3):328-49.

Sonarthi H, Sivakumar S, Chawla R, Veena N, Mishra SK, Singh L, et al. Quality Characteristics of Functional Yoghurt with Fortification of Encapsulated Resveratrol Powder. Int.J.Curr.Microbiol.App.Sci [Internet]. 2018;7(8):3102-9.

Trott O, Olson AJ. AutoDock Vina: Improving the speed and accuracy of docking with a new scoring function, efficient optimization, and multithreading. J. Comput. Chem. [Internet]. Wiley; 2009 Jan 30;31(2):NA-NA.

Uzzaman M, Mahmud T. Structural modification of aspirin to design a new potential cyclooxygenase (COX-2) inhibitors. Silico Pharmacol. [Internet]. Springer Berlin Heidelberg; 2020;8(1).

Wilde MI, Plosker GL, Benfield P. Fluvoxamine: An Updated Review of its Pharmacology, and Therapeutic Use in Depressive Illness. Drugs [Internet]. Springer; 1993 Oct 22;46(5):895-924.

Yakubenko VP, Byzova T V. Biological and pathophysiological roles of end-products of DHA oxidation. Biochim. Biophys. Acta - Mol. Cell Biol. Lipids [Internet]. Elsevier B.V.; 2017;1862(4):407-15.

Yang X, Kang M-C, Lee K-W, Kang S-M, Lee W-W, Jeon Y-J. Antioxidant activity and cell protective effect of loliolide isolated from Sargassum ringgoldianum subsp. coreanum. Algae. 2011;26(2):201-8 


\section{Supplementary information}

Figure S1: The color-coded contour maps illustrating the Highest occupied molecular orbitals(HOMO) and the lowest unoccupied molecular orbital (LUMO) of the entire ligand library.

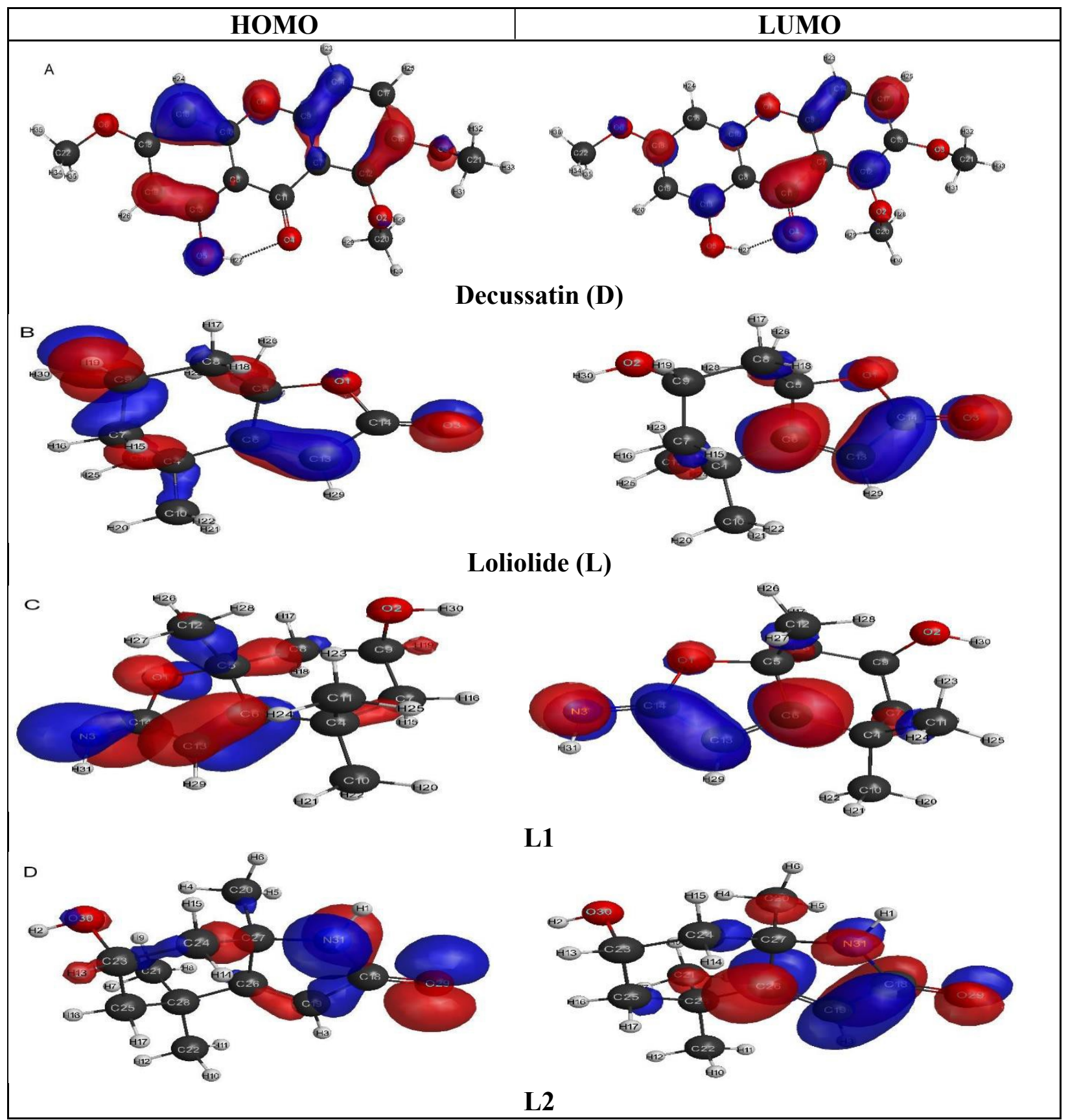




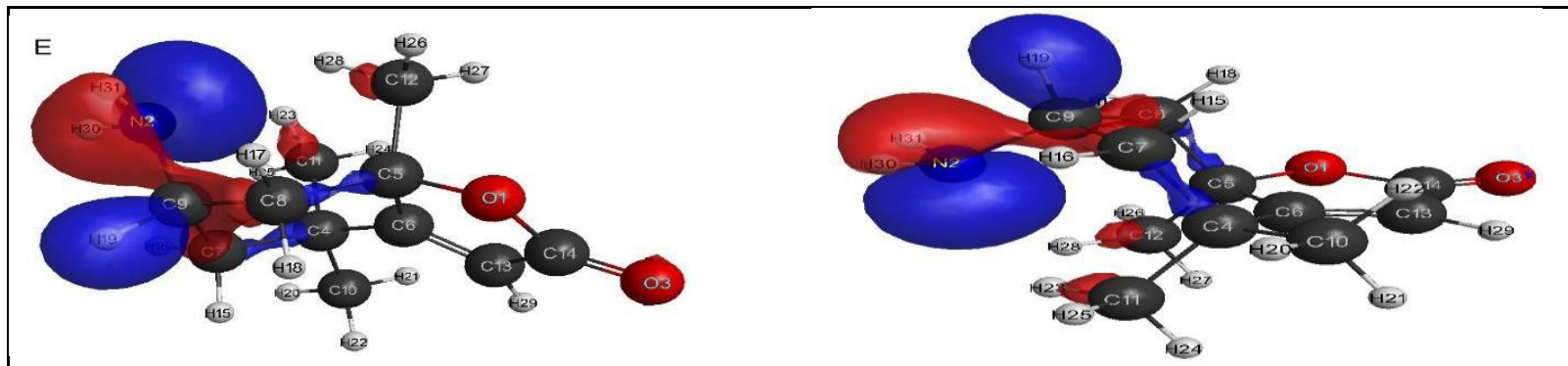

L3
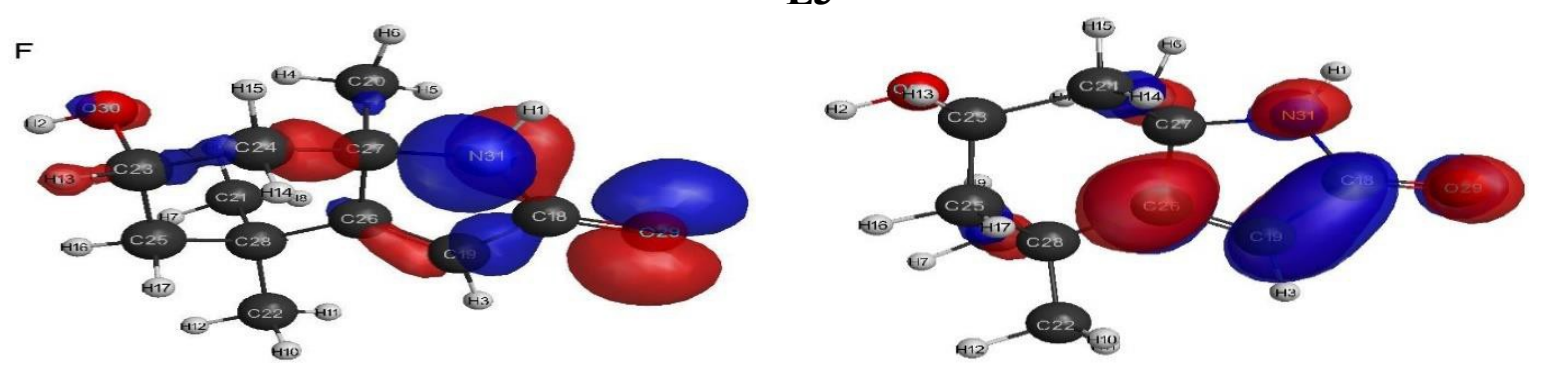

L4
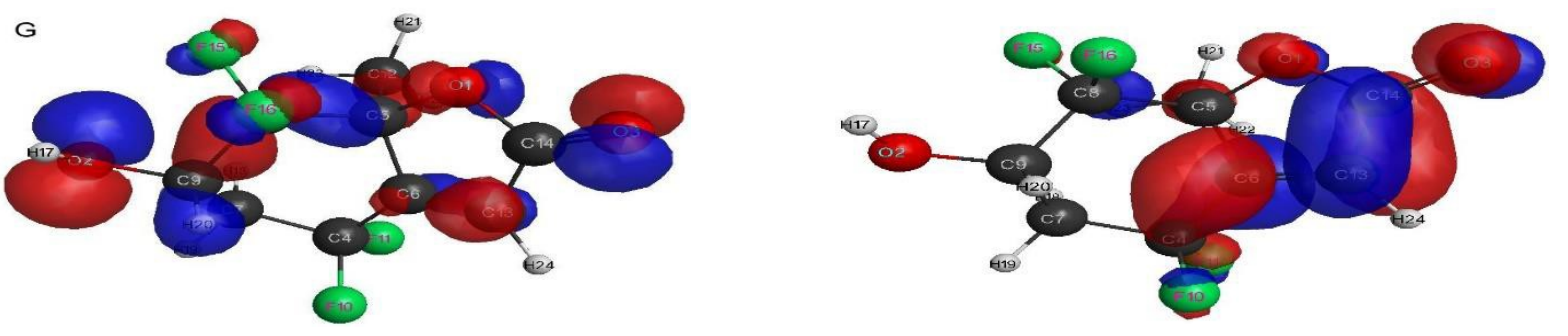

L5
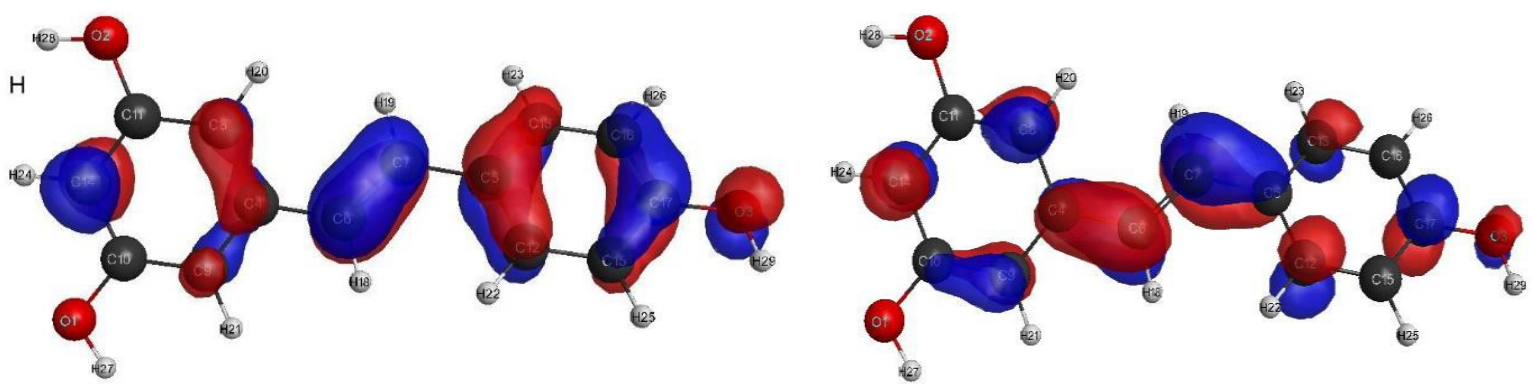

Resveratrol

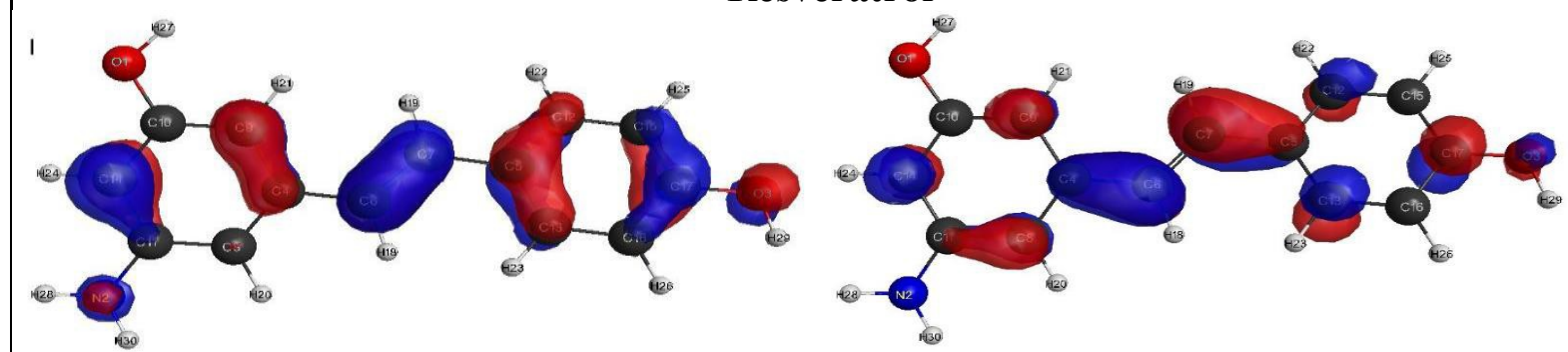

R1 


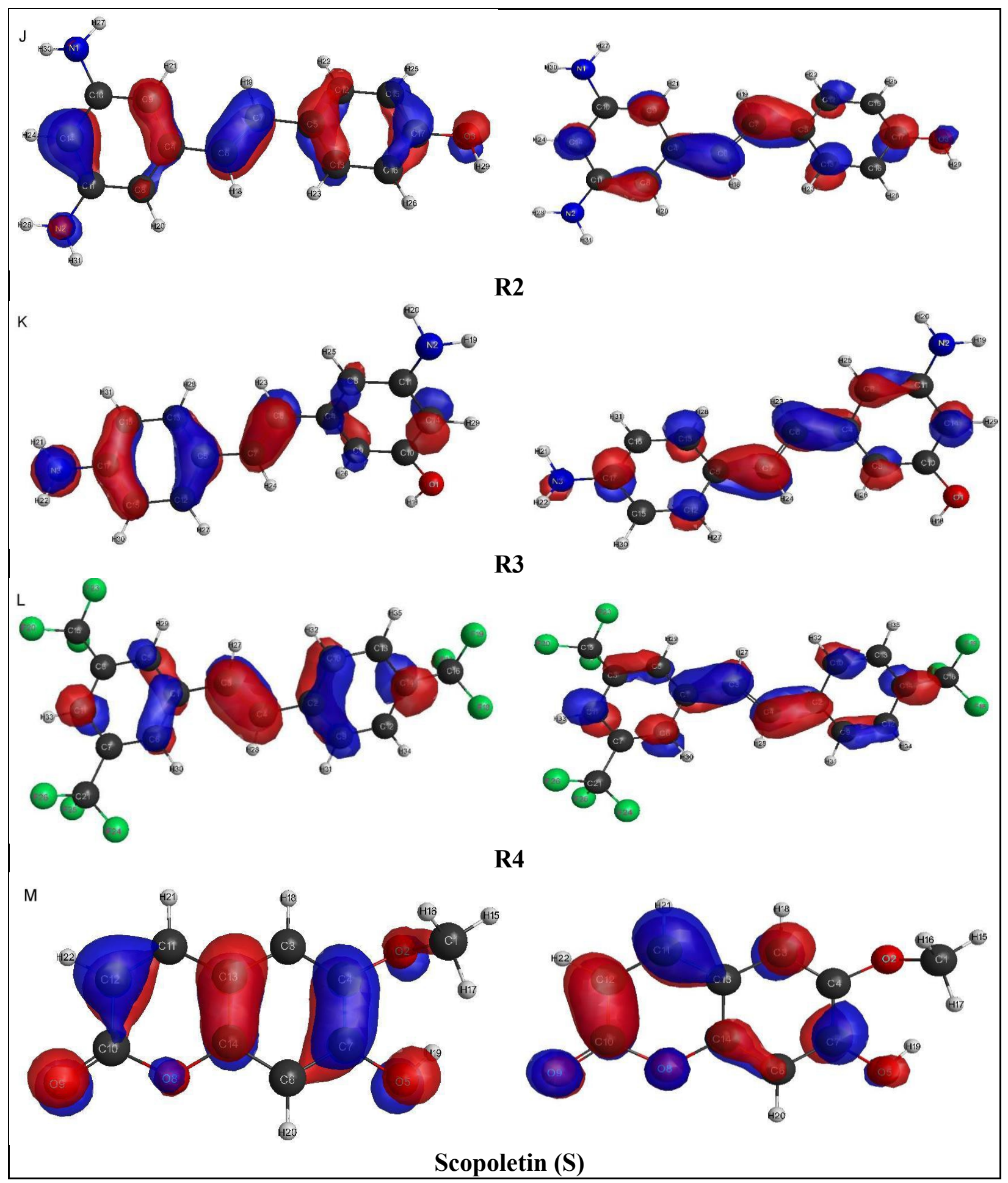




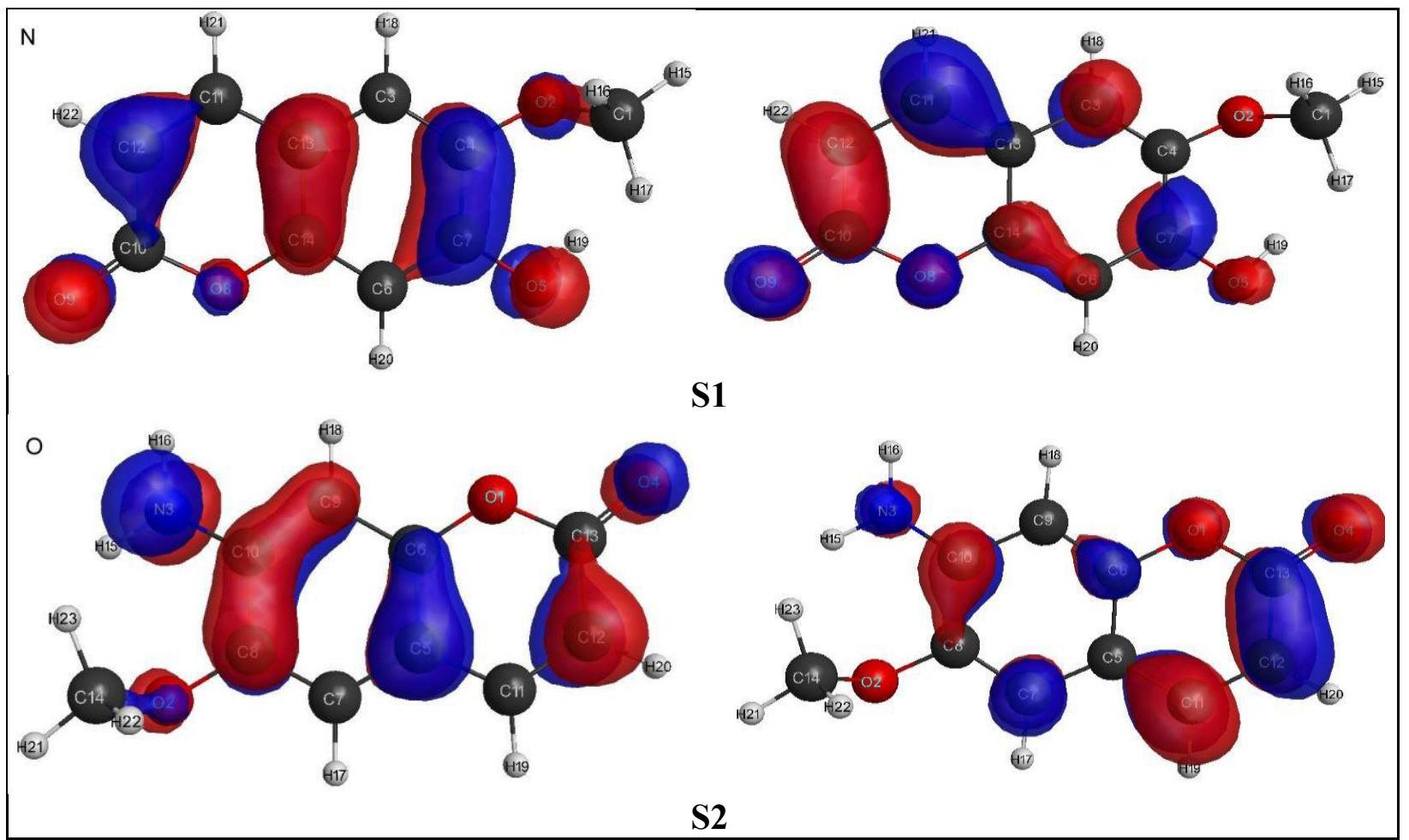

Figure S2: The color-coded contour maps illustrating the variation of molecular electrostatic potentials over the regions of the ligands in the constructed library.

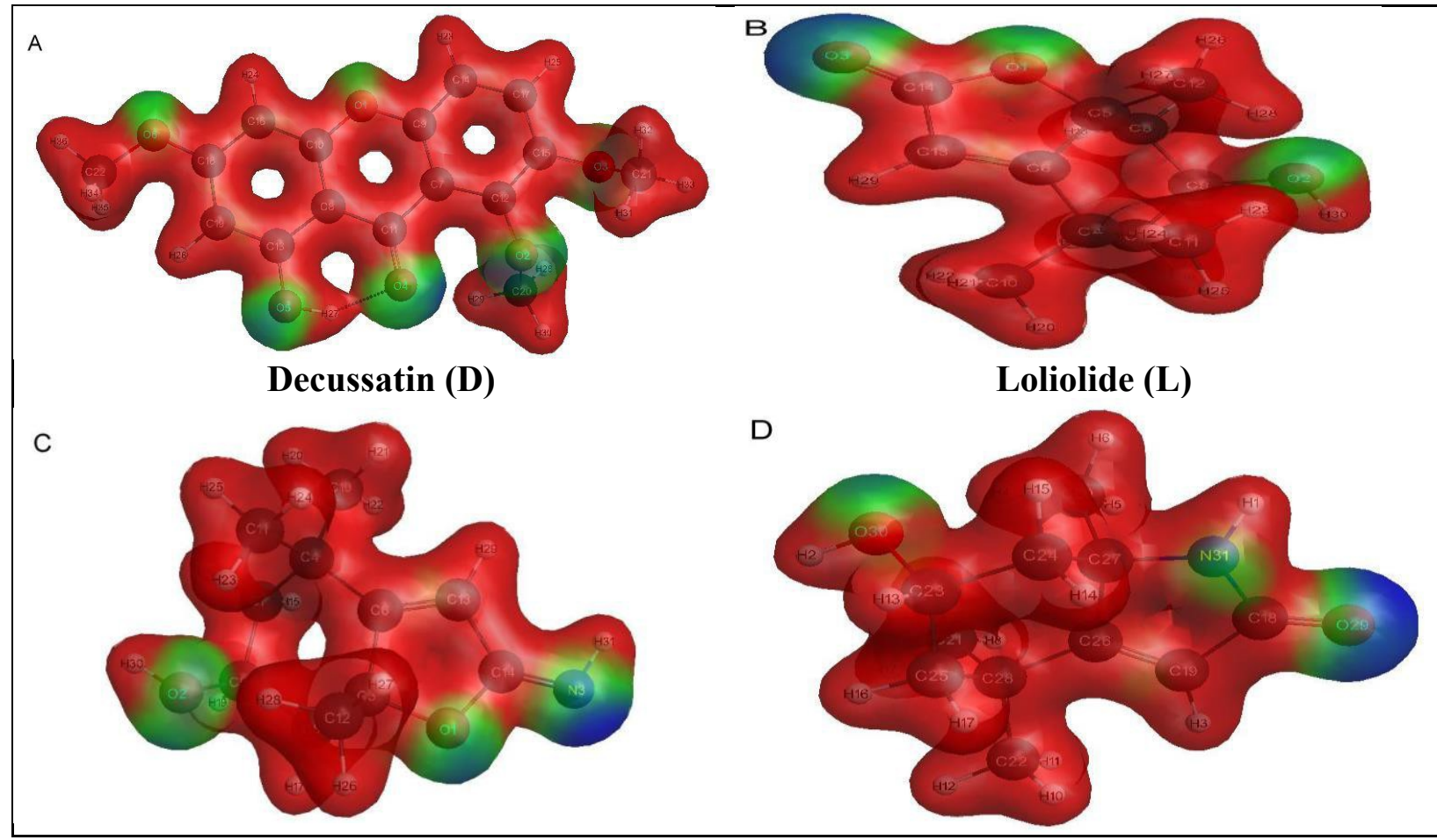




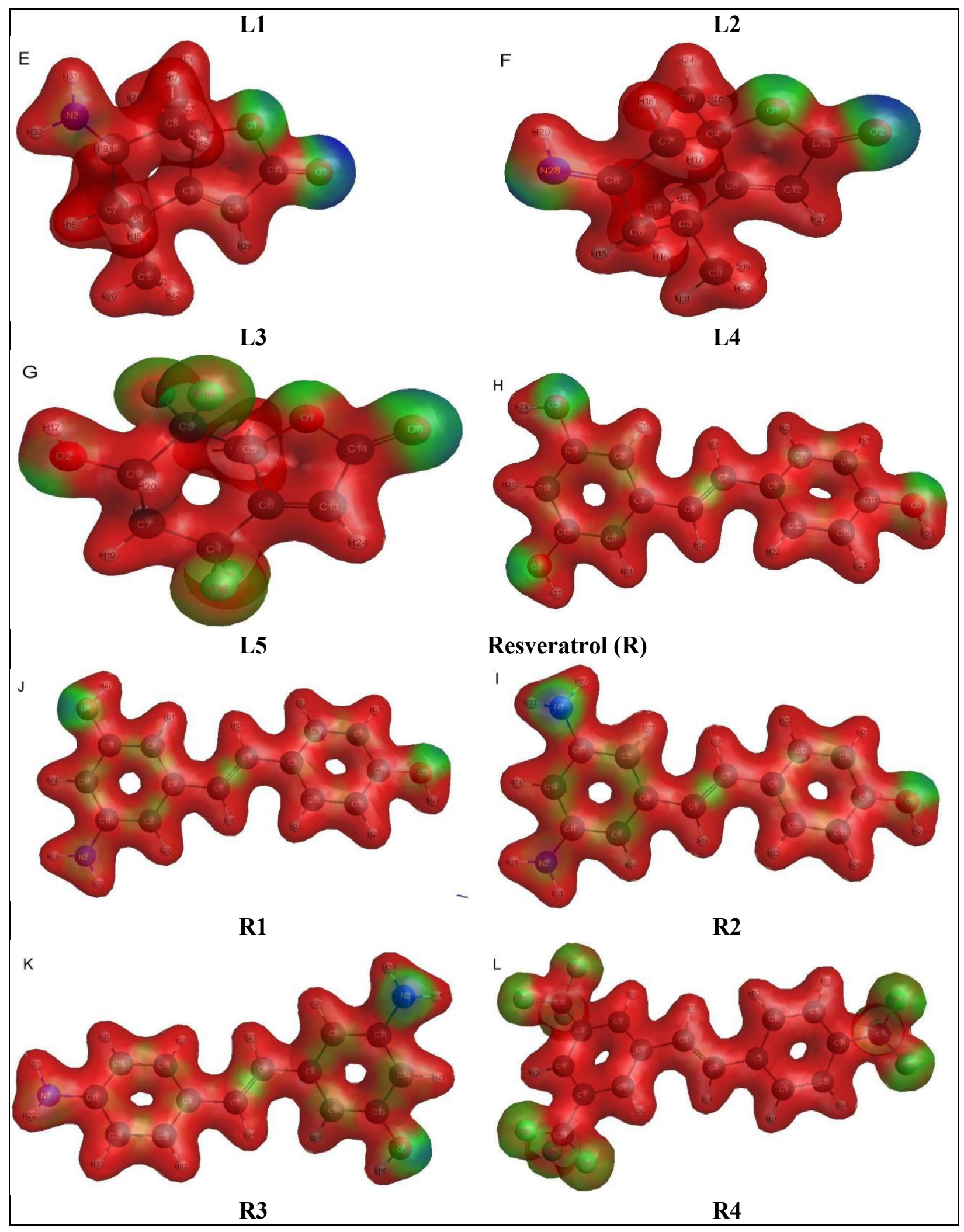




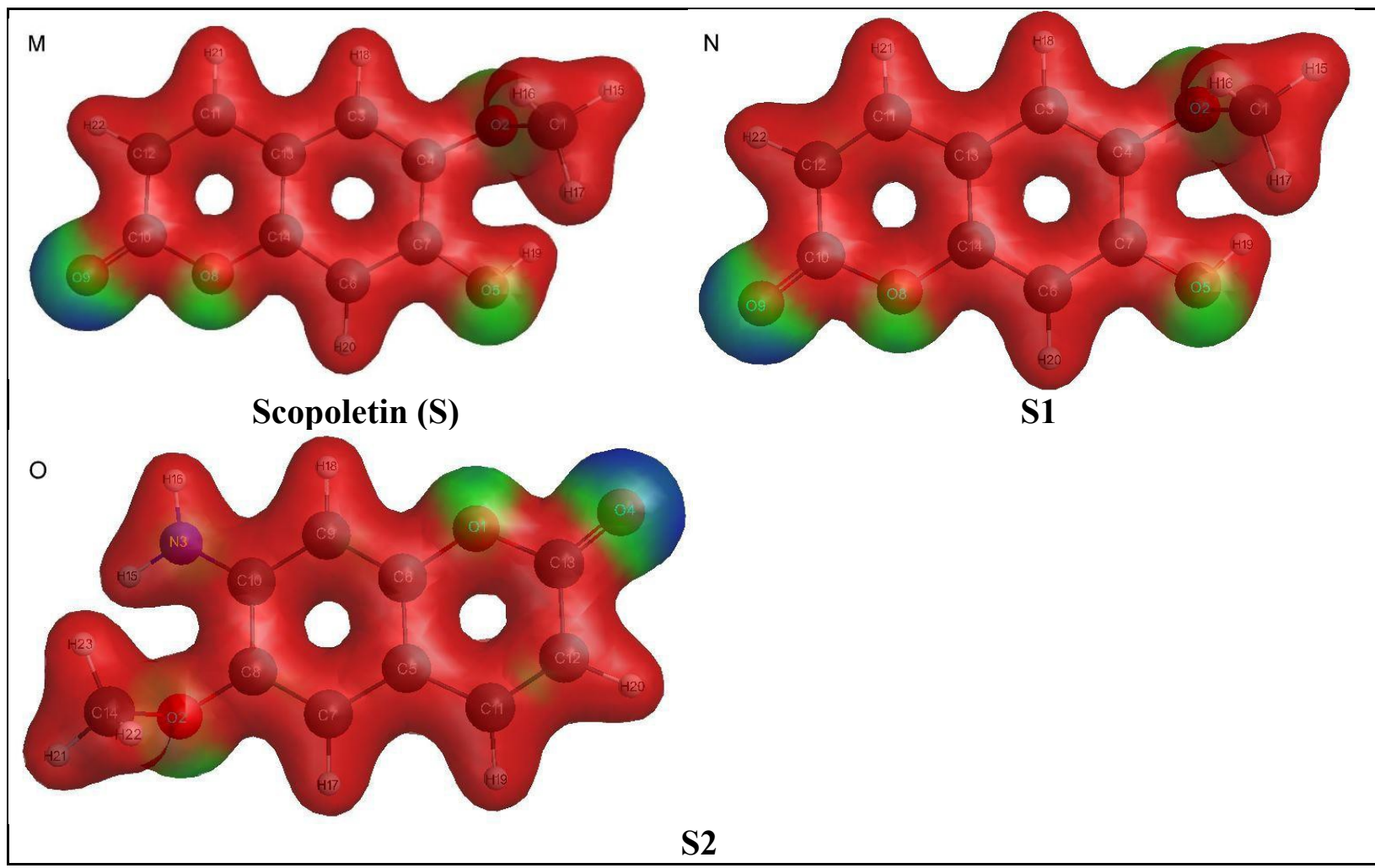

Figure S3: The two-dimensional illustrations of the interaction analysis of the individual ligands with the BTB domain of the Keap1 protein as obtained from LigPlot.

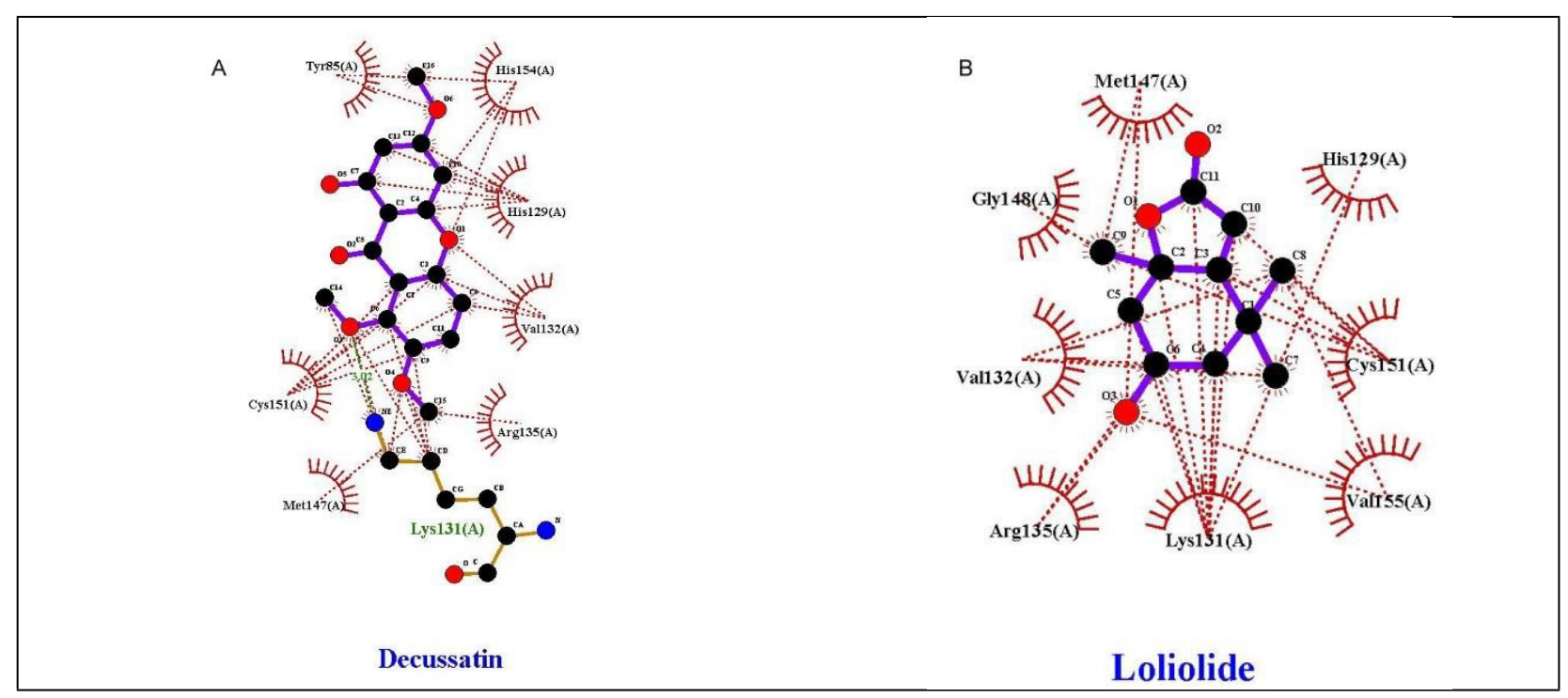




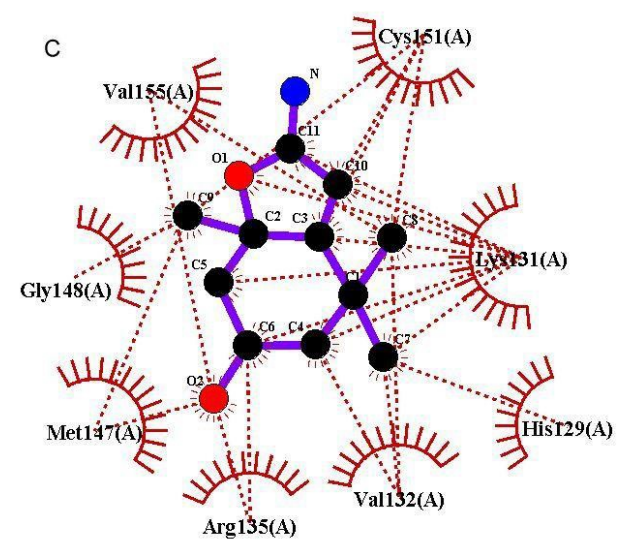

Loliolide (L1)

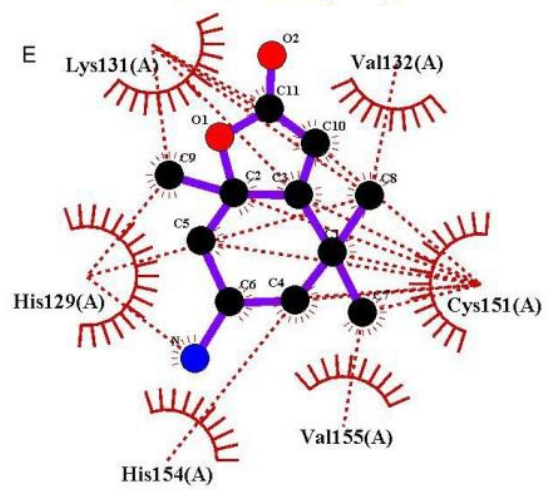

Loliolide (L3)

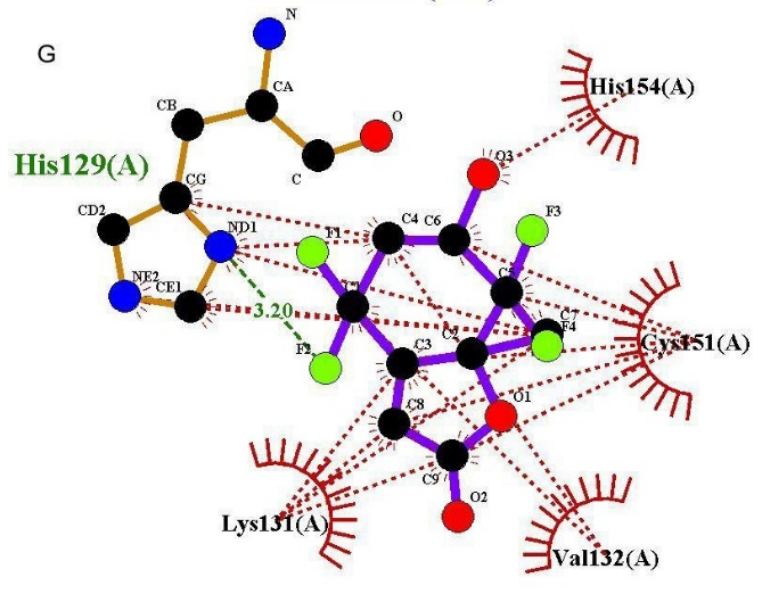

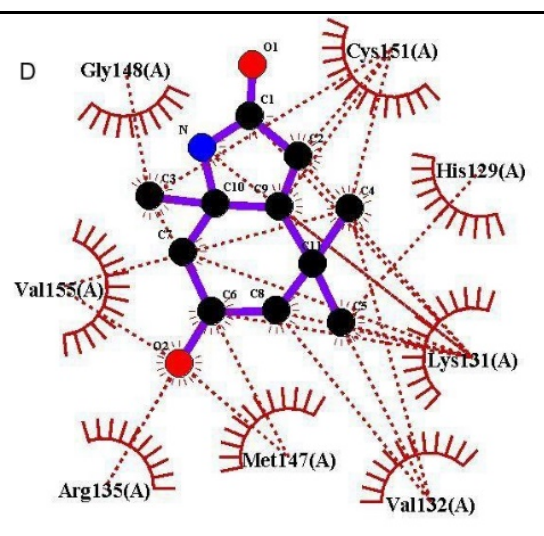

Loliolide (L2)

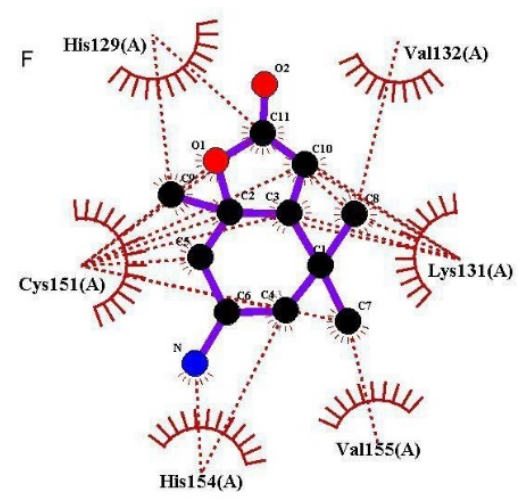

Loliolide (L4)

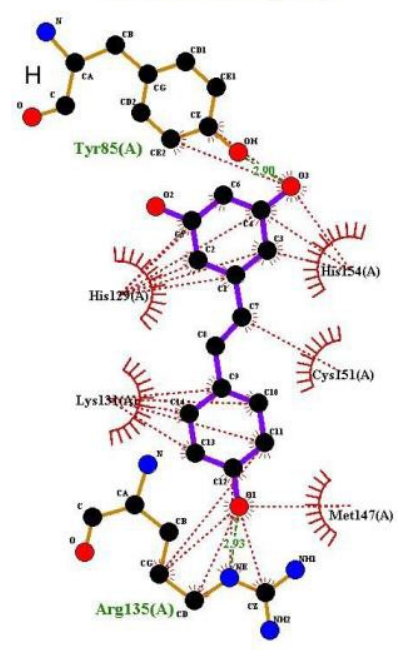



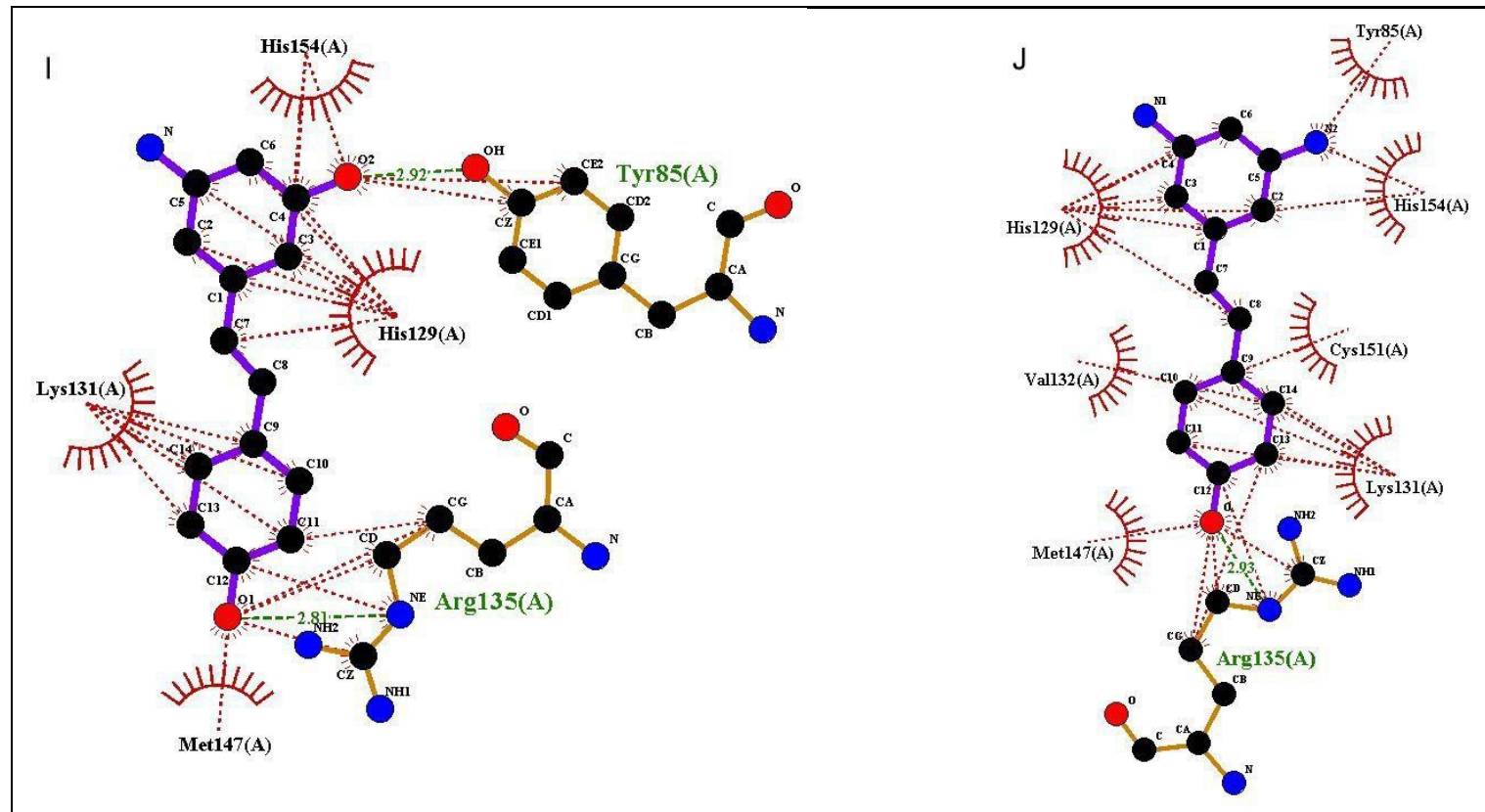

Resveratrol (R1)

Resveratrol (R2)
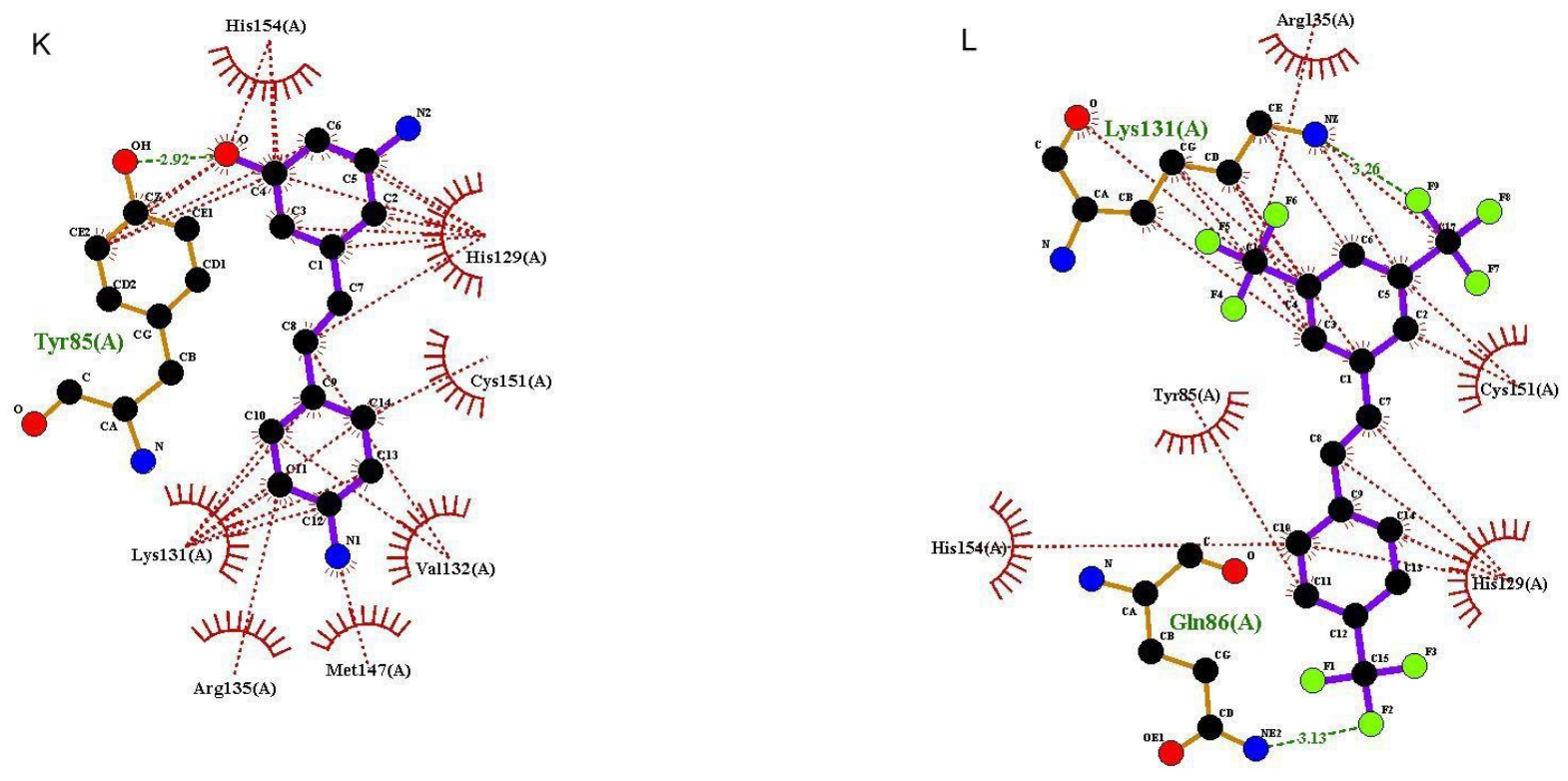

Resveratrol (R3) 

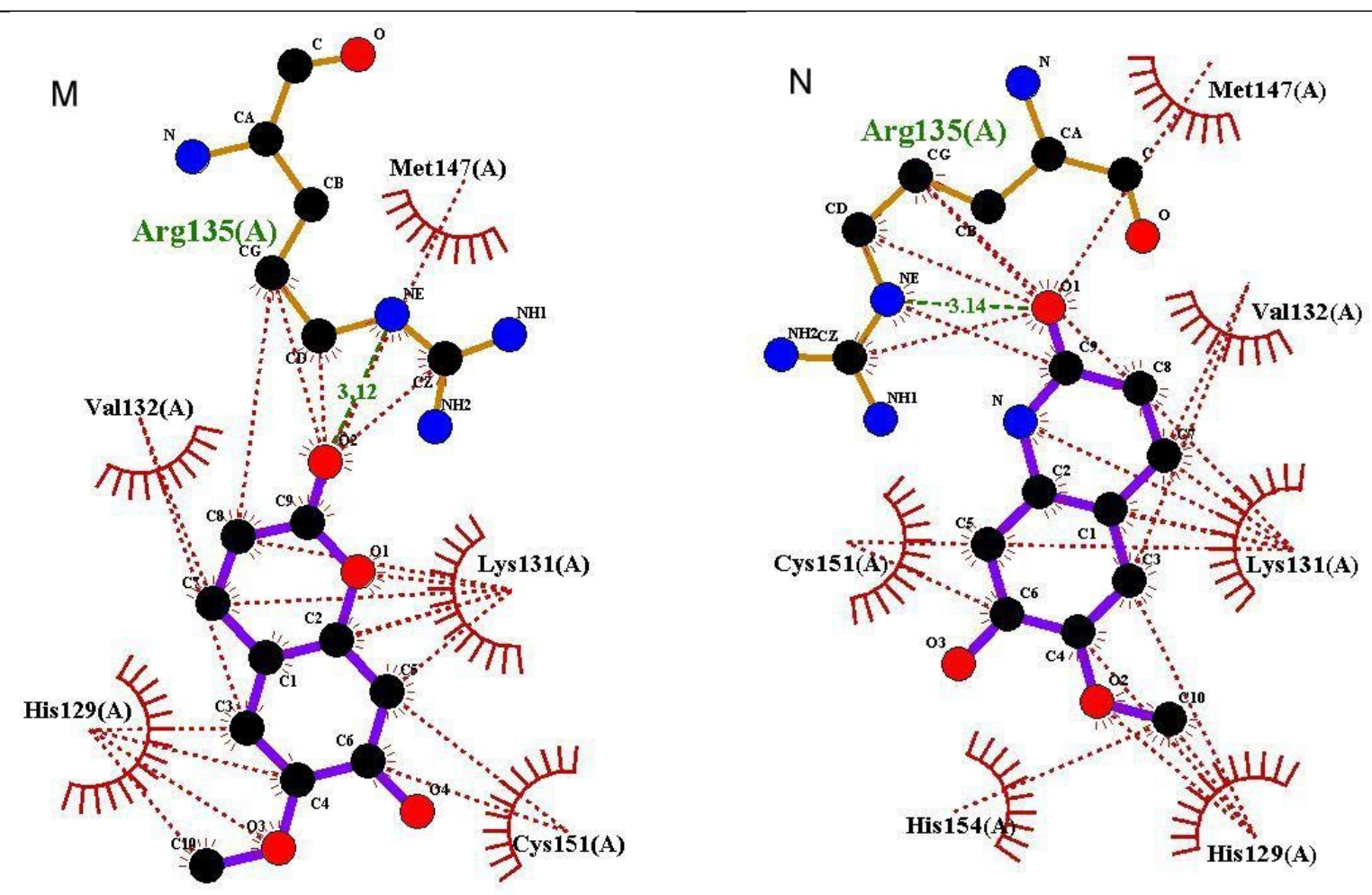

Scopoletin (S)

Scopoletin (S1)

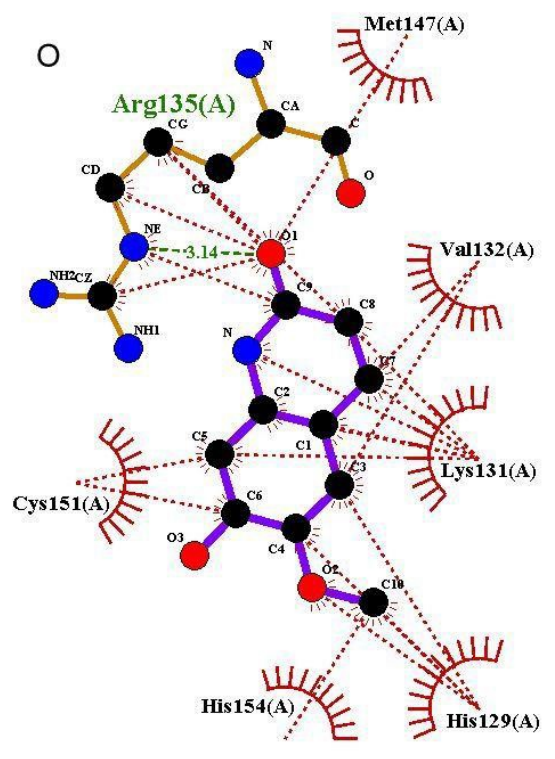

Scopoletin (S2) 
Figure S4: The two-dimensional illustrations of the interaction analysis of the individual ligands with the Kelch domain of the Keap1 protein as obtained from LigPlot.

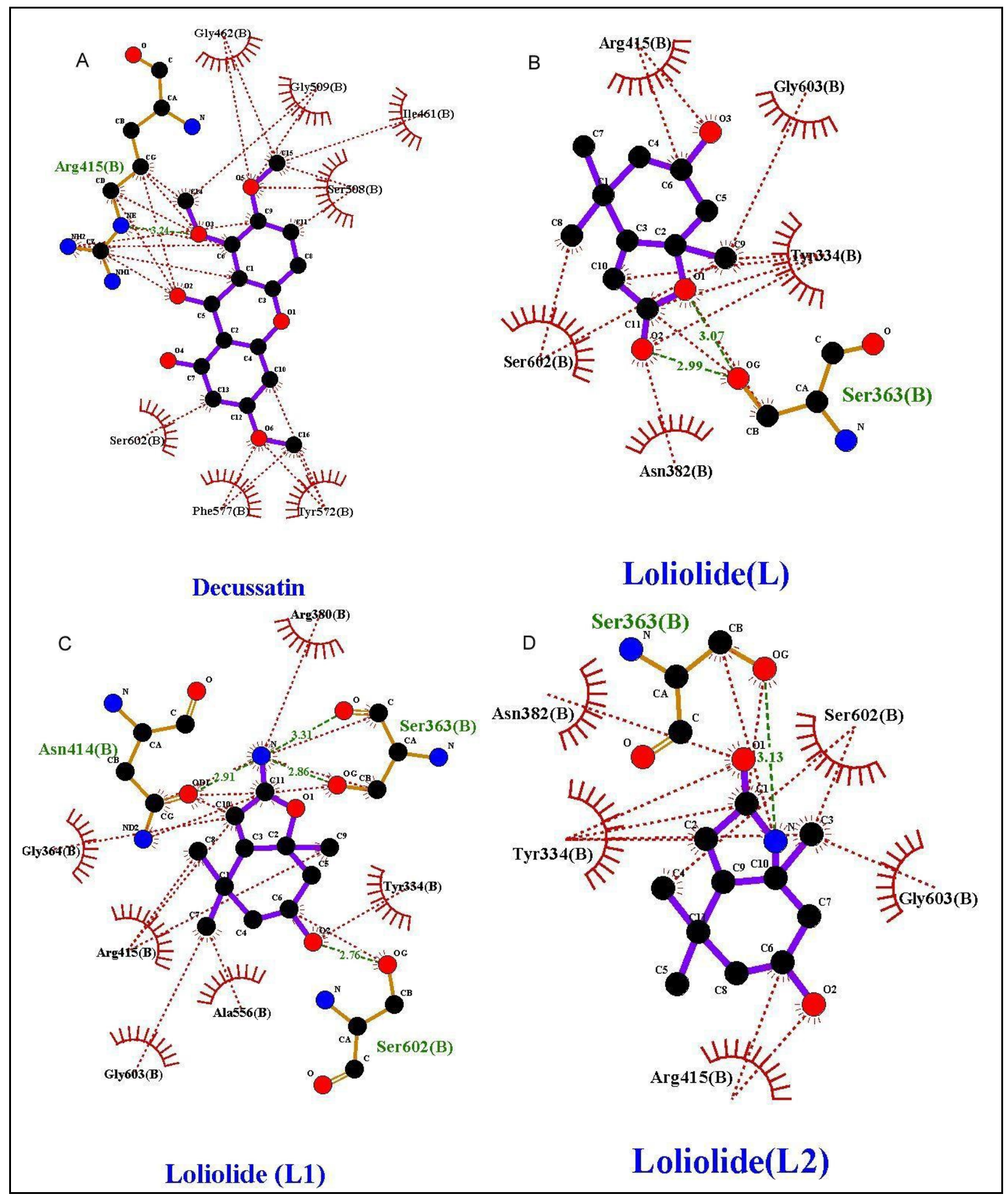




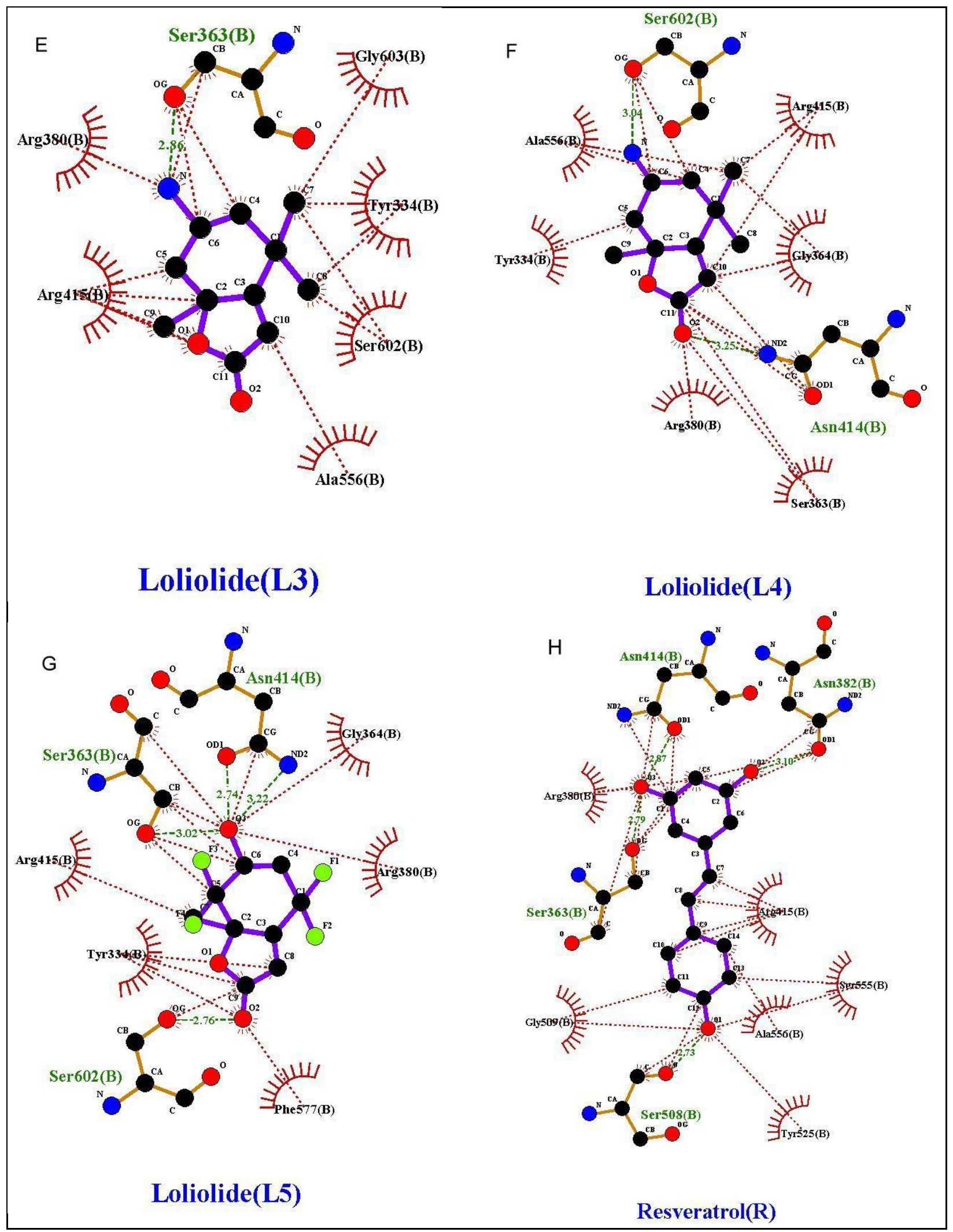



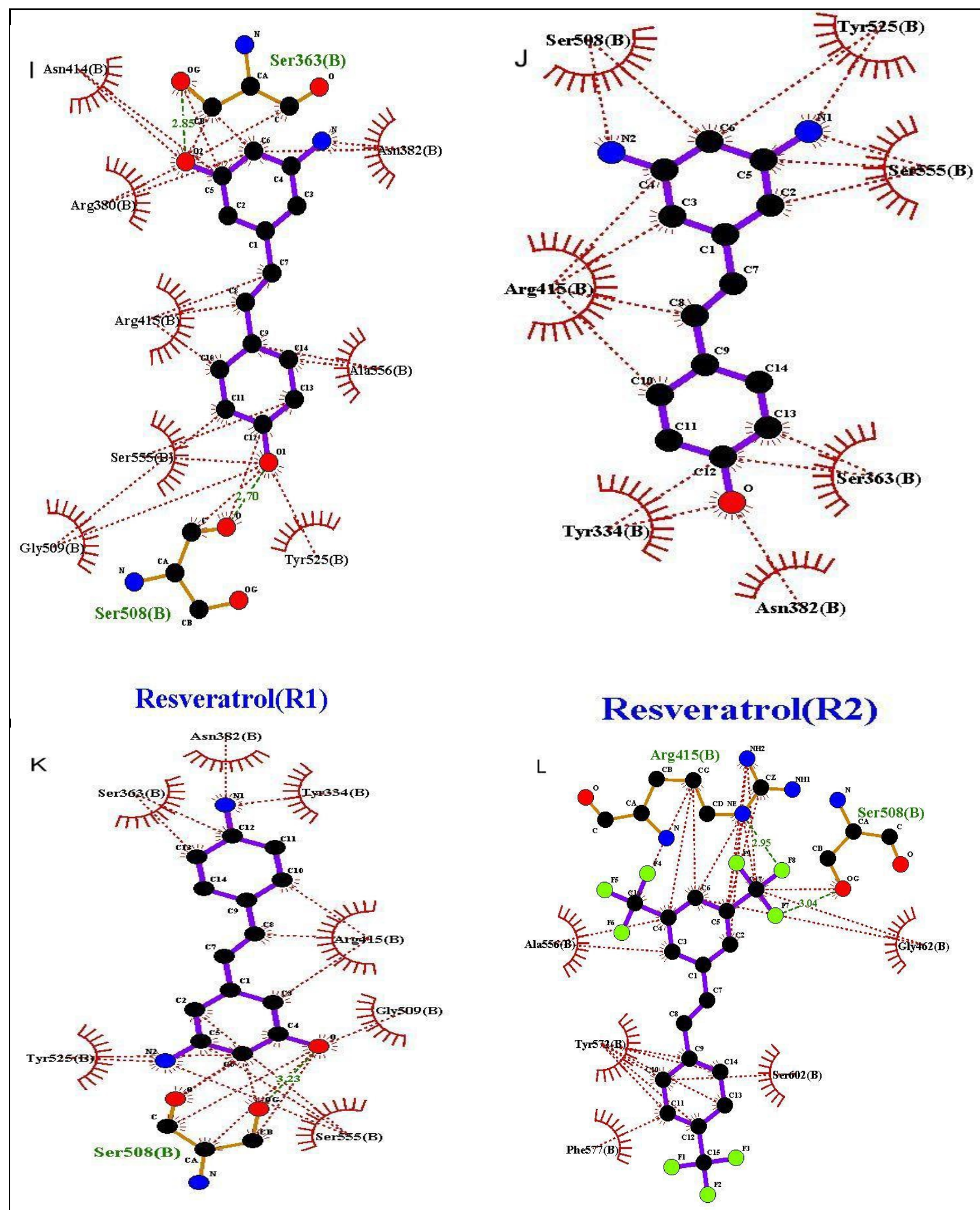

Resveratrol (R3) 


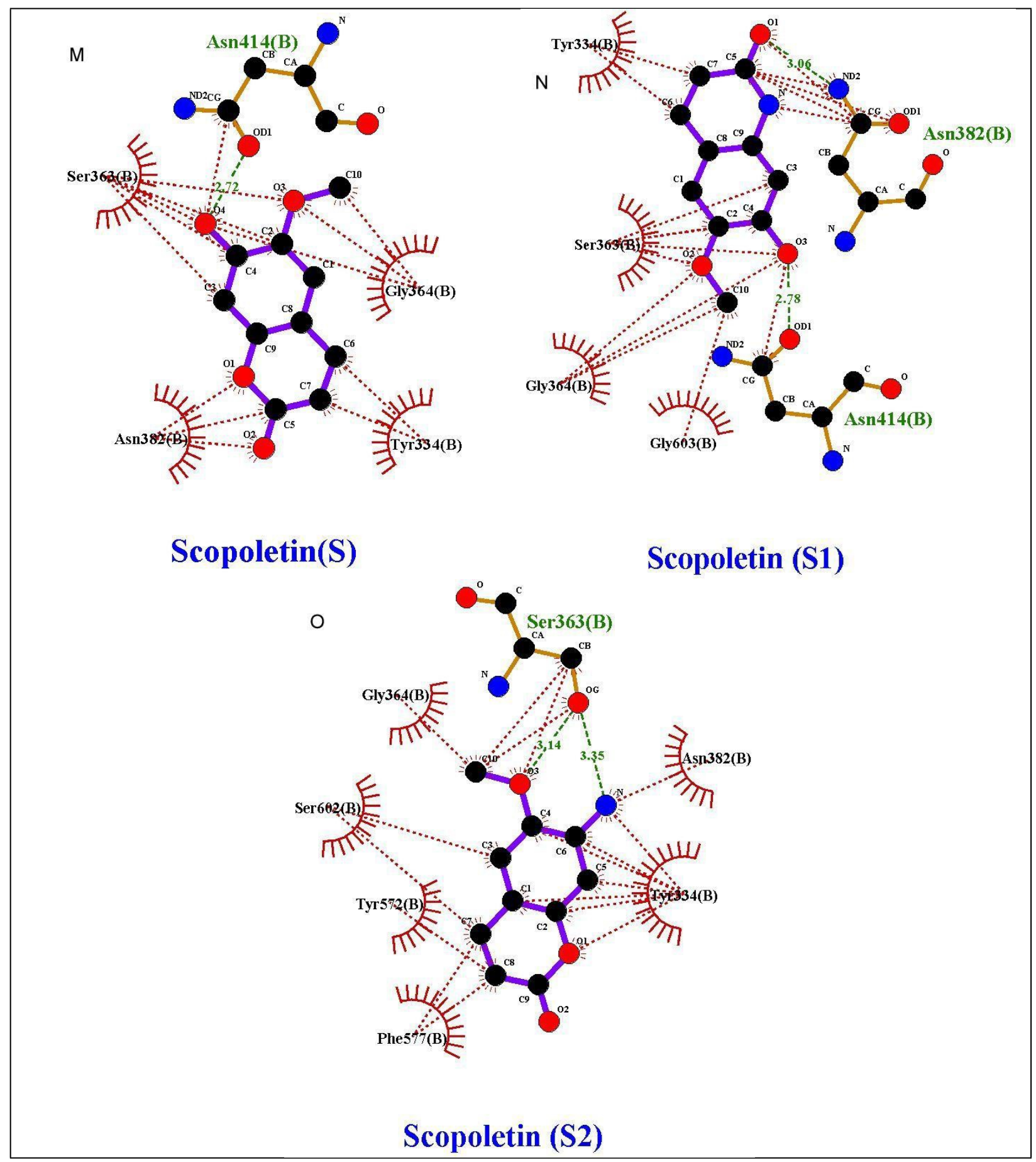


Figure S5: The three-dimensional illustrations of the interaction analysis of the individual ligands with the BTB domain of the Keap1 protein as obtained from the Discovery studio and the surface view of the docked ligand in the BTB active site as obtained from PyMol.

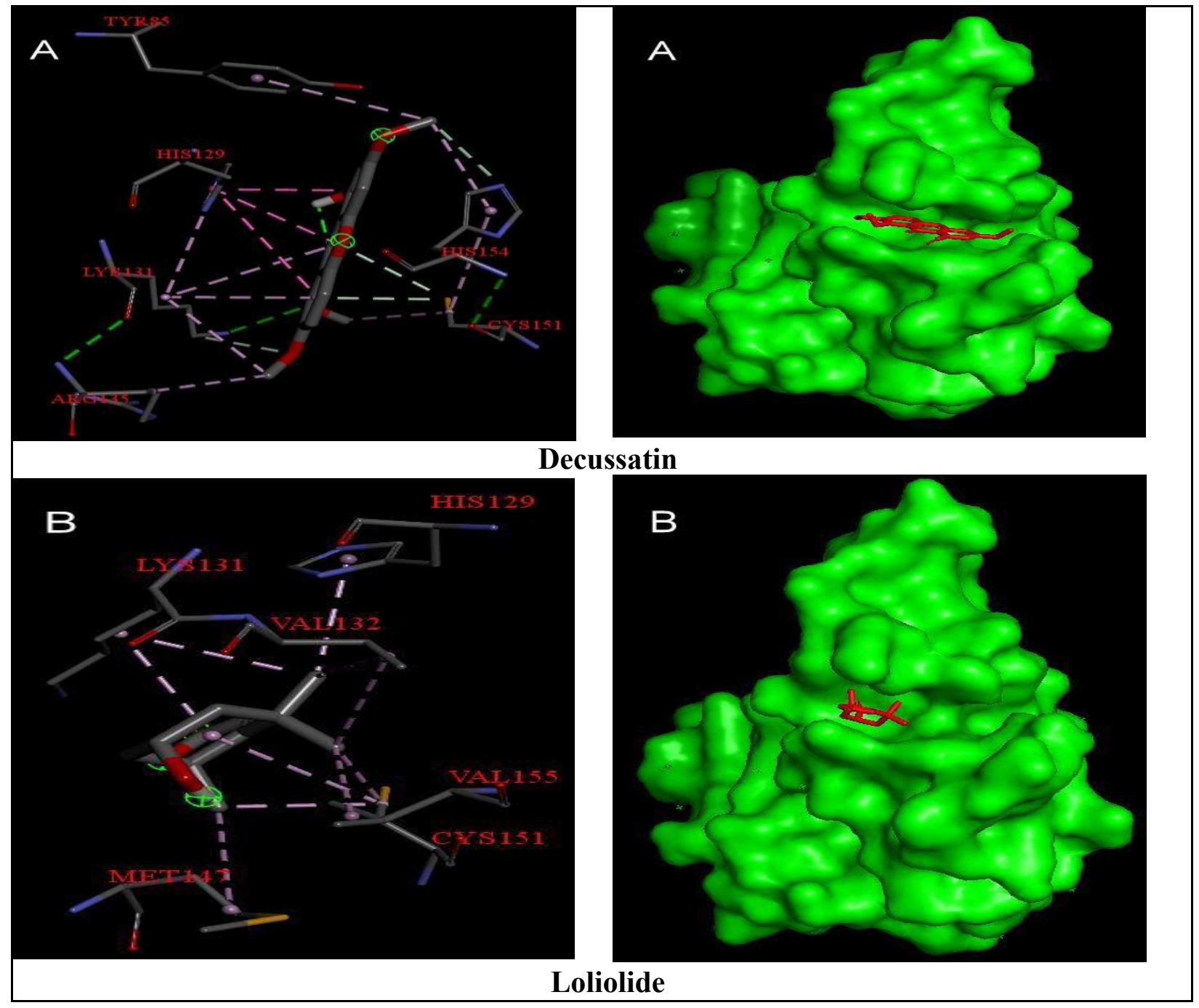




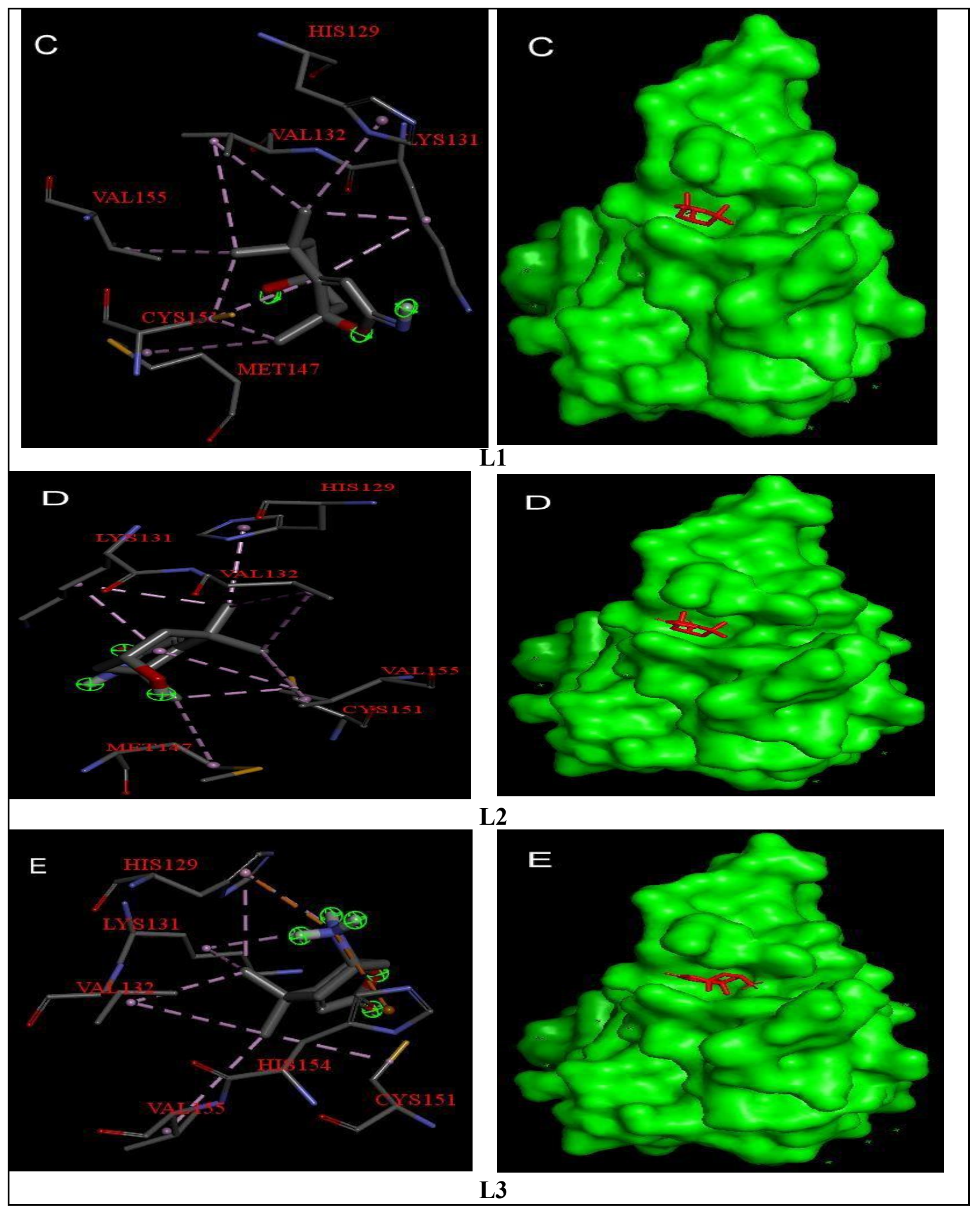




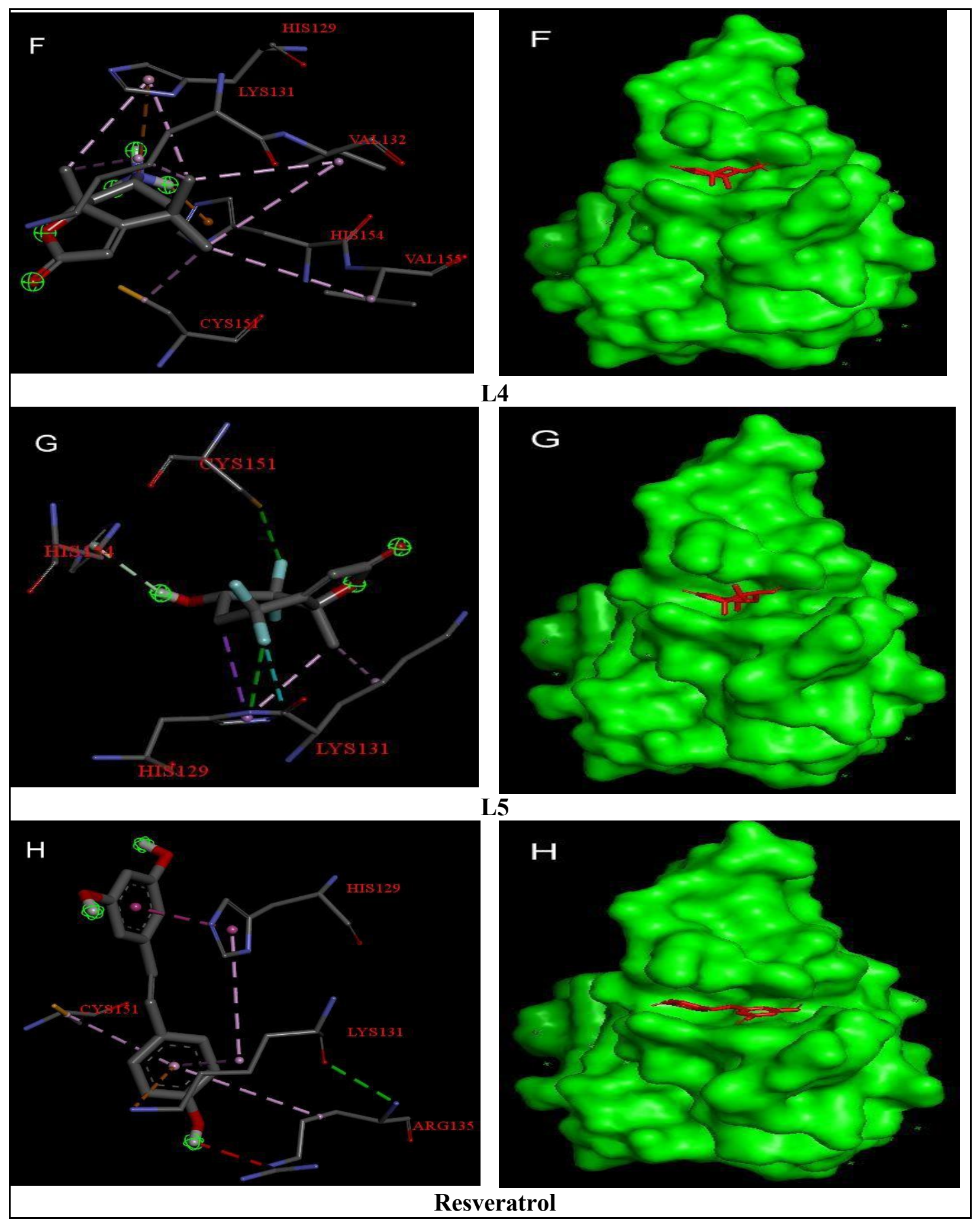




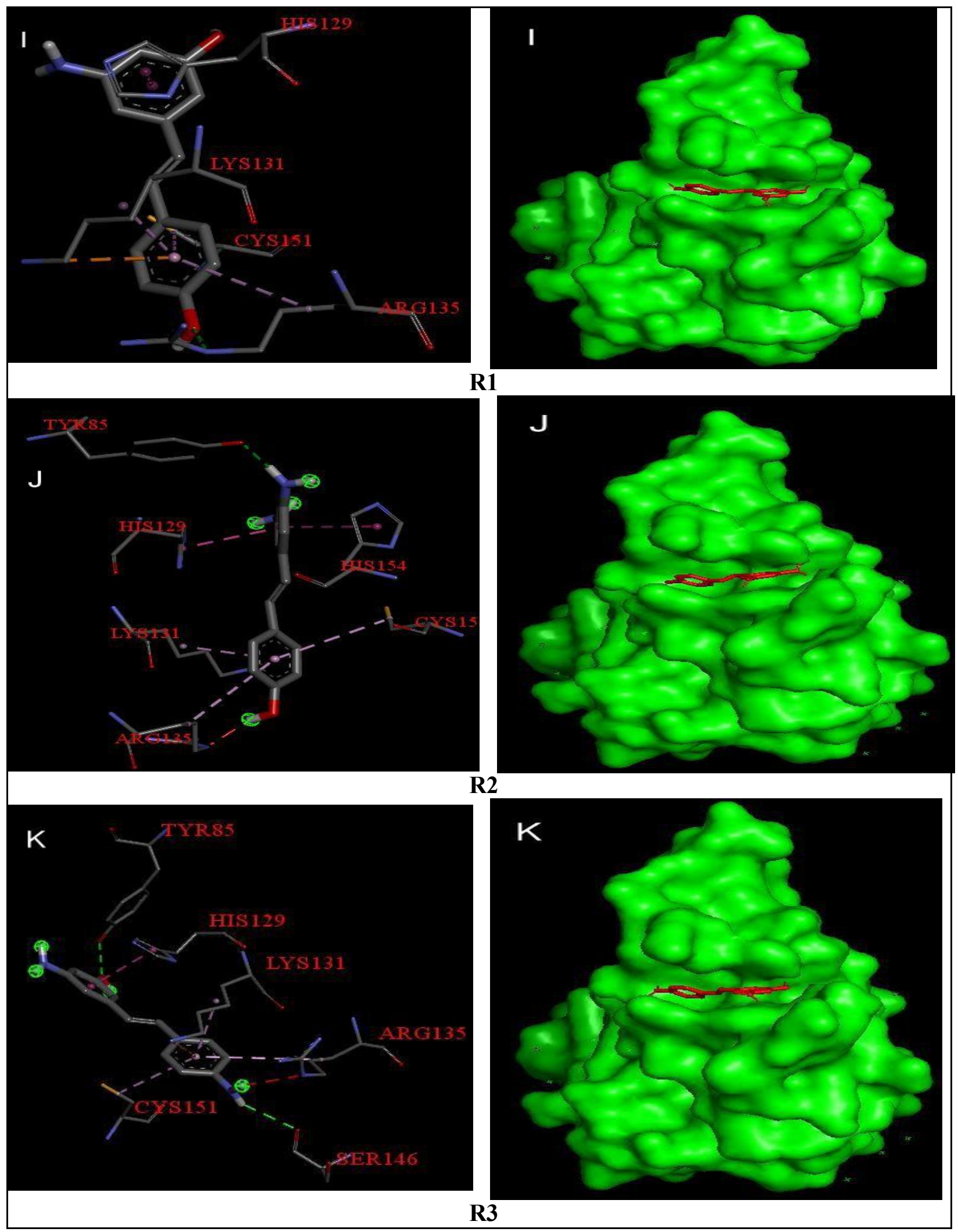




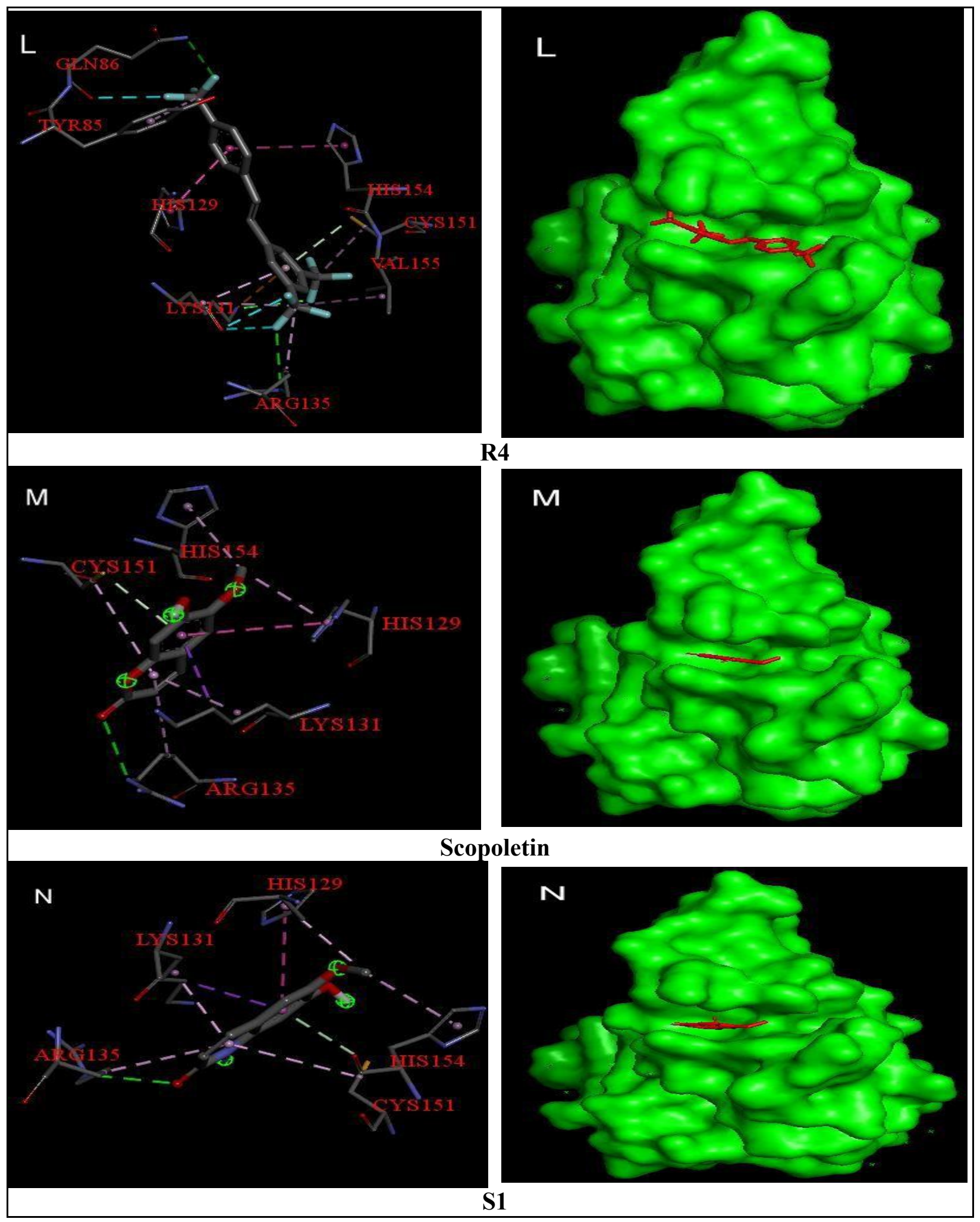




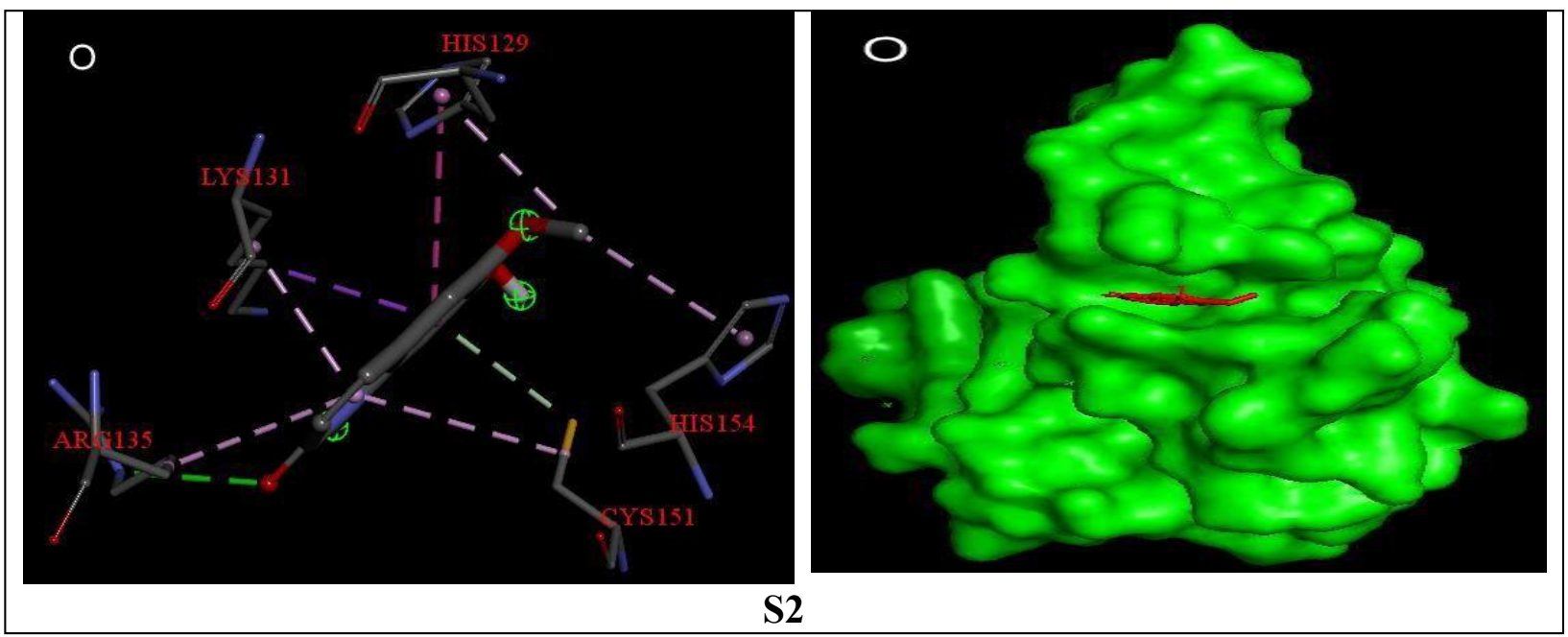

Figure S6: The three-dimensional illustrations of the interaction analysis of the individual ligands with the Kelch domain of the Keap1 protein as obtained from the Discovery studio and the surface view of the docked ligand in the BTB active site as obtained from PyMol.

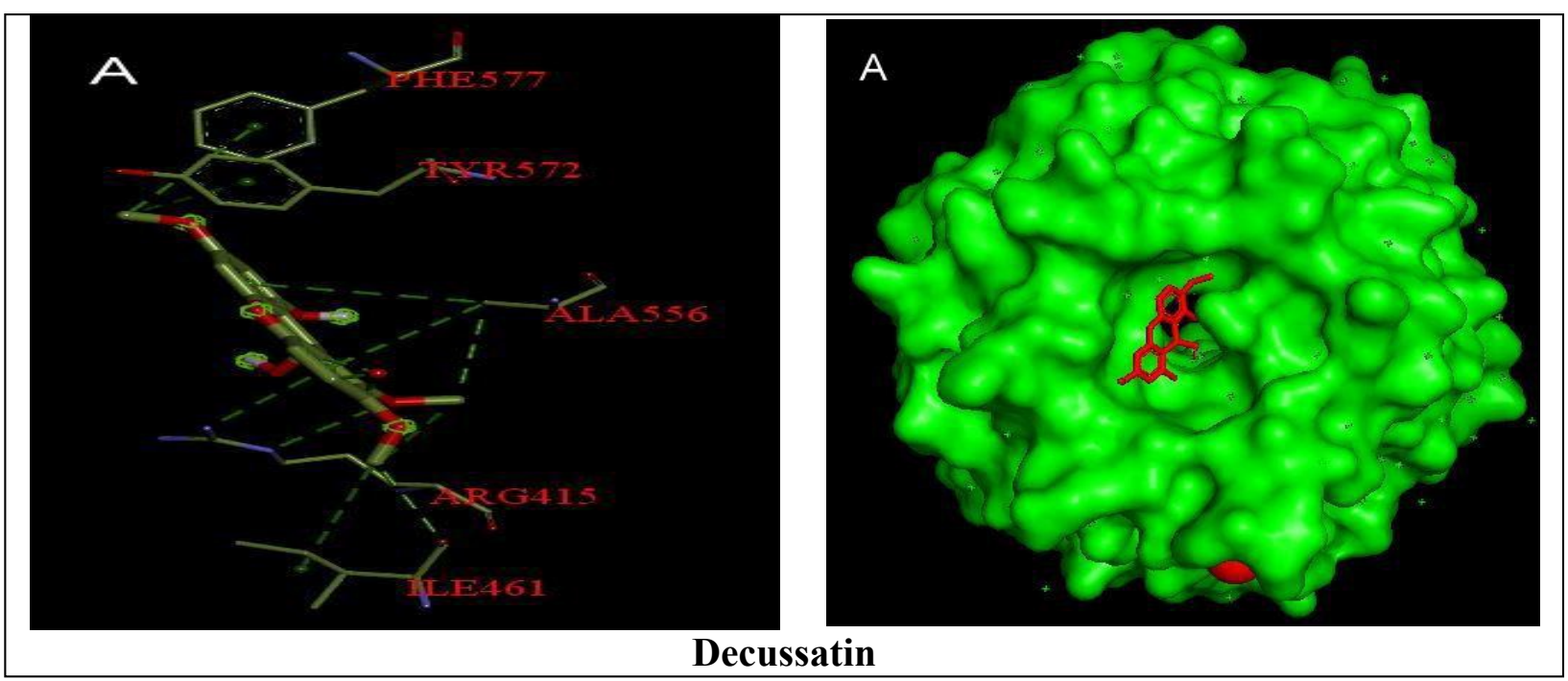




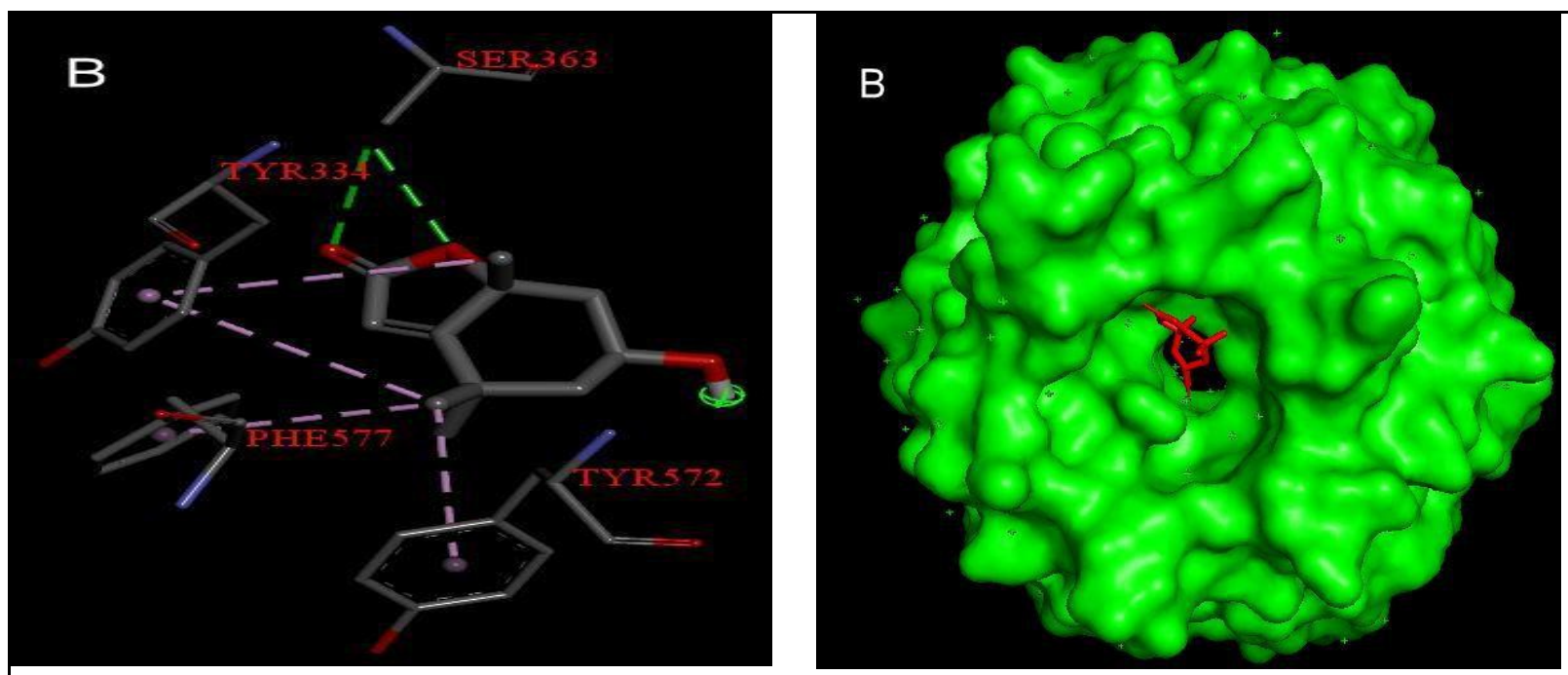

Loliolide
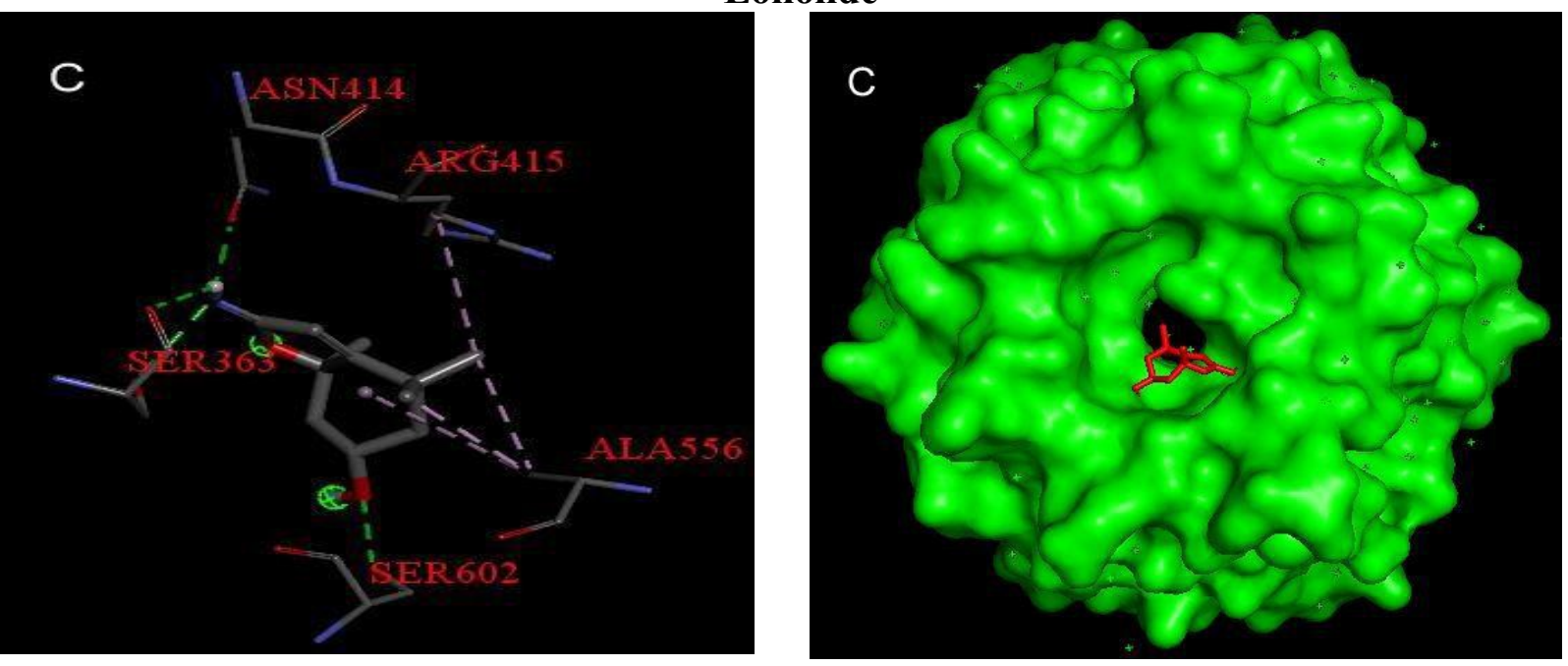

L1
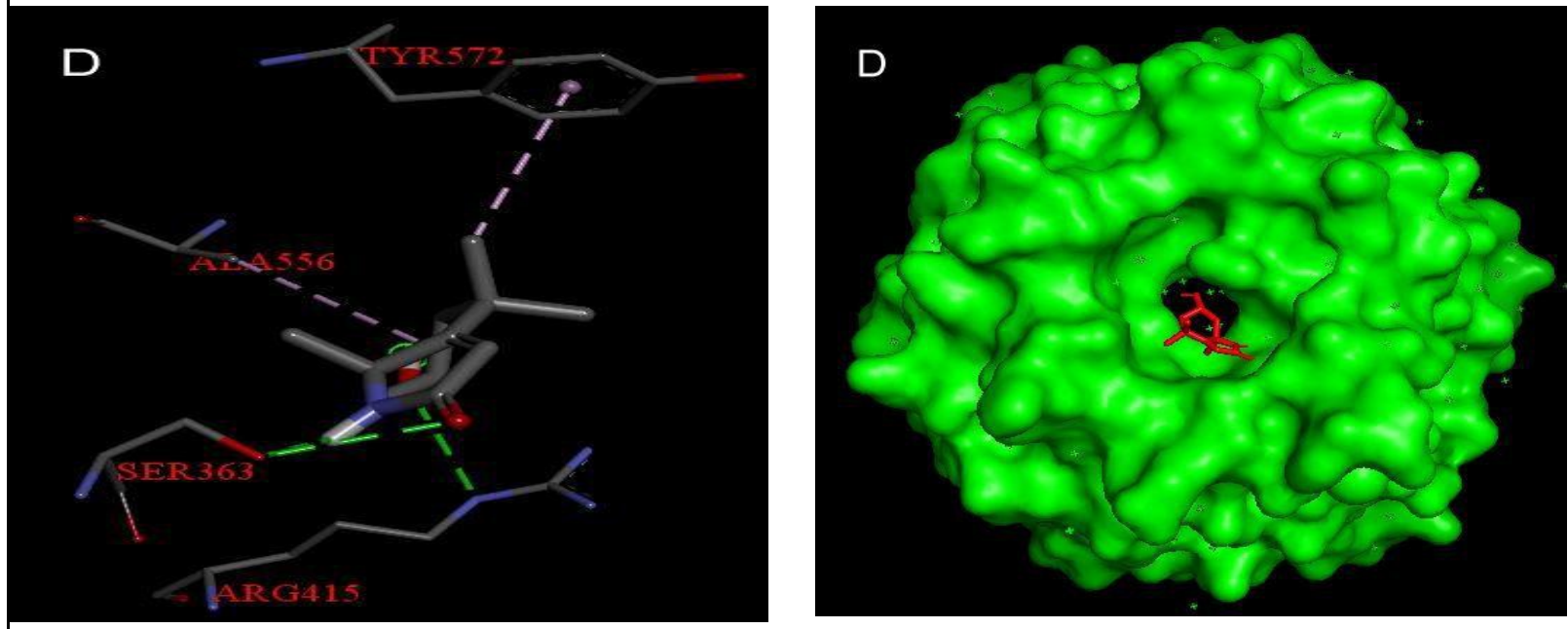

L2 

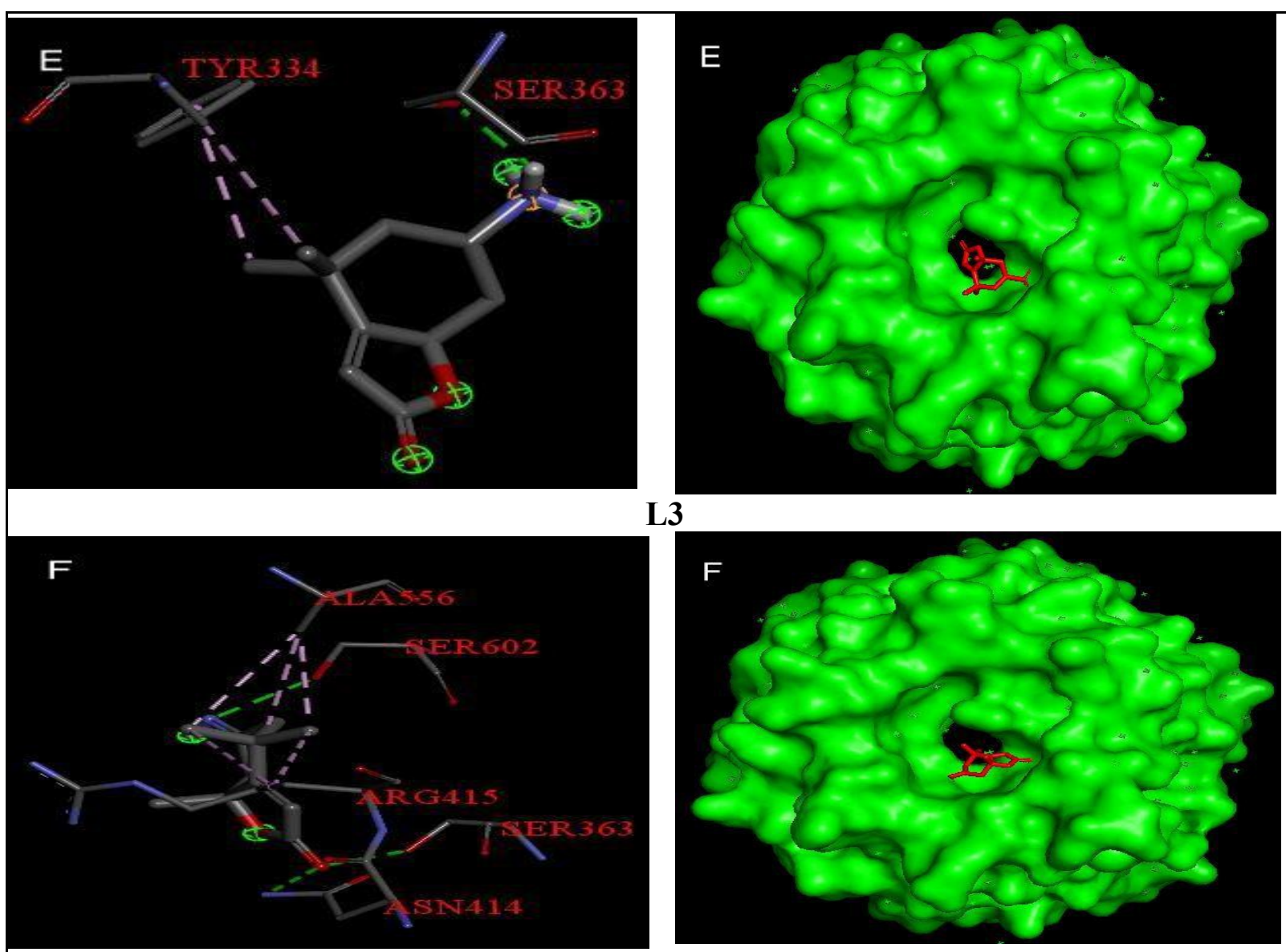

L3
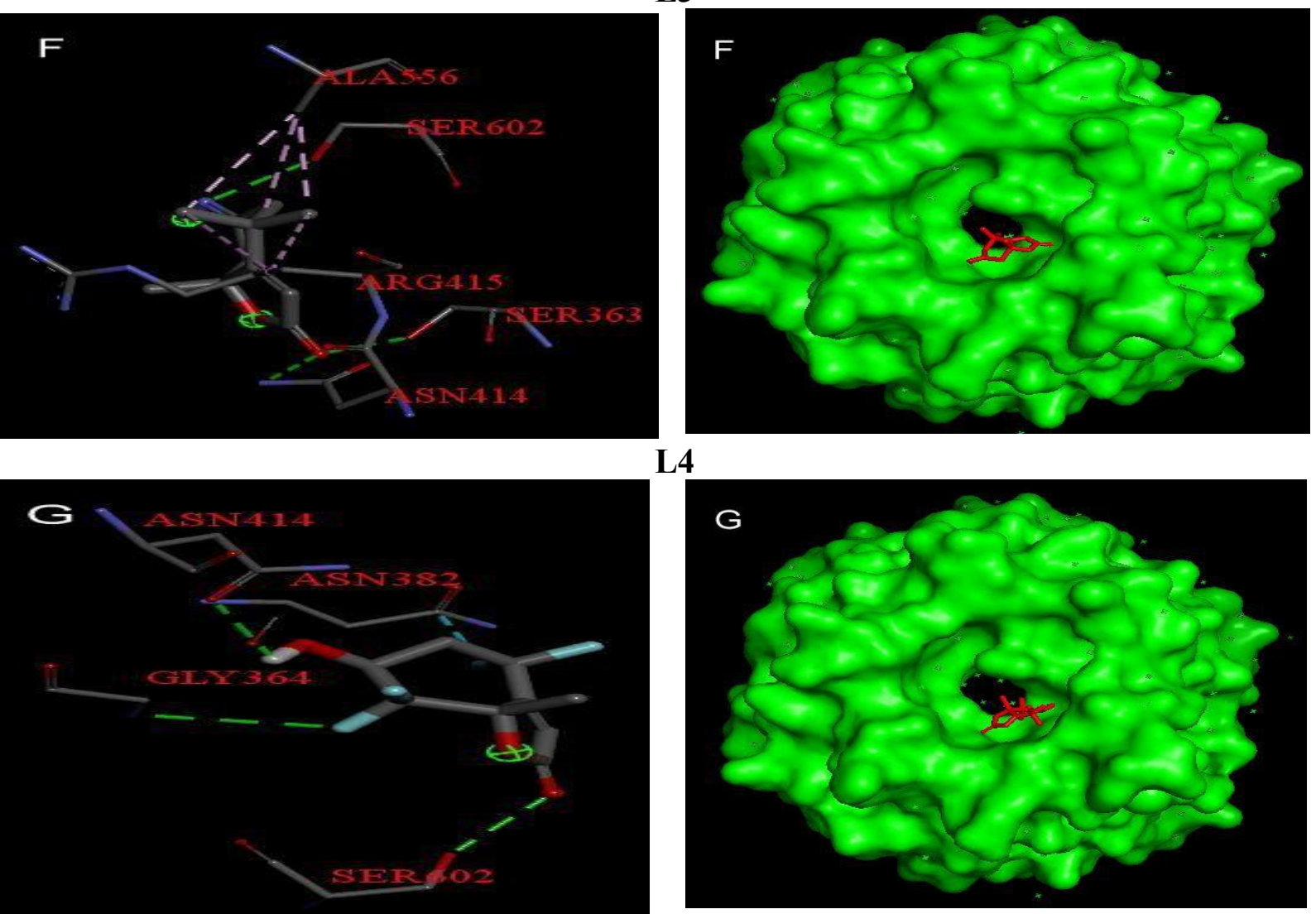

\section{L4}

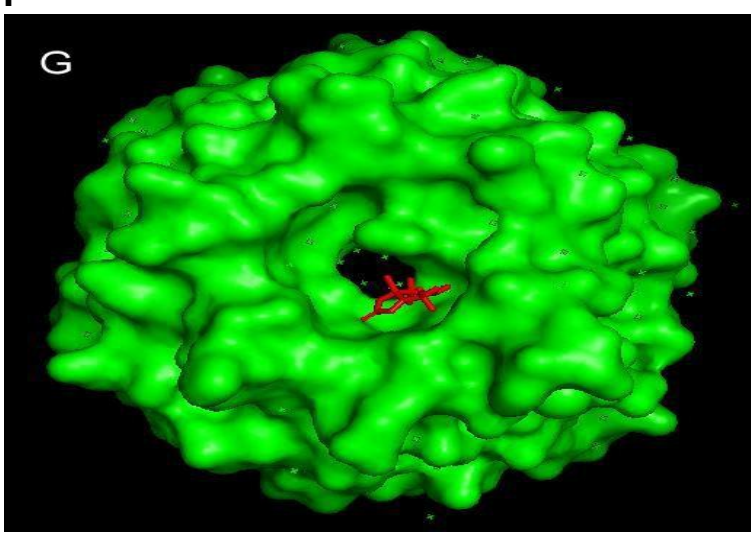




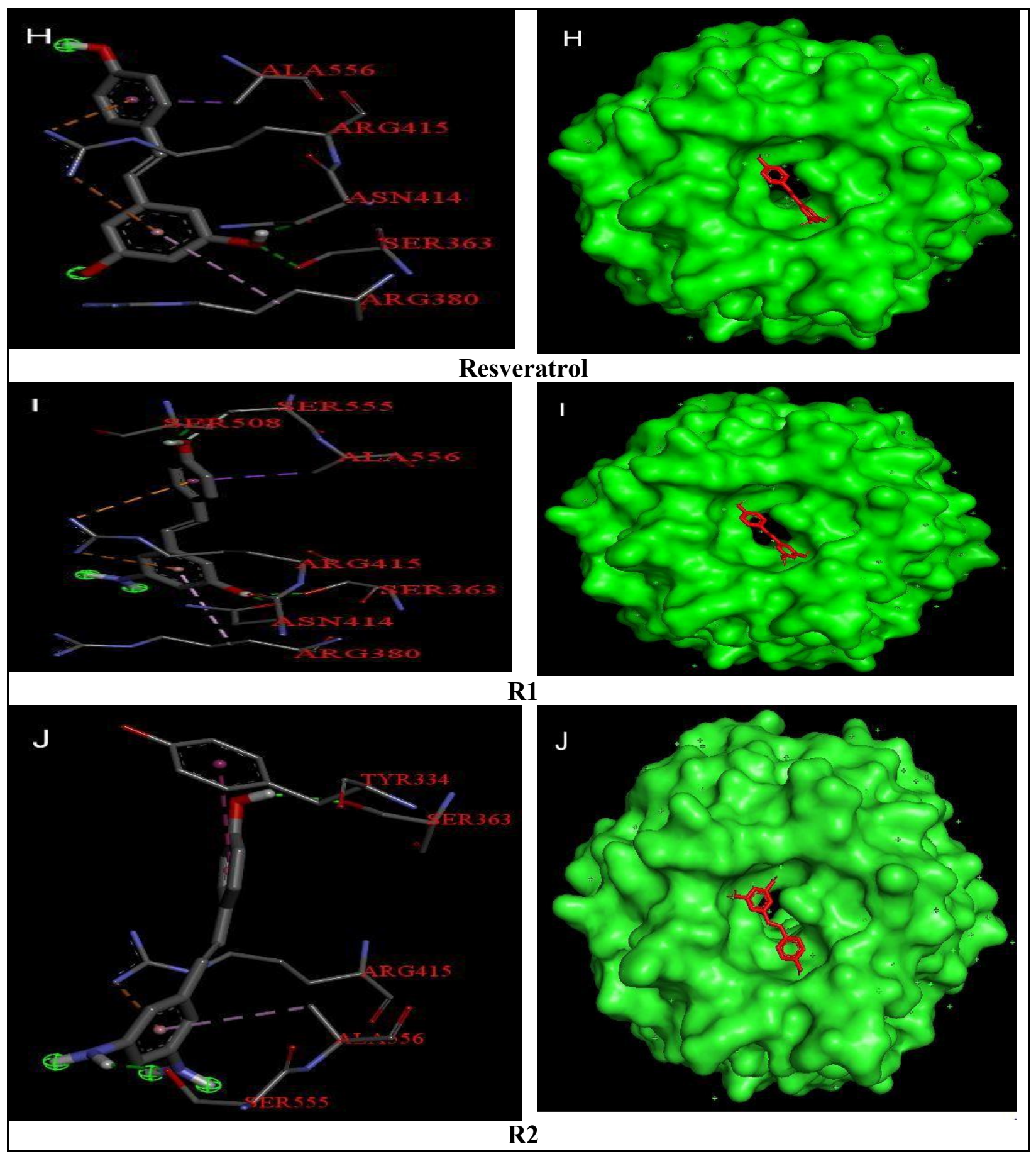




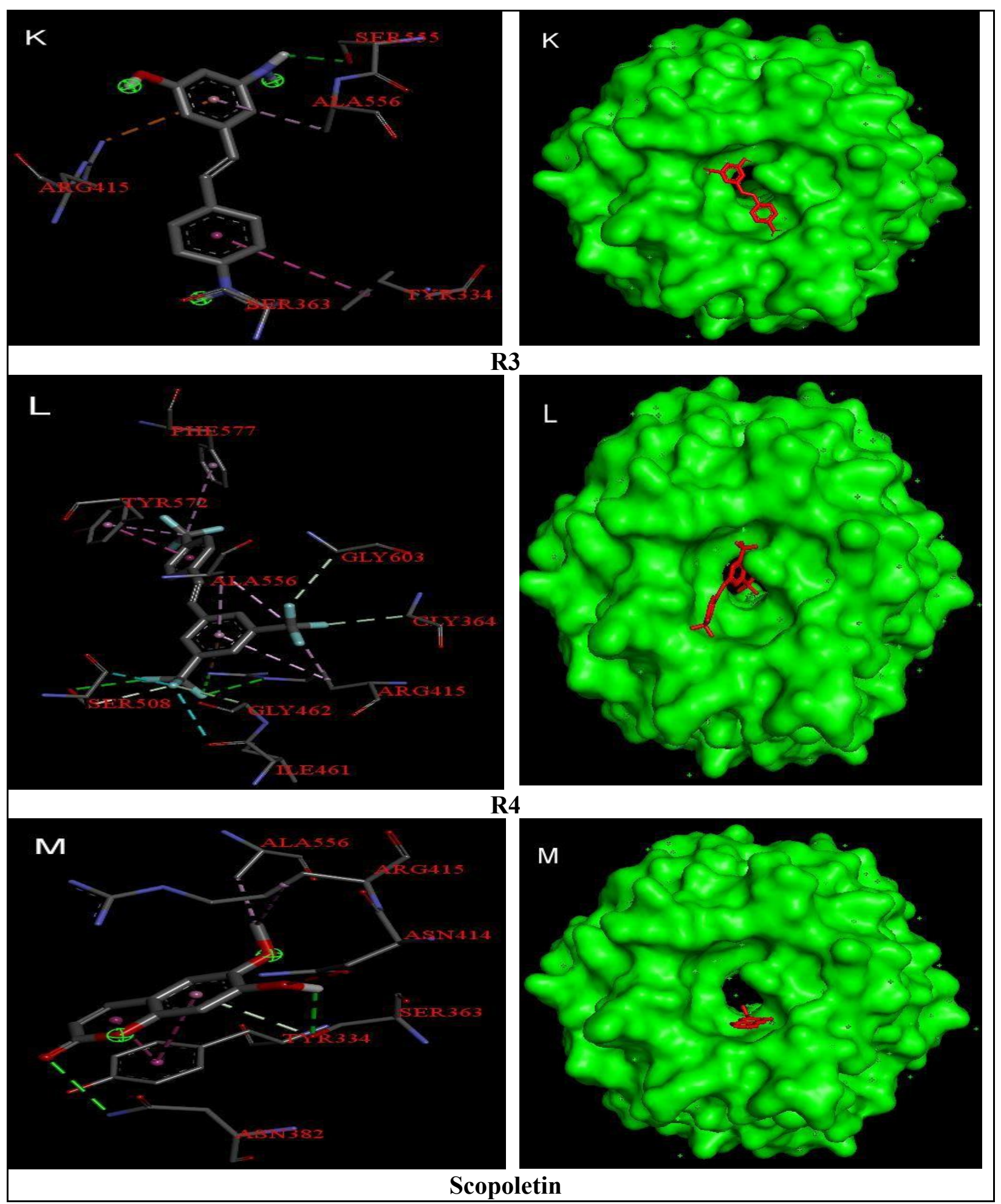




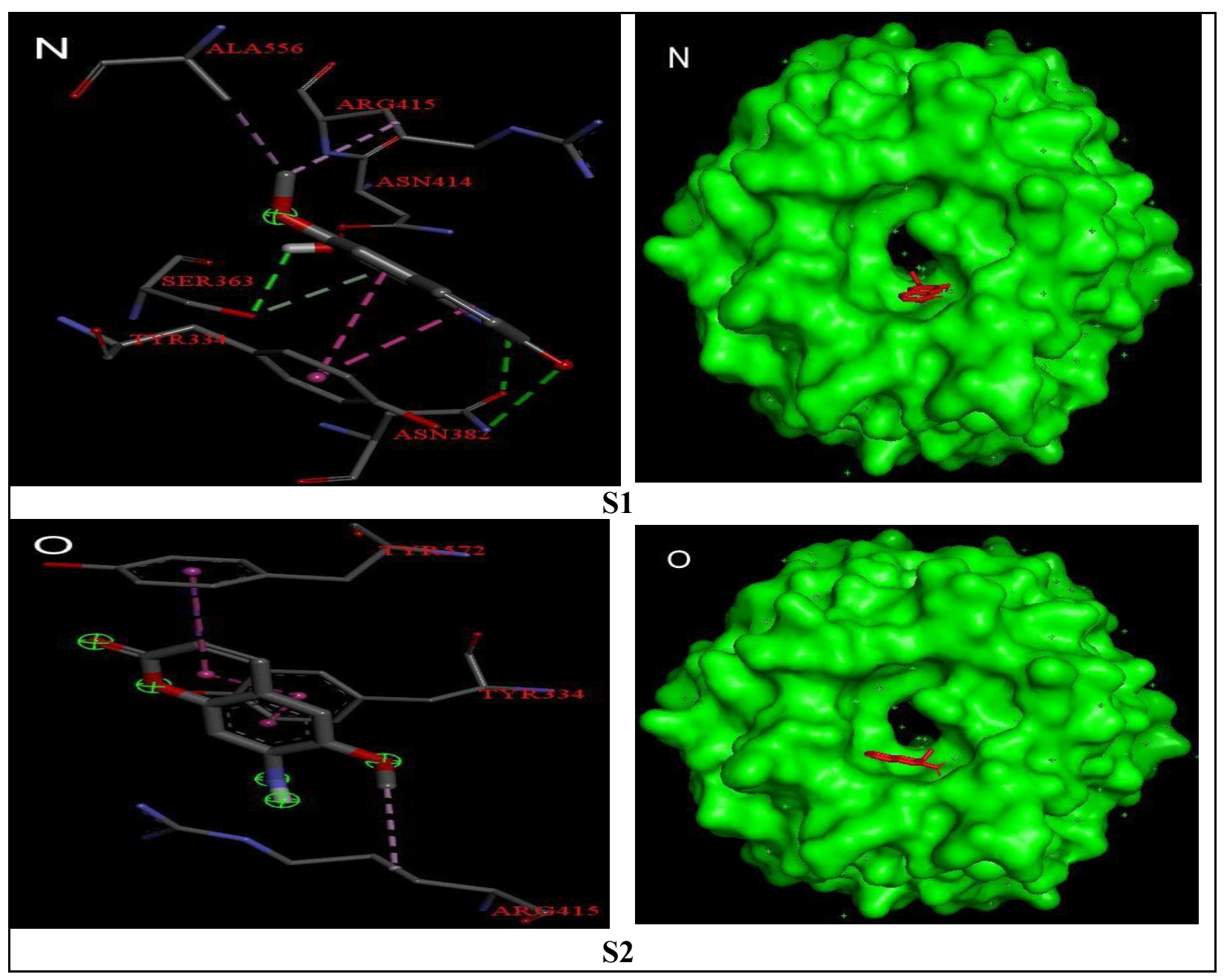




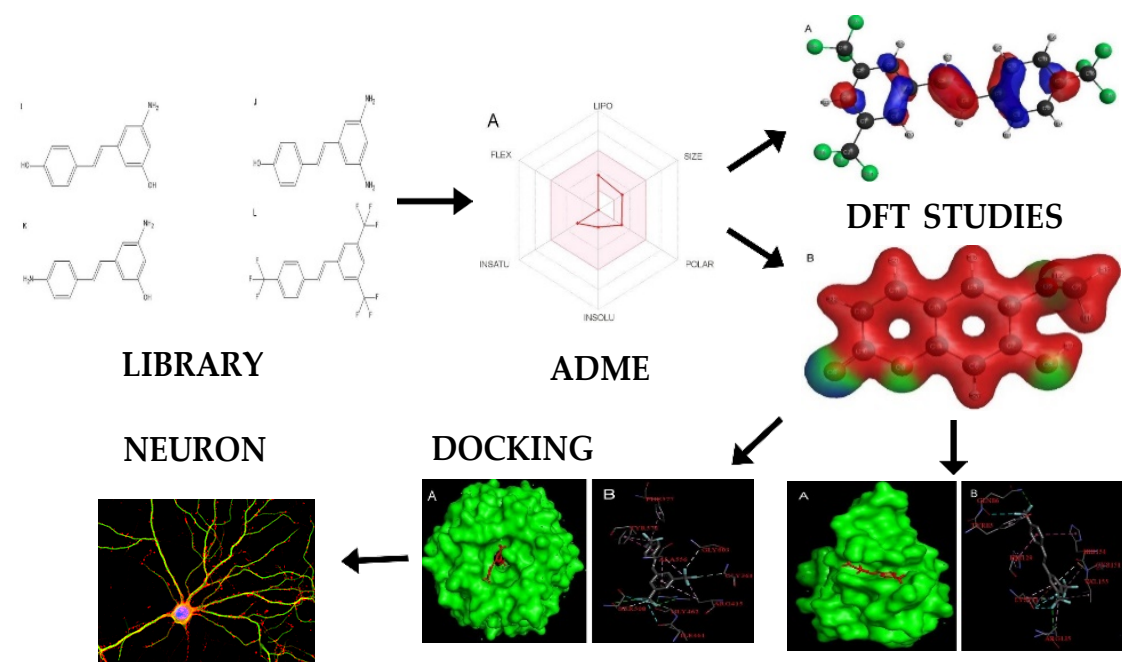

